\title{
On the locally rotationally symmetric Einstein-Maxwell perfect fluid
}

\author{
Daniela Pugliese ${ }^{1,2 *}$ and Juan A. Valiente Kroon ${ }^{1}$ \\ 1 School of Mathematical Sciences, \\ Queen Mary University of London \\ Mile End Road, London E1 4NS, UK \\ ${ }^{2}$ Institute of Physics, Faculty of Philosophy \& Science, \\ Silesian University in Opava, Bezručovo náměstí 13, \\ CZ-74601 Opava, Czech Republic
}

(Dated: September 28, 2018)

\begin{abstract}
We examine the stability of an Einstein-Maxwell perfect fluid configuration with a privileged direction of symmetry by means of a $1+1+2$-tetrad formalism. We use this formalism to cast, in a quasi linear symmetric hyperbolic form the equations describing the evolution of the system. This hyperbolic reduction is used to discuss the stability of solutions of the linear perturbation. By restricting the analysis to isotropic fluid configurations, we made use of a constant electrical conductivity coefficient for the fluid (plasma), and the nonlinear stability for the case of an infinitely conducting plasma is also considered. As a result of this analysis we provide a complete classification and characterization of various stable and unstable configurations. We found in particular that in many cases the stability conditions is strongly determined by the constitutive equations by means of the square of the velocity of sound and the electric conductivity, and a threshold for the emergence of the instability appears in both contracting and expanding systems.
\end{abstract}

PACS numbers:

Keywords: locally rotationally symmetric solutions, $1+1+2$-formalism, perturbation, stability problem, Magnetohydrodynamics

\section{INTRODUCTION}

The stability problem of plasma configurations is an important issue in a variety of astrophysical scenarios involving for example stellar objects and accretion disks, and various phenomena of the high energy Astrophysics, related to the accretion disks with the instability processes as the accretion or the Jet emission. In this article we consider the situation where gravity plays a decisive role in determining both the equilibrium states of the configurations then the dynamical phases associated to the instability, requiring a full general relativistic analysis [1-9]. Consequently these systems are described by the coupled Einstein-Maxwell-Euler equations. Notable examples are the general relativistic (GR) magnetohydrodynamic (MHD) systems. Often very complicated, the perturbation analysis must be conducted with suitable assumptions on the symmetries for the system (for example in the toroidal accretion disks) and the dynamics, numerical approaches are often required. A major challenge in dealing with these systems is to find an appropriate formulation of the problem: from one side to set up a formulation adapted to the configuration symmetries and, on the other side a suitable formulation of an initial value problem is necessary for the construction of the numerical solutions, in order to ensure the local and global existence problems and the analysis of the stability of certain reference solutions 10 for a general discussion see for example 6 , 12 , 16.

In this work we set the Einstein-Maxwell-Euler equations in a quasi-symmetric hyperbolic form, we explore the stability properties of a perfect fluid configuration with a preferred direction of symmetry coupled with the electromagnetic field. The formalism is adapted to the description of a general locally rotationally symmetric system, a remarkable example is the simple case of a spherically symmetric configurations. For a more specific discussion on the stability of spherically symmetric plasma see for example [17 [-22]. The special case of an infinitely conducting plasma describes an adiabatic flow so that the entropy per particle is conserved along the flow lines.

The plasma configuration instability, especially in the geometrically thin toroidal structures orbiting around an attractor (for example in the Shakura-Sunyaev accretion disks) is often described by the magneto-rotational instability (MRI). The dissipative (visco-resistive) effects are essential in these models as they allow the transport of angular momentum in the configurations in accretion on the central object. In fact, in the geometrically thin configurations it is assumed that the time scales of the dynamical process (balance in pressure and gravitational and centrifugal forces)

*Electronic address: d.pugliese@qmul.ac.uk

${ }^{\dagger}$ Electronic address: j.a.valiente-kroon@qmul.ac.uk 
are less then the thermal one (for dissipative heating and radiation) that is less then the viscous ones (dissipative stresses and consequent angular momentum transport). The magnetic field, the dissipative effects and the radial gradient of the plasma relativistic angular velocity are therefore essential for the MRI instability. However, some aspects of the theoretical framework of the MRI and accretion process are still to be clarified. An intriguing issue for example is the so-called visco-resistive puzzle: eventually high values of and resistivity and viscosity have to be assumed. In this work we investigate the stability problem for the systems with the magnetic field but not dissipative effects, showing the emergence of the instability and a threshold for the occurrence of the unstable modes which is essentially regulated by the conductivity parameter $\sigma_{J}$ and the speed of sound $v_{s}$, even for configurations with more specific symmetries, considered here in classes and subclasses of solutions, stable and unstable for linear perturbation. Considering both contracting and expanding systems (according to the sign of the kinematic expansion scalar $\Theta$ ), we show a threshold for the instability of the system determining two ranges for the density of matter and especially for the shear scalar $\Sigma$ along the privileged direction of the system. More specifically we consider a barotropic equation of state: when the fluid entropy is a constant of both space and time, an equation of state to link the pressure $p$ to the matter density $\rho$ can be given in the form $p=p(\rho)$ - see e.g. [10. In the present work we restrict our attention to isotropic fluids. We consider a one species particle fluid (simple fluid) and, since no particle annihilation or creation processes is expected, we use the equation of conservation of particle number. Moreover, we assume a polytropic equation of state with a constant velocity of sound and we specify the form of the conduction current using the Ohm's law, so that a linear relation between the conduction current and the electric field holds. By restricting our attention to the isotropic fluids configurations we can make use of a constant electrical conductivity coefficient for the fluid (plasma). These assumptions simplify considerably the analysis of the stability problem of linear perturbations.

A central aspect of the stability analysis to be pursued in this article is the construction of a quasi linear symmetric hyperbolic evolution system for the variables of the configuration. This, in turn, ensures the well-posed Cauchy problem for the system - in other words, the local existence and uniqueness result for the Einstein-Maxwell-Euler equations. By prescribing suitable initial data on an initial hypersurface, a unique solution exists in a neighbourhood of that hypersurface. This solution depends continuously on the initial data. Accordingly, we first write the evolution equations for the independent components of $n$ variables collected in $n$-dimensional vector $\mathbf{v}$ used to obtain a suitable symmetric hyperbolic evolution system of the form

$$
\mathbf{A}^{t} \partial_{t} \mathbf{v}-\mathbf{A}^{j} \partial_{j} \mathbf{v}=\mathbf{B v}
$$

where $\mathbf{A}^{t}$ and $\mathbf{A}^{j}$ and $\mathbf{B}$ are smooth matrix valued functions of the coordinates $(t, x)$ and the variables $\mathbf{v}$ with the index $j$ associated to some spatial coordinates $x$. The system is symmetric hyperbolic if the matrices $\mathbf{A}^{t}$ and $\mathbf{A}^{j}$ are symmetric and if $\mathbf{A}^{t}$ is a positive-definite matrix. The evolution equations is complemented by constraint and the constitutive equations. As the purpose of this work this is the study of the properties of the evolution system and the analysis of its linear stability, the problem of the propagation of the constraints will only be briefly discussed referring further details to the literature - see in particular [23, 24].

Our analysis is based on an adapted $1+1+2$-tetrad formalism for the locally rotationally symmetric spacetimes (LRS) [25, the simplest example being the spherical symmetric configurations - that is, a covariant decomposition of Einstein-Maxwell perfect fluid field equations which is particularly suitable for LRS systems. This formalism is an extension of the usual $1+3$-formalism in which the existence of a privileged timelike vector field $u^{a}$ assumed -in applications involving the description of fluids, it is natural to let $u^{a}$ to follow the congruence generated by the fluid [26]. In the $1+1+2$-formalism the presence of a further (spatial) vector field $n^{a}$ is assumed. This gives rise to a a second split of the $1+3$-reduced equations on the plane parallel and orthogonal to $n^{a}$. This type of decomposition is particularly useful in the presence of symmetries as one can naturally fix the spatial vector on the privileged symmetry direction (from now on, for simplicity "radial direction") at each point of locally rotationally symmetric spacetimes [25]. In the case of spherically symmetric spacetimes for example (here indicated with LSS), it is natural to choose $n^{a}$ to point in the radial direction of the spherical symmetry. After the decomposition, all tensors are covariantly split into scalars, vectors, and transverse-traceless 2-tensors, with respect to $n^{a}$. In the case considered here, as a result of this split, it can be shown that it is only necessary to consider radially projected tensors. Further discussion on the decomposition of Einstein-Maxwell-perfect fluid equations can be found in [25, 27, 29]. In [20] the same formalism has been used to analyse self gravitating spherically symmetric charged perfect fluid configurations in hydrostatic equilibrium. Details on the $1+3$ and $1+1+2$-decompositions of the Einstein-Maxwell equations in LRS spacetimes can be found in 30 32. We consider perturbations of the metric tensor, of the matter and EM fields, all the quantities share the same preferred direction of symmetry, as in locally rotationally symmetric (LRS) classes II space-times as described in 25] 49 .

Taking into account the gravito-electromagnetic (GEM) effects, we provide a complete classification of the solutions in terms of the scalars of the Weyl's conformal tensor. A general discussion and classification, of the solutions of the Einstein-Maxwell equations, through the scalars of the Weyl tensor can be found in 39 .

Here the consider the electromagnetic fields considered through real vector functions, while in many of the LSR 
spacetimes in [27, 29, 30, 33, 34, was naturally used the complex variable $\psi=E+i B$ and $\psi^{*}=E-i B$ in order to decouple the equations with the appropriate symmetries and obtain linear equations in the fields. As pointed out in 27] there are two ways to proceed depending of how one considers the coupled fields and test fields: specifically on how one views the Maxwell's and Einstein's equations, and the gravitational effects on the EM field, in other words the analysis of test fields on a background. The gravitational background must, in any case, have the same symmetries of LRS systems: a suitable example is therefore the spherically symmetric schwarzschild solution. Alternatively, one can consider other scenarios, we refer to a general discussion of the analysis in [27, 29, 30, 33, 34]. Here we simply observe that such a system can be self-gravitating or not; one can consider the perturbations of the background or otherwise to fix the spacetime.

In this article we address the more general case, considering also the perturbations of the gravitational part, in terms of scalars of the Weyl tensor. We assumed the symmetries to be preserved by the perturbation and limiting the analysis to constant velocity of sound and conductivity parameter. To simplify the discussion of the results, the calculation procedures, and the complete systems before the assumption of one privileged symmetry of direction, are specified in some Appendix Sections.

The procedure of hyperbolic reduction used in the present article follows the presentation given in 40. This particular analysis is independent of geometric gauge considerations [50. In the present work we also provide a suitable propagation equation for the fluid radial acceleration. To this end, we introduce an auxiliary field corresponding to the derivative of the matter density projected along the radial direction. suitable field and evolution equations can be obtained for this quantity. The resulting evolution system is then used to analyse the stability problem for small nonlinear perturbations of a background solution. More precisely, we perform a first order perturbation to $\mathbf{v}$ of the form $\mathbf{v} \mapsto \epsilon \dot{\mathbf{v}}+\breve{\mathbf{v}}$, where the parameter $\epsilon$ sets the order of the perturbation while $\breve{\mathbf{v}}$ describes the (linear) perturbation of the background solution. Now, assuming the background variables $\stackrel{\mathrm{v}}{\mathrm{v}}$ to satisfy the evolutions we end up with an evolution system for the perturbations of the form

$$
\stackrel{̊}{\mathbf{A}}^{t} \partial_{t} \breve{\mathbf{v}}-\stackrel{\AA}{\mathbf{A}}^{j} \partial_{j} \breve{\mathbf{v}}=\stackrel{\circ}{\mathbf{B}} \breve{\mathbf{v}} .
$$

The core of the stability analysis consists of the study of the term $\mathbf{B}$ using some relaxed stability eigenvalue conditions. The procedure to analyse stability used here is adapted from 24] - see also discussion in [42] and cited references. Under suitable circumstances it can be regarded as a first step toward de analysis of non-linear stability. In our case, the elements of matrix $\stackrel{\circ}{\mathbf{B}}$ are, in general, functions of the space and time coordinates. For a general discussion on the time dependent case and the case of non constant matrix coefficient (depending on both time and space) we refer to 24]. The case of a linearised system where the coefficients are constant matrices is discussed in [45]. Finally, the case of systems with vanishing eigenvalues has been discussed in [46 48. In our case, a fully analysis of the stability properties of the system turns out extremely cumbersome because of the form of $\mathbf{B}^{\circ}$ associated to the present problem. We will proceed with the analysis of the values of the eigenvalues using indirect methods based on the inspection of the characteristic polynomial. In order to keep the problem manageable, we analyse a number of simplified systems obtained by making some assumptions about the configuration. More precisely, we consider background configurations with a vanishing radial acceleration case for the reference solution, and we explore particular models with fixed values of the kinematic scalars. This analysis constitutes the main result of the article. We provide a detailed classification by considering systems with particular kinematic configurations defined by fixing the radial acceleration of the four velocity, and the $1+1+2$-projected expansion, shear, twisting and the vorticity of the system: the stability conditions can be strongly determined by the constitutive equations by means of the square of the velocity of sound and the electric conductivity.

Since a significant part of this work was dedicated to the formalization of the problem in symmetric hyperbolic form, a first part of the article was necessarily devoted to the presentation of the formalism and the explanation of the adopted notation. To simplify the discussion of the problem and the illustration of the results the article has been developed into three parts: in the first (I) part, from Sec. (II) to Sec. (V), we introduced the $1+1+2$ formalism decomposing the equations and set system in symmetric hyperbolic form, which is the first outcome of this work. The second (II) part, Sec. (VI) and Sec. (VII), develops the perturbations and the system stability is analysed. Part II constitutes the main part of this article with a major discussion of the main results on the system stability in the fundamental classes of the solutions. A more specific discussion on the other subclasses can be found in the final part of this article. The third (III) part is constituted by the Appendix Sections, deepening details of the I and II parts, and explaining some important aspects of the decomposition procedure. We show the perturbed equations in symmetric hyperbolic form for the general case of non-zero radial acceleration discussing in detail the conditions for the unstable configurations belonging to the various classes and subclasses of solutions.

In details, the present article is structured as follows: the $1+3$-formalism is briefly reviewed in Section IIA. The $1+1+2$-decomposition that will be used in our present analysis is discussed in Section IIB. In Section III] we write the $1+1+2$-equations for the LRS system. Section[IV]provides a discussion on the thermodynamical quantities of the 
system. A summary of the evolution equations is given in Section $\mathrm{V}$. The re-parametrised set of evolution equations considered for the stability analysis is given in Section VA. Section VI discusses the perturbation to the first order of the variables. Section VIA provides some general remarks on the set of perturbed equations and system stability. Section VII contains the main results concerning the nonlinear stability of the symmetric hyperbolic system. Some concluding remarks are given in Section VIII. Finally, in Appendix A we provide an alternative symmetric hyperbolic system for the fluid variables. The $1+1+2$-decomposition of these equations is in Appendix B, Some general notes on the evolution equations and hyperbolicity considerations can be found in Appendix C

\section{PRELIMINARIES}

We consider the stability problem for a configuration with a spatial direction of symmetry (privileged direction of the system admitting a one-dimensional isotropy group), described by the Einstein-Maxwell-Euler equations for a perfect fluid. By applying the $1+1+2$ decomposition i we can take full advantage of the symmetries of the LRS system, and moreover this procedure allows to construct in an easy, and relatively immediate manner, a quasi-linear hyperbolic system. This ensures also the consistency of possible numerical approaches to the problems, without requiring the introduction of any auxiliary variable to handle both the propagation equations along the timelike direction then the constraint part of the system. Morover, the covariant and gauge-invariant perturbation formalism turn to be especially suitable for dealing with spacetimes with some preferred spatial direction, not necessary spherical symmetry in the background, and to the application possibly to the case of gravitational wave propagation by introducing a radial unit vector, decomposing all covariant quantities with respect to this [13, 25, 27, 28, All the equations and the quantities related to the fields and the curvature tensor will be decomposed according to the $1+1+2$ procedure. For this purpose it will be necessary to first fix the notation induced by the $1+3$-decomposition as introduced Sec. (II A). The procedure of $1+1+2$-decomposition, adapted to the symmetries of the system, will be discussed with some details in Sec. (II B).

\section{A. The $1+3$-formalism}

The implementation of the $1+3$-formalism used in the present article follows, as much as possible, the notation and conventions of [26]. We consider 4-dimensional metrics $g_{a b}$ with signature $(-,+,+,+)$. The Latin indices $a, b, c \ldots$ will denote spacetime tensorial indices taking the values $(0,1,2,3)$ while $i, j, k \ldots$ will correspond to spatial frame indices ranging over $(1,2,3)$. The Levi-Civita covariant derivative of $g_{a b}$ will be denoted by $\nabla_{a}$. Whenever convenient, we use the semicolon notation. As usual, one has that $\nabla_{a} g_{b c}=g_{b c ; a}=0$.

In what follows, the timelike vector field (flow vector) $u^{a}$ will describe the normalised future directed 4-velocity of the fluid. It satisfies $u^{a} u_{a}=-1$. Indices are raised and lowered with $g_{a b}$. The tensor $h^{a b} \equiv g^{a b}+u^{a} u^{b}$ is the projector onto the three dimensional subspace orthogonal to $u^{a}$, thus, one has that

$$
h_{b}^{a}=\delta^{a}{ }_{b}+u^{a} u_{b}, \quad h^{b}{ }_{a} h^{a}{ }_{c}=h^{b}{ }_{c}, \quad h^{a}{ }_{b} h_{a}^{b}=3, \quad h^{a}{ }_{b} u_{a}=0 .
$$

Following the standard approach of $1+3$-formalisms, we split the first covariant derivative of $u^{b}$ as

$$
\nabla_{a} u_{b}=\sigma_{a b}+\omega_{a b}+\frac{1}{3} \Theta h_{a b}-\dot{u}_{b} u_{a}
$$

where $\sigma_{a b} \equiv D_{\langle b} u_{a\rangle}$ with $\sigma_{a b} u^{b}=0, \omega_{a b} \equiv D_{[a} u_{b]}$ with $\omega_{a b} u^{b}=0, \Theta \equiv D^{a} u_{a}$ and $\dot{u}_{a}=u^{b} \nabla_{b} u_{a}$ are, respectively, the shear and the vorticity tensors, the volume expansion scalar, and the 4 -acceleration vector. Moreover, we introduce the vorticity vector $\omega^{a} \equiv \epsilon^{a b c} \omega_{b c} / 2$ where $\epsilon_{a b c}=u^{d} \epsilon_{d a b c}, \epsilon_{a b e} \epsilon^{a b e}=6$ and $\epsilon_{d a b c}$ stands for the totally antisymmetric tensor with $\epsilon_{0123}=\sqrt{-\operatorname{det} g_{a b}}$. Then, $\sigma_{a b} u^{a}=0=\omega_{a b} u^{a}=\dot{u}_{a} u^{a}$ by construction. In the above expressions, the operator $D_{a}$ corresponds to the 3-dimensional covariant derivative obtained from projecting the spacetime covariant derivative in the distribution orthogonal to $u_{b}$. As an example of this procedure, for a generic 2-rank tensor $T_{b c}$, one has that $D_{a} T_{b c}=h^{s}{ }_{a} h^{t}{ }_{b} h^{p}{ }_{c} T_{s t ; p}$, and $T_{s t ; p}=\nabla_{p} T_{s t}$ while $\dot{T}_{s t} \equiv u^{a} \nabla_{a} T_{s t}$. For clarity, whenever necessary, projected indices of a tensor will be highlighted by \langle\rangle - brackets. For example, we write $T_{\langle a b\rangle}=h_{a}{ }^{c} h_{b}{ }^{d} T_{c d}$.

\section{B. The $1+1+2$-formalism}

The implementation of a $1+1+2$-formalism to be used in the present article follows the notation and conventions of [25]. In what follows, let $n_{a}$ denote a spacelike normalised vector $n_{a}$ chosen along a preferred direction of the 
spacetime and which define a further projector tensor $N_{a}^{b}$

$$
n_{a} n^{a}=1, \quad u_{a} n^{a}=0, \quad N_{a}^{b} \equiv h_{a}^{b}-n_{a} n^{b}=\delta_{a}^{b}+u_{a} u^{b}-n_{a} n^{b}, \quad N_{a}^{b} u^{a}=N_{a}^{b} n^{a}=0,
$$

on the space orthogonal to both $n_{a}$ and $u_{a}$-a 2-dimensional sheet. In addition to this projector, we also introduce the following rank 2 totally antisymmetric tensor

$$
\begin{gathered}
\epsilon_{a b} \equiv \epsilon_{a b c} n^{c}=\epsilon_{d a b c} u^{d} n^{c}, \quad \epsilon_{(a b)}=\epsilon_{a b} n^{b}=0, \quad \epsilon_{a b c}=n_{a} \epsilon_{b c}-n_{b} \epsilon_{a c}+n_{c} \epsilon_{a b}, \\
\epsilon_{a b} \epsilon^{c d}=N_{a}^{c} N_{b}^{d}-N_{a}^{d} N_{b}^{c}, \quad \epsilon_{a}^{c} \epsilon_{b c}=N_{a b}, \quad \epsilon^{a b} \epsilon_{a b}=2 .
\end{gathered}
$$

Given 3-vector $\psi^{a}$, one can use the projector $N_{a}^{b}$ to split it as $\psi^{a}=\Psi n^{a}+\Psi^{\bar{a}}$, with $\psi^{\bar{a}} \equiv \psi_{b} N^{a b}$, lying in the sheet orthogonal to $n_{a}$. In order to avoid any confusion, we make use of an overbar on index to denote the projection with $N_{a}^{b}$. By means of the projector $N_{a}^{b}$ one can decompose the kinematical quantities $\dot{u}_{a}$ and $\omega_{a}$ in the form $\dot{u}^{a}=\mathcal{A} n^{a}+\mathcal{A}^{a}, \quad \omega^{a}=\Omega n^{a}+\Omega^{a}$, with $\mathcal{A} \equiv n^{a} u^{b} \nabla_{b} u_{a}$ and $\Omega$ describing the components of the acceleration and the vorticity in the direction of $n^{a}$. Now, any spatially projected, symmetric, trace-free (PSTF) tensor $\psi_{a b}$ can be split into a scalar $\Psi=\psi^{b} n_{b}$, a 2 -tensor $\Psi_{a b}$ and a vector $\Psi_{a}$

$$
\psi_{a b}=\psi_{\langle a b\rangle}=\Psi\left(n_{a} n_{b}-\frac{1}{2} N_{a b}\right)+2 \Psi_{(a} n_{b)}+\Psi_{a b},
$$

where

$$
\Psi \equiv \psi_{a b} n^{a} n^{b}=-N^{a b} \psi_{a b}, \quad \Psi_{a} \equiv N_{a}^{b} n^{c} \psi_{b c}=\psi_{\bar{a}}, \quad \Psi_{a b} \equiv \psi_{\{a b\}} \equiv\left(N_{(a}^{c} N_{b)}^{d}-\frac{1}{2} N_{a b} N^{c d}\right) \phi_{c d}
$$

In the above expressions, the curly brackets indicate the transverse-traceless part of the corresponding tensor. Note that in the present context,the term "transverse" only refers to the fact that the tensor is orthogonal to $n_{a}$. In the following, where useful, we will denote with $\left(^{\perp}\right)$ the component of any tensor orthogonal to $n_{a}$ and with $(")$ the component parallel to it. It is convenient to further define the following two derivatives:

$$
\hat{\phi}_{a \cdots b}{ }^{c \cdots d} \equiv n^{e} D_{e} \phi_{a \cdots b}{ }^{c \cdots d}, \quad \delta_{e} \phi_{a \cdots b}{ }^{c \cdots d} \equiv N_{e}^{j} N_{a}^{f} \cdots N_{b}^{g} N_{h}^{c} \cdots N_{i}^{d} D_{j} \phi_{f \cdots g}{ }^{h \cdots i}
$$

Accordingly, one can write

$$
D_{a} n_{b}=n_{a} a_{b}+\frac{1}{2} \Phi N_{a b}+\xi \epsilon_{a b}+\zeta_{a b}, \quad a_{a} \equiv n^{c} D_{c} n_{a}=\hat{n}_{a}, \quad \Phi \equiv \delta_{a} n^{a}, \quad \xi \equiv \frac{1}{2} \epsilon^{a b} \delta_{a} n_{b}, \quad \zeta_{a b} \equiv \delta_{\{a} n_{b\}} .
$$

The scalar $\Phi$ denotes is the expansion of the 2-sheet generated by $n_{a}, \zeta_{a b}$ the shear of $n^{a}$ and $\xi$ is the rotation of $n^{a}$ (i.e. the twisting of the 2 - sheet), finally $a^{a}$ is the acceleration. Finally, also define the derivative

$$
\dot{n}_{a}=\mathcal{A} u_{a}+\alpha_{a}, \quad \alpha_{a} \equiv \dot{n}_{\bar{a}}
$$

It can then be verified that

$$
\dot{N}_{a b}=2 u_{(a} \dot{u}_{b)}-2 n_{(a} \dot{n}_{b)}=2 u_{(a} \mathcal{A}_{b)}-2 n_{(a} \alpha_{b)}, \quad \hat{N}_{a b}=-2 n_{(a} a_{b)}, \quad \delta_{c} N_{a b}=0
$$

and, furthermore, that

$$
\dot{\epsilon}_{a b}=-2 u_{[a} \epsilon_{b] c} \mathcal{A}^{c}+2 n_{[a} \epsilon_{b] c} \alpha^{c}, \quad \hat{\epsilon}_{a b}=2 n_{[a} \epsilon_{b] c} a^{c}, \quad \delta_{c} \epsilon_{a b}=0 .
$$

On the decomposition of the kinematic quantities Applying the decomposition (6) to the symmetric trace free tensor $\sigma_{a b}$ appearing in the $1+3$-decomposition given by equation $(2)$ one obtains

$$
\sigma_{a b}=\Sigma\left(n_{a} n_{b}-\frac{1}{2} N_{a b}\right)+2 \Sigma_{(a} n_{b)}+\Sigma_{a b}
$$

where we have introduced the scalar $\Sigma$ (the totally projected part of the shear of the 3-sheet), the vector $\Sigma_{a}$ and the 2-tensor $\Sigma_{a b}$ and defined as in equations (7).

The full covariant derivative of $n^{a}$ and $u^{a}$ can now be written as

$$
\begin{aligned}
& \nabla_{a} n_{b}=-\mathcal{A} u_{a} u_{b}-u_{a} \alpha_{b}+\left(\Sigma+\frac{1}{3} \Theta\right) n_{a} u_{b}+\left(\Sigma_{a}-\epsilon_{a c} \Omega^{c}\right) u_{b}+n_{a} a_{b}+\frac{1}{2} \phi N_{a b}+\xi \epsilon_{a b}+\zeta_{a b} \\
& \nabla_{a} u_{b}=-u_{a}\left(\mathcal{A} n_{b}+\mathcal{A}_{b}\right)+n_{a} n_{b}\left(\frac{1}{3} \Theta+\Sigma\right)+n_{a}\left(\Sigma_{b}+\epsilon_{b c} \Omega^{c}\right)+\left(\Sigma_{a}-\epsilon_{a c} \Omega^{c}\right) n_{b}+N_{a b}\left(\frac{1}{3} \Theta-\frac{1}{2} \Sigma\right)+\Omega \epsilon_{a b}+\Sigma_{a b}
\end{aligned}
$$


The decomposition of the Weyl tensor Let $C_{a b c d}$ denote the Weyl curvature tensor of the metric $g_{a b}$. As it is well known, it can be $1+3$-decomposed in terms of symmetric, traceless 2-rank tensors defined by

$$
\begin{aligned}
& \mathcal{E}_{a b} \equiv C_{a b c d} u^{c} u^{d}=\mathcal{E}\left(n_{a} n_{b}-\frac{1}{2} N_{a b}\right)+2 \mathcal{E}_{(a} n_{b)}+\mathcal{E}_{a b}, \\
& \mathcal{B}_{a b} \equiv \frac{1}{2} \epsilon_{a d e} C_{b c}^{d e} u^{c}=\mathcal{B}\left(n_{a} n_{b}-\frac{1}{2} N_{a b}\right)+2 \mathcal{B}_{(a} n_{b)}+\mathcal{B}_{a b},
\end{aligned}
$$

- the so-called electric $\mathcal{E}_{a b}$ and magnetic $\mathcal{B}_{a b}$ parts with respect to $u^{a}$. Using equation [6], these rank 2 tensors are $1+1+2$-decomposed where the scalars $\mathcal{E}$ and $\mathcal{B}$ denote, respectively, the totally projected electric and magnetic parts of the Weyl tensor.

Decomposition of the matter fields The energy momentum tensor $T_{a b}$ describing the matter-field content of the system under consideration is of the form

$$
T_{a b}=T_{a b}^{\mathrm{f}}+T_{a b}^{\mathrm{em}}, \quad T_{a b}^{\mathrm{f}}=\rho u_{a} u_{b}+p h_{a b},
$$

where $T_{a b}^{\mathrm{f}}$ denotes the energy-momentum tensor of the perfect fluid given by the well-known expression with $\rho$ and $p$ denoting, respectively, the total energy density and pressure as measured by an observer moving with the fluid. Accordingly, the timelike vector field (flow vector) $u^{a}$ denotes the normalised future directed 4-velocity of the fluid. The electromagnetic energy momentum tensor $T_{a b}^{\mathrm{em}}$ is given by

$$
T_{a b}^{\mathrm{em}}=\left(F_{a c} F_{b}^{c}-\frac{1}{4} F_{c d} F^{c d} g_{a b}\right), \quad F_{a b}=-\left(2 E_{[a} u_{b]}-\epsilon_{a b c} B^{c}\right) .
$$

where $F_{a b}$ is the electromagnetic field (Faraday) tensor, the Faraday tensor has been split in its electric part, $E_{a} \equiv F_{a b} u^{b}$, and its magnetic part, $B^{a} \equiv \frac{1}{2} \epsilon^{a b c} F_{c d}$, with respect to the flow. Alternatively, one can rewrite equation 18 in the form

$$
T_{a b}^{\mathrm{em}}=\frac{1}{2} u_{a} u_{b}\left(E^{2}+B^{2}\right)+\frac{1}{6} h_{a b}\left(E^{2}+B^{2}\right)+P_{a b}+2 \mathcal{G}_{(a} u_{b)},
$$

where we have written $E^{2} \equiv E_{a} E^{a}$ and $B^{2} \equiv B^{a} B_{a}$, and $P_{a b}$ denotes the symmetric, trace-free tensor given by

$$
P_{a b}=P_{(a b)} \equiv \frac{1}{3} h_{a b}\left(E^{2}+B^{2}\right)-\left(E_{a} E_{b}+B_{a} B_{b}\right) .
$$

Furthermore, $\mathcal{G}_{a} \equiv \epsilon_{a u v} E^{u} B^{v}$, denotes the Poynting vector. Thus we obtain

$$
T_{a b}^{\mathrm{em}} n^{a} n^{b}=\frac{1}{2}\left(E^{2}+B^{2}\right)-\left({ }^{\|} E^{2}+{ }^{\|} B^{2}\right), \quad E^{a}={ }^{\|} E n^{a}+{ }^{\perp} E^{a}, \quad B^{a}={ }^{\|} B n^{a}+{ }^{\perp} B^{a}
$$

the electric $E^{a}$ and magnetic $B^{a}$ are split relative to the vector $n^{a}$. Now, recall that he Maxwell equations can be written as

$$
\nabla_{b} F^{a b}=J^{a}, \quad \nabla_{[a} F_{b c]}=0 \quad \text { where } \quad J_{a}=\varrho_{C} u_{a}+{ }^{(3)} j_{a}, \quad{ }^{(3)} j^{a}={ }^{\|} j n^{a}+{ }^{\perp} j^{a},
$$

where $J^{a}$ is the 4 -current. where $\varrho_{C}$ is the charge density, and the 3 -current is ${ }^{(3)} j_{a}$.

\section{III. $1+1+2$-EQUATIONS FOR LRS SYSTEM}

Evolution equations adapted to the $1+1+2$-decomposition of spacetime discussed in the previous Sections can be readily obtained from the $1+3$-evolution equations using the split of equation (6) for a symmetric, trace-free tensor and projecting the original propagation equations along the longitudinal and orthogonal directions given by the vector $n^{a}$. For a generic symmetric, trace-free tensor one obtains three equations for the projected components $\Psi, \Psi_{a}$ and $\Psi_{a b}$. In the case of a LRS and LSS systems it is only necessary to consider the evolution equation of the radially projected component - i.e. the scalar $\Phi$. This approach is allowed in any spacetimes with a preferred spatial direction at each point, the so-called locally rotationally symmetric spacetimes (LRS) - see e.g. [25].

In the case LSS spacetimes, $n^{a}$ is a vector pointing along the axis of symmetry and can therefore be thought of as being a radial vector. As there is no preferred directions in the 2-surface sheet all the non-radial components of the various tensors are assumed to vanish. Consequently, the kinematical quantities can be decomposed as

$$
\dot{u}^{a}=\mathcal{A} n^{a}, \quad \omega^{a}=\Omega n^{a}, \quad \sigma_{a b}=\Sigma\left(n_{a} n_{b}-\frac{1}{2} N_{a b}\right) .
$$


so that

$$
\begin{aligned}
& n_{a b} \equiv \nabla_{a} n_{b}=-\mathcal{A} u_{a} u_{b}+\left(\Sigma+\frac{1}{3} \Theta\right) n_{a} u_{b}+\frac{1}{2} \Phi N_{a b}+\xi \epsilon_{a b}, \\
& u_{a b} \equiv \nabla_{a} u_{b}=-\mathcal{A} u_{a} n_{b}+n_{a} n_{b}\left(\Sigma+\frac{1}{3} \Theta\right)+N_{a b}\left(\frac{1}{3} \Theta-\frac{1}{2} \Sigma\right)+\Omega \epsilon_{a b},
\end{aligned}
$$

while the decomposition of the Weyl tensor reduces to $\mathcal{E}_{a b}=\left\|\mathcal{E}\left(n_{a} n_{b}-\frac{1}{2} N_{a b}\right), \quad \mathcal{B}_{a b}=\right\| \mathcal{B}\left(n_{a} n_{b}-\frac{1}{2} N_{a b}\right)$. In an analogous manner, it can be seen that the only non-vanishing components for the matter fields are given by the scalars $\left(\rho, p,{ }^{\|} E,{ }^{\|} B, \varrho_{C},{ }^{\|} j\right)$.

Evolution equation for the Maxwell fields Starting from the usual 1+3-decomposition of the Maxwell equations - see for example [26] - and applying the general procedure described at the beginning of the previous Section one readily obtains the following $1+1+2$-evolution equations

$$
\begin{aligned}
& { }^{\|} \dot{E}=2 \xi^{\|} B-\left(\frac{2}{3} \Theta-\Sigma\right){ }^{\|} E+{ }^{\perp} E^{a}\left(\alpha_{a}+\Sigma_{a}\right)+\epsilon_{a b} \delta^{a \perp} B^{b}+\epsilon_{a b}\left(\mathcal{A}^{a \perp} B^{b}+\Omega^{a \perp} E^{b}\right)-{ }^{\|} j, \\
& { }^{\|} \dot{B}=-2 \xi^{\|} E-\left(\frac{2}{3} \Theta-\Sigma\right){ }^{\|} B-\epsilon_{a b} \delta^{a \perp} E^{b}+{ }^{\perp} B^{a}\left(\alpha_{a}+\Sigma_{a}\right)-\epsilon_{a b}\left(\mathcal{A}^{a \perp} E^{b}-\Omega^{a \perp} B^{b}\right) .
\end{aligned}
$$

The above equations are supplemented by a pair of constraint equations for for the scalars ${ }^{\|} E$ and $" B$ - see below. If one now assumes LRS, equations (24) and (25) reduce to

$$
" \dot{E}=2 \xi^{\|} B-\left(\frac{2}{3} \Theta-\Sigma\right) " E-{ }^{\|} j, \quad \| \dot{B}=-2 \xi^{\|} E-\left(\frac{2}{3} \Theta-\Sigma\right) " B,
$$

while the constraint equations are given by

$$
{ }^{\|} \hat{E}+{ }^{\|} E \Phi-2 \Omega^{\|} B-\varrho_{C}=0, \quad " \quad \hat{B}+{ }^{\|} B \Phi+2 \Omega^{\|} E=0 .
$$

As in this LRS one has that $E_{a}=E n_{a}, B_{a}=B n_{a}$ and $J_{a}=j n_{a}+\varrho_{C} u_{a}$, the superscript " can be dropped from the above equations without giving rise to any ambiguity.

Perfect fluid equations From the conservation of the energy-momentum tensor $\nabla^{a} T_{a b}=0$, it readily follows that

$$
u_{b} u_{a} \nabla^{a}(p+\rho)+(p+\rho)\left(u_{b}\left(\nabla^{a} u_{a}\right)+u_{a} \nabla^{a} u_{b}\right)+\nabla_{b} p+\left(\nabla^{a} F_{a c}\right) F_{b}^{c}=0 .
$$

In what follows, we will consider the projections of equation 28 along the directions parallel and orthogonal to the flow lines of the fluid. Contracting equation 28 with $u^{b}$ we obtain the conservation equation

$$
u_{a} \nabla^{a} \rho+(p+\rho) \nabla^{a} u_{a}-u^{b} F_{b}^{c}\left(\nabla^{a} F_{a c}\right)=0 .
$$

Now, in the case of an ideal conducting fluid, where $E_{a}=F_{a b} u^{b}=0$, the last term of equation 29 is identically zero and the electromagnetic field does not have a direct effect on the conservation equation along the flow lines. Contracting equation 28 with $h^{b c}$ one obtains the so-called Euler equation

$$
(p+\rho) u^{a} \nabla_{a} u^{c}+h^{b c} \nabla_{b} p+\left(\nabla^{a} F_{a d}\right) F_{b}{ }^{d} h^{b c}=0 .
$$

This last equation can also be written as

$$
(p+\rho) u_{a} \nabla^{a} u_{b}+\left(u_{b} u^{d} \nabla_{d}+\nabla_{b}\right) p+\left(\nabla^{a} F_{a d}\right)\left(F_{b}{ }^{d}+F^{e d} u_{e} u_{b}\right)=0 .
$$

The last term of equation (31) is identically zero as a consequence of the Maxwell equation so that at the end of the day one obtains the simpler expression $(p+\rho) u^{a} \nabla_{a} u^{c}+h^{b c} \nabla_{b} p+\left(\nabla^{a} F_{a d}\right) F^{c d}=0$. Now, using equation (21) in the above one obtains that

$$
\begin{aligned}
n^{c}\left(n^{b} \nabla_{b} p\right)+ & (\rho+p)\left(" \mathcal{A} n^{c}+{ }^{\perp} \mathcal{A}^{c}\right)+N_{c}^{b} \nabla_{b} p-"{ }^{\|} E\left(n^{c} \varrho_{C}+"{ }^{\|} j u^{c}\right) \\
& -{ }^{\perp} E^{c} \varrho_{C}-{ }^{\perp} j_{d}{ }^{\perp} E^{d} u^{c}-{ }^{\|} B \epsilon^{c d \perp} j_{d}-\left(\epsilon^{c d u \perp} j_{d}-" \| j \epsilon^{c u}\right)^{\perp} B_{u}=0, .
\end{aligned}
$$

Consequently, projecting equation (32) along $n_{a}$ we obtain a constraint equation for the pressure $p$. Namely, one has that

$$
\hat{p}+(p+\rho) \mathcal{A}-\varrho_{C}{ }^{\|} E-\epsilon^{d e \perp} j_{d}{ }^{\perp} B_{e}=0, \quad \dot{\rho}+(\rho+p) \Theta-{ }^{\|} E^{\|} j-{ }^{\perp} j^{c \perp} E_{c}=0 .
$$

where 29] has been considered for the conservation equation. In the particular case of a LSS, equation (33) read

$$
\dot{\rho}+(\rho+p) \Theta-{ }^{\|} E^{\|} j=0, \quad \hat{p}+(p+\rho) \mathcal{A}-\varrho_{C}{ }^{\|} E=0 .
$$


Evolution equation for the electric part of the Weyl tensor Using the expressions obtained in the previous Section it can be verified that the evolution equation for the electric part of the Weyl tensor takes the form

$$
\begin{aligned}
\| \dot{\mathcal{E}}=-\frac{1}{2}(\rho+ & p) \Sigma+\left(\frac{3}{2} \Sigma-\Theta\right){ }^{\|} \mathcal{E}+3^{\|} \mathcal{B} \xi+\epsilon^{c d} \delta_{c} \mathcal{B}_{d}+\epsilon^{c d \perp} \mathcal{B}_{d b} \zeta_{c}{ }^{b} \\
& +\Sigma^{a} \mathcal{E}_{a}-\Sigma_{c}^{e \perp} \mathcal{E}_{e}^{c}+2 \mathcal{A}_{c} \epsilon^{c d \perp} \mathcal{B}_{d}+\Omega_{c} \epsilon^{c d} \mathcal{E}_{d}+2 \mathcal{E}^{c} \alpha_{c}+\dot{\mathrm{F}}^{\mathrm{em}}
\end{aligned}
$$

where

$$
\dot{\mathrm{F}}^{\mathrm{em}}=n^{a} n^{b}\left(-\frac{1}{3} \sigma_{a b}\left(E^{2}+B^{2}\right)-\frac{1}{2} \dot{P}_{a b}-\frac{1}{6} \Theta P_{a b}-\frac{1}{2} D_{\langle a} \mathcal{G}_{b\rangle}-\mathcal{A}_{\langle a} \mathcal{G}_{b\rangle}-\frac{1}{2} \sigma_{\langle a}^{c} P_{b\rangle c}+\frac{1}{2} \epsilon_{c d\langle a} \omega^{c} P_{b\rangle}^{d}\right) .
$$

Now, following Clarkson's procedure for the $1+1+2$-decomposition of a LSS, equation (35) becomes

$$
{ }^{\|} \dot{\mathcal{E}}=\left(\frac{3}{2} \Sigma-\Theta\right){ }^{\|} \mathcal{E}+3 \xi^{\|} \mathcal{B}-\frac{1}{2}(\rho+p) \Sigma-\frac{1}{3}\left({ }^{\|} E^{2}+{ }^{\|} B^{2}\right)\left(\frac{\Sigma}{2}-\frac{\Theta}{3}\right)+\frac{1}{3} u^{a} \nabla_{a}\left({ }^{\|} E^{2}+{ }^{\|} B^{2}\right),
$$

while the constraint equation is given by

$$
\left\|\widehat{\mathcal{E}}+\frac{3}{2}\right\| \mathcal{E} \Phi-\frac{1}{3} \hat{\rho}-3 \Omega^{\|} \mathcal{B}-\mathrm{F}^{\mathrm{em}}=0, \quad \mathrm{~F}^{\mathrm{em}}=\frac{1}{2}\left[\left({ }^{\|} E^{2}+{ }^{\|} B^{2}\right) \phi+{ }^{\|} E^{\|} \widehat{E}+{ }^{\|} B \widehat{ } \widehat{B}\right] .
$$

Using, respectively, the evolution equations (26) and the equation for the electric parts of the Maxwell field magnetic field we obtain

$$
u^{a} D_{a} \mathcal{E}+\frac{2}{3} E j+\Theta\left[\frac{1}{3}\left(B^{2}+E^{2}\right)+\mathcal{E}\right]-3 \mathcal{B} \xi+\left[\frac{1}{2}(p+\rho)-\frac{1}{2}\left(B^{2}+E^{2}\right)-\frac{3}{2} \mathcal{E}\right] \Sigma=0 .
$$

Moreover, the constraint equation is given by

$$
n^{a} D_{a} \mathcal{E}-n^{a} D_{a} p-\frac{1}{3} n^{a} D_{a} \rho=\mathcal{A}(p+\rho)-\frac{1}{2}\left[\left(B^{2}+E^{2}+3 \mathcal{E}\right) \Phi-6 \mathcal{B} \Omega\right] .
$$

Notice that this last equation involves also the derivatives of $\rho$ and the pressure $p$ projected along the radial direction.

Evolution equation for the magnetic part of the Weyl tensor The evolution equation for the magnetic part of the Weyl tensor is given by

$$
\| \dot{\mathcal{B}}=\left(\frac{3}{2} \Sigma-\Theta\right) " \mathcal{B}-3^{\|} \mathcal{E} \xi+2 \bar{B}^{c} \alpha_{c}-\epsilon^{c d} \delta_{c} \mathcal{E}_{d}+\Sigma^{a} \mathcal{B}_{a}-\Sigma_{a c}{ }^{\perp} B^{a c}-2 \epsilon_{c d} \mathcal{A}^{c} \mathcal{E}^{d}+\epsilon^{c d} B_{d} \Omega_{c}-\frac{1}{2} \epsilon^{c d \perp} \mathcal{E}_{d}^{b} \zeta_{c b}+\dot{\mathrm{B}}^{\mathrm{em}},
$$

where

$$
\dot{\mathrm{B}}^{\mathrm{em}}=n^{a} n^{b}\left(\frac{1}{2} \operatorname{curl} P_{a b}+\frac{3}{2} \omega_{\langle a} \mathcal{G}_{b\rangle}+\frac{1}{2} \epsilon_{c d\langle a} \sigma_{b\rangle}^{c} \mathcal{G}^{d}\right) .
$$

Thus, in a LSS one obtains the propagation equation and the associated constraint equation

$$
\left\|\dot{\mathcal{B}}=\left(\frac{3}{2} \Sigma-\Theta\right){ }^{\|} \mathcal{B}-3 \xi^{\|} \mathcal{E}-\xi\left(" E^{2}+{ }^{\|} B^{2}\right), \quad "\right\| \widehat{\mathcal{B}}+\frac{3}{2} \| \mathcal{B} \Phi+(\rho+p) \Omega+3 \Omega^{\|} \mathcal{E}-\Omega\left({ }^{\|} E^{2}+{ }^{\|} B^{2}\right)=0 .
$$

Evolution equations for the kinematic quantities In order to discuss the evolution equations it is convenient to consider the following third rank tensors $R_{a b c}^{(n)} \equiv 2 \nabla_{[a} \nabla_{b]} n_{c}-R_{a b c d} n^{d}=0, \quad R_{a b c}^{(u)} \equiv 2 \nabla_{[a} \nabla_{b]} u_{c}-R_{a b c d} u^{d}=0$. The required evolution and associated constraint equations are then obtained from the projection of these zero quantities along the tensors $\left(u^{a}, n^{a}, \epsilon_{a b}, \epsilon_{a b c}\right)$, we are able to find out the evolution and constraint equations for the kinematic quantities.

From the constraint $R_{a b c}^{(u)} u^{a} \epsilon^{b c}=0$ we readily deduce the following evolution equation for the vorticity projected along the radial direction $\dot{\Omega}-\mathcal{A} \xi-\frac{1}{2} \epsilon^{b c} D_{b} \mathcal{A}_{c}=\left(\Sigma-\frac{2}{3} \Theta\right) \Omega+\left({ }^{\perp} \Sigma_{b}+\alpha_{b}\right)^{\perp} \Omega^{b}$. In the LSS case it readily reduces to

$$
\dot{\Omega}=\mathcal{A} \xi+\left(\Sigma-\frac{2}{3} \Theta\right) \Omega, \quad n^{a} D_{a} \Omega+(\Phi-\mathcal{A}) \Omega=0 .
$$

we obtain (in a LSS) the constraint equation through the contraction $R_{a b c}^{(u)} \epsilon^{a b c}=0$. Computing $u^{a} N^{b c} R_{a b c}^{(n)}=0$ we find an evolution equation for $\Phi$, namely,

$$
\begin{gathered}
\dot{\Phi}-\left(\frac{1}{3} \Theta-\frac{1}{2} \Sigma\right)(2 \mathcal{A}-\Phi)-2 \xi \Omega=\delta_{c} \alpha^{c}+\mathcal{A}^{d}\left(\epsilon_{d c}{ }^{\perp} \Omega^{c}-{ }^{\perp} \Sigma_{d}-a_{d}+\alpha_{d}\right) \\
-\zeta^{c d \perp} \Sigma_{c d}+a^{c}\left({ }^{\perp} \Sigma_{c}-\epsilon_{c u}{ }^{\perp} \Omega^{u}\right)+\epsilon_{u v}{ }^{\perp} E^{u \perp} B^{v}
\end{gathered}
$$


which, for a LSS, reduces to

$$
\dot{\Phi}-\left(\frac{1}{3} \Theta-\frac{1}{2} \Sigma\right)(2 \mathcal{A}-\Phi)-2 \xi \Omega=0, \quad n^{a} D_{a} \Phi+\mathcal{E}-\frac{2}{9} \Theta^{2}-2 \xi^{2}+\frac{2}{3} \rho-\frac{1}{3} \Theta \Sigma+\Sigma^{2}+\frac{1}{2} \Phi^{2}=0
$$

constraint equation for $\Phi$ in a LSS is found by the contraction $R_{a b c}^{(n)} n^{a} N^{b c}$. Computing the contraction $u^{a} \epsilon^{b c} R_{a b c}^{(n)}=0$ one obtains

$$
\dot{\xi}=\frac{1}{2} \mathcal{B}-\left(\frac{1}{3} \Theta-\frac{1}{2} \Sigma\right) \xi+\frac{1}{2} \epsilon_{a b} \delta^{a} \alpha^{b}+\left(\mathcal{A}-\frac{1}{2} \Phi \Omega\right) \Omega-\frac{1}{2} \epsilon_{a b} \zeta_{u}^{a \perp} \Sigma^{b u}+\frac{1}{2}\left[\epsilon_{a b}\left({ }^{\perp} \Sigma^{b}+\alpha^{b}\right)+{ }^{\perp} \Omega_{a}\left[\left(a^{a}+\mathcal{A}^{a}\right),\right.\right.
$$

which, for a LSS reduces to

$$
\dot{\xi}=\frac{1}{2} \mathcal{B}-\left(\frac{1}{3} \Theta-\frac{1}{2} \Sigma\right) \xi+\left(\mathcal{A}-\frac{1}{2} \Phi\right) \Omega, \quad n^{a} D_{a} \xi+\xi \Phi-\left(\frac{1}{3} \Theta+\Sigma\right) \Omega=0,
$$

the associated constraint is given, for a LSS, by the constraint $R_{a b c}^{(n)} \epsilon^{b c a}=0$. Through similar calculations, one obtains from the contractions $R_{a b c}^{(u)} u^{a} g^{a b}=0$ and $R_{a b c}^{(n)} u^{a} n^{b} u^{c}=0$ two alternative equations for the expansion scalar:

$$
\begin{aligned}
& u^{a} D_{a} \Theta-n^{a} D_{a} \mathcal{A}+\frac{1}{2}\left(B^{2}+E^{2}\right)+\frac{1}{2}(3 p+\rho)+\frac{1}{3} \Theta^{2}+\frac{3}{2} \Sigma^{2}-\mathcal{A}^{2}-\mathcal{A} \Phi-2 \Omega^{2}=0, \\
& \frac{1}{3} u^{a} D_{a} \Theta+u^{a} D_{a} \Sigma-n^{a} D_{a} \mathcal{A}=\mathcal{A}^{2}-\frac{1}{2}\left(B^{2}+E^{2}\right)-\mathcal{E}-\frac{1}{2}\left(p+\frac{1}{3} \rho\right)-\left(\frac{1}{3} \Theta+\Sigma\right)^{2} .
\end{aligned}
$$

Finally, in the sequel it will be useful to consider the following equations obtained, respectively, from the contractions $R_{a b c}^{(n)} \epsilon^{a b} u^{c}=0, R_{a b c}^{(u)} u^{a} N^{b c}=0, R_{a b c}^{(u)} n^{a} N^{b c}=0$ :

$$
\begin{aligned}
& \mathcal{B}-3 \xi \Sigma+(2 \mathcal{A}-\Phi) \Omega=0, \\
& \frac{2}{3} u^{a} D_{a} \Theta-u^{a} D_{a} \Sigma+p+\frac{1}{3} \rho+2\left(\frac{1}{3} \Theta-\frac{1}{2} \Sigma\right)^{2}-\mathcal{E}-\mathcal{A} \Phi-2 \Omega^{2}=0, \\
& \frac{2}{3} n^{a} D_{a} \Theta-n^{a} D_{a} \Sigma-\frac{3}{2} \Sigma \Phi-2 \xi \Omega=0 .
\end{aligned}
$$

\section{REMARKS ON THE THERMODYNAMICAL QUANTITIES}

In the present article we consider a one species particle fluid (simple fluid), and denote, respectively, by $n, s, T$ the particle number density, the entropy per particle and the absolute temperature, as measured by comoving observers. We also introduce the volume per particle $v$ and the energy per particle $e$ via the relations $v \equiv 1 / n$ and $e \equiv \rho / n$. In terms of these variables the first law of Thermodynamics, $\mathrm{d} e=-p \mathrm{~d} v+T \mathrm{~d} s$, takes the form

$$
\mathrm{d} \rho=\frac{p+\rho}{n} \mathrm{~d} n+n T \mathrm{~d} s .
$$

Assuming an equation of state of the form $\rho=f(n, s) \geq 0$, one obtains from equation 477 that

$$
p(n, s)=n\left(\frac{\partial \rho}{\partial n}\right)_{s}-\rho(n, s), \quad T(n, s)=\frac{1}{\rho}\left(\frac{\partial \rho}{\partial s}\right)_{n} .
$$

Assuming that $\partial p / \partial \rho>0$ we define the speed of sound $\nu_{s}=\nu_{s}(n, s)$ by

$$
\nu_{s}^{2} \equiv\left(\frac{\partial p}{\partial \rho}\right)_{s}=\frac{n}{\rho+p} \frac{\partial p}{\partial n}>0 .
$$

Since we are not considering particle annihilation or creation processes we consider the equation of conservation of particle number:

$$
u^{a} \nabla_{a} n+n \nabla_{a} u^{a}=0 .
$$

Combining this equation with equations 29 and 47 we obtain

$$
u^{a} \nabla_{a} s=\frac{1}{n T} u^{b} F_{b}^{c} \nabla^{a} F_{a c}
$$


Where $n$ is subject to equation (50) and $T$ is given by equation (48), thus, using the $1+1+2$ decompositionin a LSS one obtain the couple of equations

$$
\dot{s}=+\frac{1}{n T} \| E^{\|} j \quad \dot{n}+n^{\|} \Theta=0 .
$$

In the case of an infinitely conducting plasma, where the last term of equation (47) vanishes, equation (51) describes an adiabatic flow - that is, $u^{a} \nabla_{a} s=0$, so that the entropy per particle is conserved along the flow lines. A particular case of interest is when $s$ is a constant of both space and time. In this case the equation of state can be given in the form $p=p(\rho)$. In the following for convenience, it is assumed that $\nabla_{a} p=v_{s} \nabla_{a} \rho$ where we have defined $v_{s} \equiv \nu_{s}^{2}$. Further discussion on the thermodynamical quantities and assumptions can be found in Appendix C.

\section{VARIABLES AND EQUATIONS}

In this subsection we summarise our analysis so far. The evolution equations for the independent components of the vector-valued unknown

$$
\vec{v}=(\rho, s, n, \Omega, \xi, \Phi, E, B, \mathcal{B}, \mathcal{E}, \Theta, \Sigma, \mathcal{A}),
$$

describing the evolution of a LSS in equations (34), (52), 42, , 44), 433, 26, ,41, , 38, ,45a) and 45b are given by

$$
\begin{aligned}
& \dot{\rho}=-(\rho+p) \Theta+E^{\|} j, \\
& \dot{s}=\frac{1}{n T} E^{\|} j, \\
& \dot{n}=-n \Theta \\
& \dot{\Omega}=\mathcal{A} \xi-\frac{2}{3} \Theta \Omega+\Sigma \Omega, \\
& \dot{\xi}=\frac{1}{2} \mathcal{B}-\left(\frac{1}{3} \Theta-\frac{1}{2} \Sigma\right) \xi+\left(\mathcal{A}-\frac{1}{2} \Phi\right) \Omega, \\
& \dot{\Phi}=\left(\frac{1}{3} \Theta+\frac{1}{2} \Sigma\right)(2 \mathcal{A}+\Phi)-2 \xi \Omega=0, \\
& \dot{E}=2 \xi B-\left(\frac{2}{3} \Theta-\Sigma\right) E-{ }^{\|} j, \\
& \dot{B}=-2 \xi E-\left(\frac{2}{3} \Theta-\Sigma\right) B \\
& \dot{\mathcal{B}}=\left(\frac{3}{2} \Sigma-\Theta\right) \mathcal{B}-3 \xi \mathcal{E}-\xi\left(E^{2}+B^{2}\right), \\
& \dot{\mathcal{E}}=\left(\frac{3}{2} \Sigma-\Theta\right) \mathcal{E}+3 \xi \mathcal{B}-\frac{1}{2}(\rho+p) \Sigma+\left(\frac{\Sigma}{2}-\frac{\Theta}{3}\right)\left(E^{2}+B^{2}\right)-\frac{2}{3} E^{\|} j, \\
& u^{a} D_{a} \Theta-n^{a} D_{a} \mathcal{A}=-\frac{1}{2}\left(B^{2}+E^{2}\right)-\frac{1}{2}(3 p+\rho)-\frac{1}{3} \Theta^{2}-\frac{3}{2} \Sigma^{2}+\mathcal{A}^{2}+\mathcal{A} \Phi+2 \Omega^{2}, \\
& u^{a} D_{a} \Sigma+\frac{1}{3} u^{a} D_{a} \Theta-n^{a} D_{a} \mathcal{A}=\mathcal{A}^{2}-\frac{1}{2}\left(B^{2}+E^{2}\right)-\mathcal{E}-\frac{1}{2}\left(p+\frac{1}{3} \rho\right)-\left(\frac{\Theta}{3}+\Sigma\right)^{2} .
\end{aligned}
$$

In the subsequent stability analysis, we will assume a vanishing radial acceleration. It is worth pointing out that the derivative along the radial direction of the acceleration $\mathcal{A}$ appears in the evolution equations for $\Theta$ and $\Sigma$. In Section $\mathrm{VA}$ and in Appendices $\mathrm{A} \mathrm{B}$ and $\mathrm{C}$ we provide a suitable propagation equation for this variable so as to complete a symmetric hyperbolic evolution system.

As discussed in more detail in Section IV] the system will be supplemented by an equation of state of the form $p=p(\rho)$. Furthermore, it will be necessary to specify the form of the conduction current, $j^{a}$. Consistent with Ohm's law, we assume a linear relation between the conduction current $j^{a}$ and the electric field. More precisely, we set $j^{a}=\sigma^{a b} E_{b}$, where $\sigma^{a b}$ denotes the conductivity of the fluid (plasma). We will restrict our attention to isotropic fluids for which $\sigma^{a b}=\sigma_{J} g^{a b}$, so that

$$
J^{a}=\varrho_{C} u^{a}+\sigma_{J} E^{a},
$$

with $\sigma_{J}$ the electrical conductivity coefficient. In terms of a $1+1+2$-decomposition one has that in the particular case of a LSS

$$
" j^{a}={ }^{\|} E \sigma_{J} n^{a} .
$$

The system (54a)-(54j) is complemented by the constraint equations for the components of the vector-valued function $\vec{v}$. Some of these constraint equations were obtained in the previous Sections. Further discussion can be found in the 
Appendices to this article. As the primary aim of this work is the study of the time evolution and the analysis of its stability, the discussion of the constraint equations will remain in a secondary level. In what follows, it is assumed that the constraint equations are satisfied at all time. This assumption can be removed by fairly general arguments - see [23, 24].

\section{A. Re-parametrised set of evolution equations}

In what follows we assume that $\partial_{a} v_{s}=0$ and we introduce the variables $Q$ and $T$ via the relations

$$
Q \equiv T+3 \Sigma, \quad T \equiv \frac{2}{3} \Theta-\Sigma \quad \text { so that } \quad \Sigma=\frac{1}{3}(Q-T), \quad \Theta=\frac{3}{2}(T+\Sigma) .
$$

In terms of these one obtains a vector-valued unknown with components given by

$$
\mathbf{v} \equiv(\rho, E, B, \mathcal{E}, \mathcal{B}, Q, T, \xi, \Phi, \Omega, \mathcal{A})
$$

and equations 54a, 54g), 54h, 54j), 54i), 54f), 54e, and (54d) take the form

$$
\begin{aligned}
& \dot{\rho}-E j+\frac{3}{2}(p+\rho)\left(\frac{1}{3}(Q-T)+T\right)=0, \\
& \dot{E}-2 B \xi+E T+j=0 \\
& \dot{B}+B T+2 E \xi=0 \\
& \dot{\mathcal{E}}-3 \mathcal{B} \xi+\frac{1}{2}\left(B^{2}+E^{2}\right) T+\frac{3}{2} \mathcal{E} T+\frac{2}{3} E j+\frac{1}{6}(p+\rho)(Q-T)=0, \\
& \dot{\mathcal{B}}+\frac{3}{2} \mathcal{B} T+3 \mathcal{E} \xi+\left(B^{2}+E^{2}\right) \xi=0, \\
& \dot{\Phi}-\mathcal{A} T-2 \xi \Omega+\frac{1}{2} T \Phi=0, \\
& \dot{\xi}-\mathcal{A} \Omega+\frac{1}{2}(\xi T+\Phi \Omega-\mathcal{B})=0, \\
& \dot{\Omega}-\mathcal{A} \xi+T \Omega=0 .
\end{aligned}
$$

Moreover, from equation $46 \mathrm{a}$ one finds that $\mathcal{B}-3 \mathcal{A} \xi+(2 \mathcal{A}-\Phi) \Omega=0$, while from equations 541 and $54 \mathrm{k}$ one obtains that

$$
\begin{aligned}
& \dot{T}-\mathcal{A} \Phi-\mathcal{E}+p+\frac{1}{3} \rho+\frac{1}{2} T^{2}-2 \Omega^{2}=0 \\
& \dot{Q}-2 \hat{\mathcal{A}}-2 \mathcal{A}^{2}+B^{2}+E^{2}+2 \mathcal{E}+p+\frac{1}{3} \rho+\frac{1}{2} Q^{2}=0 \\
& \frac{4 \dot{\mathcal{A}}}{v_{s}}-2 \hat{Q}+\frac{4 E \hat{j}}{p+\rho}-\frac{4 E \dot{\varrho}_{C}}{v_{s}(p+\rho)}-\frac{2}{v_{s}(p+\rho)}\left(\mathcal{A}\left[Q\left(v_{s}-1\right)+2 T v_{s}\right](p+\rho)-2 \mathcal{A} E j\left(1+2 v_{s}\right)\right. \\
& +\varrho_{C}\left[E Q\left(2+v_{s}\right)+2\left(E T v_{s}+2 B \xi\right)\right]+v_{s}(p+\rho)[\Phi(Q-T)+4 \xi \Omega] \\
& \left.-2 j\left[\varrho_{C}\left(1+v_{s}\right)+v_{s}(2 B \Omega-E \Phi)\right]\right)=0 .
\end{aligned}
$$

As already discussed in Sections $\left[\mathrm{IV}\right.$ and $\sqrt{\mathrm{V} p}$ the fields $p$ and $\varrho_{C}$ can be regarded as functions of $\rho$, and while $j_{c}$ is regarded as a function of the electric field $E$. Equations (59a)- $60 \mathrm{~b}$ do not contain explicit dependence on the derivative of the matter density along the radial direction. In equation (60c) the equation of state and the constraint equation have been used to obtain a simpler expression. The equation for the radial acceleration can be recovered after some further calculations - see the Appendix Section. In order to simplify the stability analysis of the subsequent Sections and to be able to extract some information from the rather complicated perturbed equations we will focus on the null radial acceleration case. Summarizing, our analysis has lead to a symmetric hyperbolic system consisting of eleven evolution equations for eleven scalar variables of the form

$$
\mathbf{A}^{t} \partial_{t} \mathbf{v}-\mathbf{A}^{j} \partial_{j} \mathbf{v}=\mathbf{B v}
$$

\section{PERTURBATIONS}

We will perform a perturbation to first order of the variables $\mathbf{v}$ so that $\mathbf{v} \mapsto \stackrel{\circ}{\mathbf{v}}+\epsilon \breve{\mathbf{v}}$ with the parameter $\epsilon$ setting the perturbation order that controls the size of perturbation and $\breve{\mathbf{v}}$ describing the perturbation of the background solution 
$\stackrel{\circ}{\mathbf{v}}$. Thus, assuming that the background variables $\stackrel{\circ}{\mathbf{v}}$ satisfy the evolution equations, and $\operatorname{defining}\left(\mathrm{d} \mathbf{v}^{(a)} / \mathrm{d} \epsilon\right)_{\epsilon=0} \equiv \breve{\mathbf{v}}^{(a)}$, we can obtain from equations $(59 \mathrm{a})-(59 \mathrm{~h})$ the following equations for the linear perturbations:

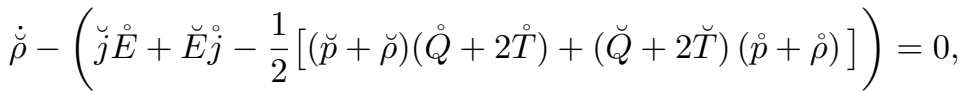

$$
\begin{aligned}
& \dot{\vec{E}}+\breve{j}-2 \breve{\xi} \stackrel{\circ}{B}+\breve{T} \stackrel{\circ}{E}+\breve{E} \stackrel{\circ}{T}-2 \breve{B} \dot{\xi}=0 \\
& \dot{\vec{B}}+\breve{T} \stackrel{\circ}{B}+2 \breve{\xi} \stackrel{\circ}{E}+\breve{B} \stackrel{\circ}{T}+2 \breve{E} \stackrel{\circ}{\xi}=0 \\
& \dot{\dot{\mathcal{E}}}-3 \breve{\xi} \mathcal{B}+\frac{1}{6} \breve{T}\left[3\left(\stackrel{\circ}{B}^{2}+\stackrel{\circ}{E}^{2}+3 \mathcal{\mathcal { E }}\right)-(\stackrel{\circ}{p}+\stackrel{\circ}{\rho})\right]+\frac{2}{3} \breve{j} \stackrel{\circ}{E}+\frac{1}{3} \breve{E}(2 \stackrel{\circ}{j}+3 \stackrel{\circ}{E} \stackrel{\circ}{T})+\frac{1}{6} \breve{Q}(\stackrel{\circ}{p}+\stackrel{\circ}{\rho})+\frac{1}{6}(\breve{p}+\breve{\rho})(\stackrel{\circ}{Q}-\stackrel{\circ}{T}) \\
& +\frac{3}{2} \breve{\mathcal{E}} \stackrel{\circ}{T}-3 \breve{\mathcal{B}} \dot{\xi}+\breve{B} \stackrel{\circ}{\circ} \stackrel{\circ}{T}=0, \\
& \dot{\dot{\mathcal{B}}}+\frac{3}{2} \breve{T} \dot{\mathcal{B}}+\breve{\xi}\left(\stackrel{\circ}{B}^{2}+3 \mathcal{\mathcal { E }}\right)+\frac{3}{2} \breve{\mathcal{B}} \stackrel{\circ}{T}+3 \breve{\mathcal{E}} \stackrel{\circ}{\xi}+2 \breve{B} \stackrel{\circ}{B} \dot{\xi}=0
\end{aligned}
$$

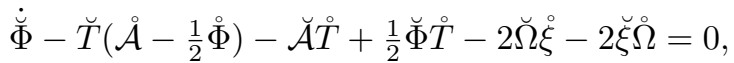

$$
\begin{aligned}
& \dot{\xi}-\frac{1}{2} \breve{\mathcal{B}}-\breve{\Omega}\left(\stackrel{\mathcal{A}}{-}-\frac{1}{2} \stackrel{\circ}{\Phi}\right)+\frac{1}{2} \breve{\xi} \stackrel{\circ}{T}+\frac{1}{2} \breve{T} \dot{\xi}-\breve{\mathcal{A}} \stackrel{\Omega}{\Omega}+\frac{1}{2} \breve{\Phi} \stackrel{\Omega}{\Omega}=0, \\
& \dot{\check{\Omega}}-\breve{\xi} \stackrel{\circ}{\mathcal{A}}+\breve{\Omega} \stackrel{\circ}{\mathrm{T}}-\breve{\mathcal{A}} \dot{\xi}+\breve{T} \stackrel{\Omega}{\Omega}=0 .
\end{aligned}
$$

In addition one obtains from (46a) the linearised constraint $\breve{\mathcal{B}}+\breve{\mathcal{A}}(2 \stackrel{\Omega}{\Omega}-3 \dot{\xi})-3 \stackrel{\mathcal{A}}{\xi}-\breve{\Phi} \stackrel{\Omega}{\Omega}+\breve{\Omega}(2 \stackrel{\mathcal{A}}{-}-\stackrel{\circ}{\Phi})=0$, while perturbing to first order equations $(60 \mathrm{a})-(60 \mathrm{~b})$ ) describing the evolution of the variables $T$ and $Q$ we obtain

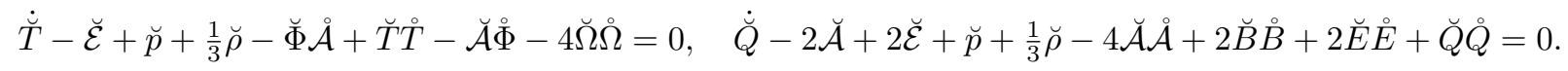

Similar calculations render a complicated linearised equation for the perturbation of acceleration $\mathcal{A}$. Thus, in order to undertake a stability analysis we make some assumptions about the configuration so as to obtain a simplified system. First, we will analyse the set $(59 \mathrm{a})-(60 \mathrm{~b})$ ) with the assumption $\mathcal{A}=0$. Thus, we do not consider any more equation 60c. Accordingly, we consider the simplified vector-valued unknown v $\equiv(\rho, E, B, \mathcal{E}, \mathcal{B}, Q, T, \xi, \Phi, \Omega)$.

\section{A. Remarks on the set of equations}

We consider the set of linearised evolution equations in the form

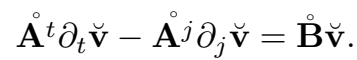

General theory of linear of partial differential equations shows that systems of this form can either converge (in an oscillating manner or exponentially) to constant values, have an asymptotic exponential (oscillating) decay or be unstable - see e.g. 45. In the rest of this article, we look at solutions in which the perturbations are stabilised on constant values or decay. In order to study the asymptotical behaviour of the characteristic solutions of perturbed system it is necessary the study of the eigenvalues of the matrix $\stackrel{\circ}{\mathbf{B}}$ as $t \rightarrow \infty$. Notice that in the the matrix-valued function $\stackrel{\circ}{\mathbf{B}}$ are, in general, functions of the coordinates. Under these circumstances, the core of the stability analysis consists on analysing whether the matrix $\stackrel{\circ}{\mathbf{B}}$ satisfies some appropriate relaxed stability eigenvalue conditions. More precisely, we study then the eigenvalue problem of $\stackrel{\circ}{\mathbf{B}}$ by looking at the sign of the real parts of the corresponding eigenvalues $\lambda_{i}$ (or modes of the perturbed system). A similar approach has been used in [24] see also discussion in [4]. General theory concerning the case where the coefficients linearised system are constant is discussed in [45]. The case of systems with vanishing eigenvalues has been discussed in 46 48] while for a general discussion on the time dependent case and the case of non constant matrix coefficient see [24].

Due to the form of the matrix $\stackrel{\circ}{\mathbf{B}}$ associated to the present problem, a fully analysis of the stability properties of this system is extremely cumbersome. We proceed by analysing the sign of the eigenvalues using the fact that a sufficient condition for the instability of the system is the existence of al last one positive-real part eigenvalues. For a more extended discussion concerning the requirements of the so-called of the relaxed stability eigenvalue condition see [46, 48. This simple requirement will provide a immediate way to show the conditions where instability occurs - namely, if there is at least one $i$ such that $\operatorname{Re}\left(\lambda_{i}\right)>0$. Crucially, in the discussion of the stability one does not need

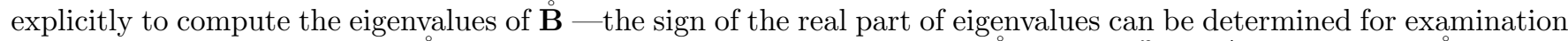
of the structure of the matrix $\mathbf{B}$. Given the characteristic polynomial $\mathfrak{P}\left(\stackrel{\mathbf{B}}{)}(x)=\sum_{i=0}^{n} c_{i} x^{i}\right.$ of the matrix $\mathbf{B}^{\circ}$, we will 
make use of the following well-known results on the roots sign: the trace of a matrix is equal to the sum of its eigenvalues, hence if the trace of the matrix is positive then the system is unstable. Now, recall that the determinant of a matrix is the product of the eigenvalues. Accordingly, a necessary condition for stability is that det $\mathbf{B}^{\circ} \neq 0$ and $(-1)^{n} \operatorname{det} \stackrel{\circ}{\mathbf{B}}>0$ where $n$ is the number of distinct eigenvalues. Other stability criteria based on inspecting the characteristic polynomial are the Routh-Hurwitz criterion and the Liénard-Chipart theorem - see 42 44. Wherever possible, we will also make use of the Descartes criterion to determine the maximum number of positive and negative real roots of the polynomial $\mathfrak{P}(\stackrel{\circ}{\mathbf{B}})(x)$ for $c_{i} \in \mathbb{R}$. In particularly simple cases one can exploit a generalisation of the Descartes rule considering the Routh-Hurwitz criterion to determine the number of roots with positive and negative real part of $\mathfrak{P}(\stackrel{\circ}{\mathbf{B}})(x)$ by constructing the associated Routh matrix.

In the case under consideration the matrix $\stackrel{\circ}{\mathbf{B}}$ has eleven columns and the coefficients of the characteristic polynomial are, unfortunately, large expressions without an obvious structure. Therefore, we approach the analysis of the stability problem making the following assumption: $\stackrel{\circ}{j}=\stackrel{\circ}{E} \sigma_{J}, \quad \breve{j}=\breve{E} \sigma_{J}, \quad \stackrel{\circ}{p}=\stackrel{\circ}{\rho} v_{s}, \quad \breve{p}=\breve{\rho} v_{s}$. Thus, we take the square of the sound velocity $v_{s}=\nu_{s}^{2}$ to be constant and assume Ohm's law $j=E \sigma_{J}$ with $\stackrel{\circ}{\sigma}_{j} \equiv \breve{\sigma}_{j}$ and restrict our attention to the case of null radial acceleration -i. e. $\mathcal{A}=0$.

Before proceeding to the analysis of particular cases, it is convenient to note here some general properties of the system of symmetric hyperbolic equations under consideration. The matrix $\mathbf{B}_{\mathbf{B}}$ can be explicitly written as the $10 \times 10$-matrix

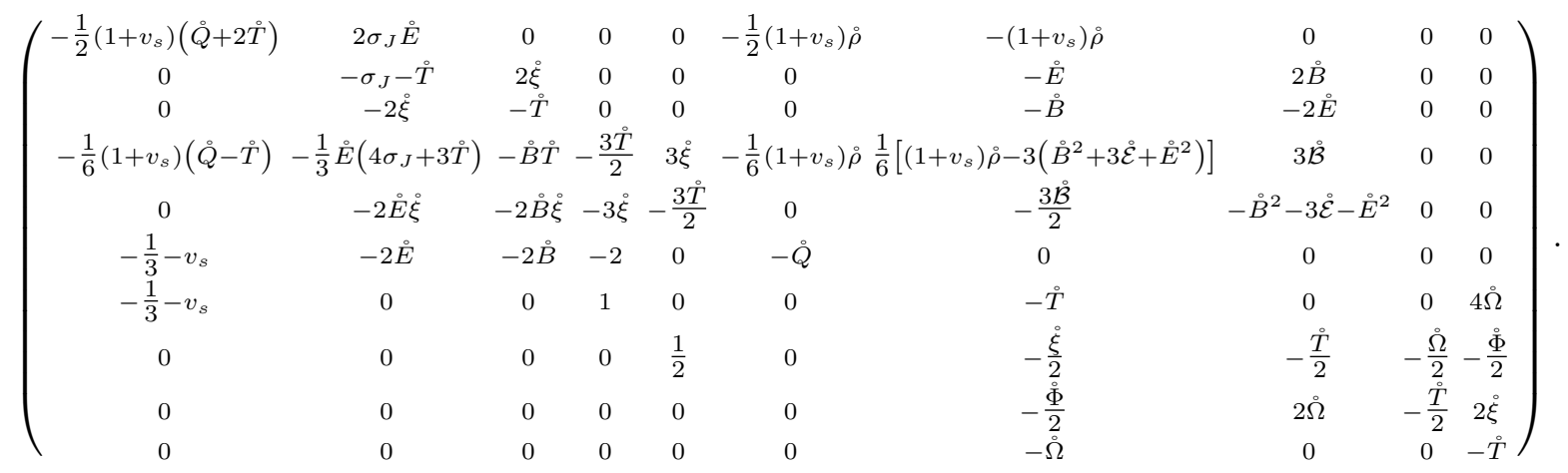

\section{DISCUSSION ON THE NONLINEAR STABILITY OF THE SYMMETRIC HYPERBOLIC SYSTEM}

In this Section we discuss necessary conditions for the nonlinear stability of the configuration under consideration. We focus on the reference solution described by matrix $\mathbf{B}_{\mathbf{B}}$ in 64 . We present the analysis of the system stability for some special configurations defined by proper assumptions on the kinematic variables given by the $1+1+2$ decomposition. Each case is then identified according to the assumptions that characterise it - the fields assumed to vanish are indicated in parenthesis. We discuss in details the instability condition for the complete list of cases. Assuming the radial acceleration and other kinematic variables to be zero, we explore the implications of the assumption on the set up configuration and its stability. A first insight into the stability of the system can be inferred from the trace in terms of $\Sigma$ and $\Theta$ as

$$
\operatorname{Tr} \stackrel{\circ}{\mathbf{B}}=6 \stackrel{\circ}{\Sigma}-\left(7+v_{s}\right) \stackrel{\circ}{\Theta}-\sigma_{J}
$$

It then readily follows that $\operatorname{Tr} \stackrel{\circ}{\mathbf{B}}>0$ so that the system is not stable if

$$
\Sigma>\frac{\left(7+v_{s}\right) \Theta+\sigma_{J}}{6} .
$$

In the remainder of this article, we proceed to a more detailed analysis in which we classify the results according to suitable assumptions on the remaining system kinematic variables $(T, Q, \Phi, \xi, \Omega)$. It should be pointed out, for ease of reference, that the condition $T=0$ is equivalent to the relation $\frac{2}{3} \Theta=\Sigma$ between the expansion $\Theta$ of the 3 -sheets and the radial part of the shear of the 3 -sheet $\Sigma$. Similarly, the condition $Q=0$ is equivalent to $\frac{1}{3} \Theta=-\Sigma$, while the two conditions $T=0$ and $Q=0$ imply $\Sigma=0$ and $\Theta=0$. We also notice from the Maxwell equations that the evolution of the electric (respectively, magnetic) field is coupled to the magnetic (respectively, electric) field via the twisting of the 2 -sheet. Hence, when $\xi=0$ the two evolution equations decouple and evolve with the only common dependence on $T$. 


\section{A. Preliminaries on the symmetries of the system and classes of solutions}

The configuration can expand or contract along the direction of symmetry, according to $\Theta>0$ or $\Theta<0$ respectively, but not accelerating as it is assumed the parallel acceleration $\mathcal{A}=0$. The limit situation of null radial acceleration has been adopted to simplify the analysis of the unstable modes, in this scheme we are able to provide a complete classification of the stable and unstable solutions for the Einstein-Maxwell-Euler system, considering appropriate restrictions for the set of the perturbed equations. All the kinematic quantities and fields are reduced to scalars by the projections in the radial direction and on the orthogonal plane by the projector $N_{a b}$, the set of scalars define the vector variable $\vec{v}$ introduced in Eq. (58). Any further restrictions on the system, as the vanishing of other dynamical variables:

$$
Q_{i} \in \vec{\nu}, \quad \vec{\nu} \equiv\{T, Q, \Phi, \xi, \Omega\} \subset \overrightarrow{\mathbf{v}}
$$

leads to particular different solutions of the Einstein-Maxwell-Euler equations with the new symmetry conditions. The self-gravitating systems will be especially constrained by the couple $(\mathcal{E}, \mathcal{B})$, in the gravitating systems the background geometry is assumed to be in one of the classes of solutions including the new symmetries. The vector $\vec{\nu}$ is actually a restriction of the vector variable $\overrightarrow{\mathbf{v}}$, where the fluid four-velocity $u_{a}$ defines the metric in its $3+1$ form and supplies the projected components of the Weyl tensor together with the direction of symmetry $(\xi, \Phi)$ related through Eq. $(9)$, and the variation of the symmetry direction $n_{a}$, used to construct the metric tensor in its $2+1+1$ form. The vanishing of the $Q_{i}$ elements implies further restrictions involving the annihilation of other quantities, or the constance of $Q_{j} \neq Q_{i}$ during the evolution along $u_{a}$. We can then refer to the general conditions $\mathfrak{C}$ :

$$
\begin{gathered}
\mathfrak{C} \quad\left(Q_{i}=0\right): \quad\left(E^{2}, B^{2}, \mathcal{E}, \Omega\right) \mapsto\left(p+\frac{\rho}{3}\right) \\
\left(Q_{j}, \mathcal{B}\right)=0 \\
\dot{Q}_{j}=0 .
\end{gathered}
$$

Conditions in Eqs. (69), specified in Table (I), define five principal classes of solutions according to the assumption of null $Q_{i} \in \vec{\nu}$. Table (II) shows the sub-classes of solutions constructed by the vanishing of a couple of scalars $\left(Q_{i}, Q_{j}\right)$. Conditions (68) are listed in Table (III), and state the relationship between the remaining field variables of the vector $\overrightarrow{\mathbf{v}}$ i.e. the couple $(\mathcal{E}, \mathcal{B})$, the electromagnetic fields $(E, B)$, and the matter density $\rho$. Finally, condition $(70)$ is made explicit in Table (IV). Tables (I), (II), (III) and (IV) characterize entirely the system throughout its sub-configurations ruled by the system symmetries, providing a complete classification of the solutions. An analysis and a general discussion of the solutions of the Einstein-Maxwell system in terms of the magnetic and electric parts of the Weyl tensor can be found in 39. The stability analysis will be performed on the systems with $\mathcal{A}=0$, on the five classes of configurations and their sub-classes. The $\mathfrak{I}$-class with $(\mathcal{A T})$ and $\mathfrak{I} \mathfrak{I}$-class with $(\mathcal{A Q})$ are particularly significant. Systems $T=0$ are characterized by $\Theta \Sigma>0$, i.e. the sign of the expansion is concordant with that of the radial shear, then positive for expanding systems, along the direction of symmetry, or negative for contracting systems, see also Fig. (1). On the other side systems $Q=0$ correspond to the case $\Theta \Sigma<0$. The relative sign of the scalars of the radial shear and expansion is a significant element affecting the stability of the system and the equilibrium configurations: even in the general case where the only assumption on the system is the null radial acceleration $\mathcal{A}=0$, the balance of the contributions given by the radial shear in the systems in expansion $(\Theta>0)$ or in contraction $(\Theta<0)$, is relevant in determining the states certainly unstable. For the contracting systems, or in expansion but with positive shear, a threshold on the expansion rate $\Theta$ or equivalently the radial shear, appears for the emergence of the instability, this is a function of the two model parameters $\left(\sigma_{J}, v_{s}\right)$. The magnetic field, although it is not constant in the time along the direction of symmetry, has no specific role to establish the stability of this model. It is possible to show that for the case $(\mathcal{A T})$ there is always a threshold for the emergence of unstable phases, while in the case of the expanding systems with negative shear, the difference in sign between the two scalar does not involve any instability threshold.

The conditions for the equilibrium of these solutions involve quantities $Q_{i} \in \vec{\nu}$ exclusively related to the fluid dynamics such as $\mathcal{A} T \xi$ or $\mathcal{A} \xi \Omega$ and imply a serious constraint on the background, for that instability is in some cases certainly verified assuming that $\mathcal{B}=0$. Table (III) shows conditions $\mathfrak{C}$ in Eqs. 68): only the electric part of the Weyl tensor is determined by the matter fields and the vorticity. In general the classes $(\mathcal{A} \mathbf{T})$ and $(\mathcal{A Q})$ do not implies the vanishing of others variables by conditions $\mathfrak{C}$ in Eqs. $(69)$ : that is, the systems do not require any additional symmetry as the initial data $Q_{j}=0$ for the solutions 68 . Remarkably the $(\mathcal{A Q T})$ class corresponds to the case of null shear and null radial expansion. Tables (II) and (II) show that the couples $(\xi, \Omega)$ and $(\xi, \Phi)$ are related. The magnetic field must be constant along the fluid flow for the solution $(\mathcal{A}, T, \xi)$. and conversely $\mathcal{B}=0$ where $\xi$ is zero. The solutions $T=0$ have radial vorticity $\Omega$ constant during the evolution of the system as shown in Table (IV). If the radial vorticity is initially zero, then the expansion $\Phi$ of the 2 -sheet generated by $n_{a}$ is constant along $u_{a}$ and the electric part of the Weyl tensor is entirely determined by the matter field $\rho$. If initially $\Phi=0$ then $\xi=0$ and therefore $\mathcal{B}=0$, providing finally a no vacuum solution with null magnetic component of the Weyl tensor, Table (III). 


\begin{tabular}{|c|c|c|c|c|}
\hline & $\mathcal{A}$ & $\mathcal{A} T$ & $\mathcal{A Q}$ & $\mathcal{A} \xi$ \\
\hline II-class & $\mathcal{A} T$ & $\bar{\nabla}$ & $\bar{\checkmark}$ & $\bar{\nabla}$ \\
\hline II-class & $\mathcal{A} Q$ & $\mathcal{A T Q}$ & $\checkmark$ & $\checkmark$ \\
\hline IIII-class & $\begin{aligned} \mathcal{A} \Phi= & \mathcal{A} \Phi \Omega \\
& \mathcal{A} \Phi \xi \mathcal{B}\end{aligned}$ & $\left|\begin{array}{r}\mathcal{A} T \Phi=T+\mathcal{A} \Phi \Omega \\
T+\mathcal{A} \Phi \xi \mathcal{B}\end{array}\right|$ & $\left|\begin{array}{r}\mathcal{A} Q \Phi=Q+\mathcal{A} \Phi \Omega \\
Q+\mathcal{A} \Phi \xi \mathcal{B}\end{array}\right|$ & $\checkmark$ \\
\hline $\mathfrak{I V}$-class & $\mathcal{A} \xi$ & $|\mathcal{A} T \xi=T+\mathcal{A} \Phi \xi \mathcal{B}|$ & $\mathcal{A} Q \xi$ & $\checkmark$ \\
\hline $\mathfrak{V}$-class & $\mathcal{A} \Omega$ & $\mathcal{A} T \Omega$ & $\mathcal{A} Q \Omega$ & $\mathcal{A} \xi \Omega=\mathcal{A} \xi \Omega \mathcal{B}$ \\
\hline
\end{tabular}

TABLE I: Null radial acceleration $\mathcal{A}$ : the five principal classes of solutions for the linear stability the problem, identified by canceling the radial acceleration and a scalar quantity of the set $Q_{i} \in \vec{\nu}=\{T, Q, \Phi, \xi, \Omega\}$ and $\mathcal{B}$, the magnetic part of the Weyl tensor. The elements of the Table explicit conditions $\mathfrak{C}$ in 69 . Thus, for example the $\mathfrak{I} \mathfrak{I} \mathfrak{I}$-class, conditions of $(\mathcal{A} \boldsymbol{\Phi})$ configurations implies a null radial vorticity $\Omega$ or the null couple $\xi$ and $\mathcal{B}$. Then we assumed that the system has a further constraint represented by a third zero quantity $Q_{i} \in \vec{\nu}$ defining the subclasses. The check marks indicate the cases already treated, the Table exhausts the five classes $\left(\mathcal{A}, \mathbf{Q}_{\mathbf{i}}\right)$ and the subclasses $\left(\mathcal{A}, Q_{i}, Q_{j}\right)$. Particularly the $\mathfrak{I} \mathfrak{I} \mathfrak{I}-$ class $(\mathcal{A} \phi)$ shows the symmetry between the solutions at zeros $(\mathcal{A Q}),(\mathcal{A T})$ and $\{\xi \Omega \mathcal{B}\}$. Subclasses, $\left(\mathcal{A}, Q_{i}, Q_{j}, Q_{k}\right)$ are in Table. [II.

\begin{tabular}{|c|c|c|c|}
\hline $\mathcal{A} T Q$ & $\mathcal{A} T \xi$ & $\mathcal{A} Q \Phi$ & $\mathcal{A} \Phi \xi$ \\
\hline \begin{tabular}{|c|}
$\mathcal{A} T Q \Phi=Q+T+\mathcal{A} \Phi \xi \mathcal{B}$ \\
\\
$\mathcal{A} T Q \xi$
\end{tabular} & $\begin{array}{r} \\
\checkmark \\
\mathcal{A} \\
\mathcal{A} T Q\end{array}$ & $\begin{array}{l}\checkmark \\
\checkmark \\
\checkmark\end{array}$ & $\begin{array}{l}\checkmark \\
\checkmark \\
\checkmark\end{array}$ \\
\hline $\mathcal{A T Q} \Omega$ & \multicolumn{3}{|c|}{$\mathcal{A} T \xi \Omega=T+\mathcal{A} \xi \Omega \mathcal{B}|\mathcal{A} Q \xi \Omega=Q+A \xi \Omega \mathcal{B}| \mathcal{A} \Phi \xi \Omega=\Phi+\xi \Omega \mathcal{B}$} \\
\hline
\end{tabular}

TABLE II: Subclasses $\left(\mathcal{A}, Q_{i}, Q_{j}\right)$ of the five principal classes $\mathfrak{I}-\mathfrak{V}$ in Table $(\mathbb{I})$. The Table highlights the role of the zeros couples $(T Q)$ and and $\xi \Omega \mathcal{B}$.

\section{B. Analysis of the system stability, general conditions and results}

The symmetries of the system and the adapted $1+1+2$ formalism highlight the emergence of states certainly unstable for the linear (and non linear) perturbation. The unstable phases of the system with $\mathcal{A}=0$ are primarily regulated by the expansion (or the contraction) and the shear in the radial direction. These scalars are related by the sound velocity and the conductivity through a relation $\mathfrak{F}$ :

$$
\begin{aligned}
\mathfrak{F}: \quad & (\Sigma, \Theta) \mapsto\left(v_{s}, \sigma_{J}, \rho\right), \\
& \rho \mapsto\left(v_{s}, \sigma_{J}\right) .
\end{aligned}
$$

Conditions $\mathcal{C}$ in Eqs. 68 69:70) describe the system symmetries but not its stability, conversely the relations $\mathfrak{F}$ in Eqs. (71) relate the only kinematic variables of the radial shear and radial expansion, $\Sigma$ and $\Theta$ respectively, to the constants from the state and constitutive equations: the velocity of sound $v_{s}$ and the conductivity $\sigma_{J}$. We emphasize that Eq. (71) does not involves other dynamic variables such as the Weyl scalar or the electromagnetic contribution. Analogously for the five classes of solutions and their subclasses according to Eqs. 68 69 70 the relationship in (72) provides an upper bound on the matter density as a limiting value determined by the couple $\left(v_{s}, \sigma_{J}\right)$. The density, but not to its gradient, is in fact a variable in the system and, since this is and iso-entropic and barotropic fluid, one can use this to get information on the hydrostatic pressure the system is subjected to.

\begin{tabular}{|l|c|}
\hline $\mathfrak{C}$ & \\
\hline $\mathcal{C}(\mathcal{A}, T):$ & $\mathcal{E}+2 \Omega^{2}=p+\frac{\rho}{3}>0$ \\
$\mathcal{C}(\mathcal{A}, Q):$ & $E^{2}+B^{2}+2 \mathcal{E}=-(p+\rho / 3)<0$ \\
$\mathcal{C}(\mathcal{A}, \xi):$ & $\mathcal{B}=\Phi \Omega$ \\
$\mathcal{C}(\mathcal{A}, T, \Omega):$ & $\mathcal{E}=p+\rho / 3>0$ \\
$\mathcal{C}(\mathcal{A} \xi, \Omega):$ & $\mathcal{B}=0$ \\
\hline
\end{tabular}

TABLE III: Conditions $\mathfrak{C}$ as in Eq. 68) and Eq. 69. 


\begin{tabular}{|l|c|}
\hline Class & \\
\hline$(\mathcal{A}, T):$ & $\dot{\Omega}=0$ \\
$(\mathcal{A}, T, \xi):$ & $\dot{\Omega}=\dot{\Phi}=\dot{\mathcal{B}}=\dot{B}=0$ \\
$(\mathcal{A}, T, \Omega):$ & $\dot{\Phi}=0$ \\
\hline
\end{tabular}

TABLE IV: Condition $\mathfrak{C}$ in Eq. 70p. Classes of solutions and null evolution of the kinematic quantities.

\section{Discussion on the stability of the system with zero acceleration}

A relevant constraint $\mathfrak{F}$, for the stability of the system with null radial acceleration, is provided by condition (66) on the trace $\operatorname{Tr} \stackrel{\circ}{\mathbf{B}}$, and it can be expressed in terms of the expansion along the privileged direction as follows

$$
(\mathbf{u})_{\mathbf{0}}: \quad \Theta<\Theta_{u} \equiv \frac{6 \Sigma-\sigma_{J}}{\kappa}, \quad \kappa \equiv 7+v_{s}>1,
$$

this is a first condition for the instability of the system, providing an upper bound, a threshold for the occurrence of the instability, on the expansion or contraction with respect to the shear projected along the direction of symmetry i.e., the system is bound to be unstable if the expansion is, algebraically, smaller then $\Theta_{u}$, function of the radial shear, the sound velocity and the conductivity parameter. For small or zero conductivity the limit $\Theta_{u}$ is a fraction of the positive shear, where the system cannot contract or an instability occurs, for negative shear the contraction is limited by the value $\Theta_{u}<0$, for contractions too fast i.e. $\left.\Theta \in\right] \Theta_{u}, 0[$ the system is unstable according to Eq. (73). We can summarize the analysis in the as following points:

Systems in contraction $\Theta<0$ and in expansion $\Theta>0$ The expanding configurations, described the I and IV quadrant of Fig. (1), are favored in their stable phases with respect to the systems in contraction: the preference for the stable expansion is especially evident for negative shear, as in IV quadrant which describes also a part of the $(\mathcal{A}, Q)$ systems. In the fourth quadrant the stability conditions are always guaranteed within the condition $(73)$ as it is inf $\sigma_{J}>0$, the condition of the trace in Eq. $\sqrt{73}$ is not sufficient to establish the emergence of the instability for this system. Contrasting verses of the scalars $\Theta \Sigma<0$ appear to favor the equilibrium, this seems to be confirmed by the situation in the $\mathbf{I}$ quadrant, with positive shear, describing parts of the $(\mathcal{A}, T)$ systems. For slow expansions $\dot{\Theta} \approx 0$ and small conductivity, where the system is unstable, a threshold for the instability appears depending on the conductivity and can be easily seen by Eq. (73). Further restrictions on the symmetries of the system, in the subclasses of Table (IIII) will turn in a deformation of Eq. 733 as shown by Eq. (74). An increase of the shear corresponds in these cases to an increase of the instability regions in the plane $\Theta-\Sigma$ in Fig. (1). Considering the instability for the contracting systems, the II and III quadrants, we can see that the threshold for the emergence of the instability $\Theta_{u}$ decreases in general for positive shear up to the limiting value $\Sigma_{0} \equiv \sigma_{J} v_{s}$, the contraction is canceled and for higher shear the system begins an expanding phase, then the system will necessarily be unstable and the zone of stability will be limited to positive shear in the range $\Sigma \in] 0, \Sigma_{0}[$ and contraction $\Theta \in] \Theta_{0}, 0\left[\right.$, where $\Theta_{0} \equiv-\sigma_{J} / \kappa<0$ is the threshold for the stability of the systems in contraction with non-zero conductivity: for faster contractions $\Theta<\Theta_{0}$, the system with null shear is unstable. This region, however, increases with increasing conductivity, this means that the conductivity acts to stabilize the contracting configurations with positive shear. Systems $(\mathcal{A}, Q)$ are examples of configurations in the III quadrant: the systems are stable for high conductivity, negative shear and sufficiently low contractions, therefore the expanding $(\mathcal{A Q})$ systems are favored for the stability. In general for systems in contraction, in the II and III quadrants, a high conductivity is required for stable systems of $(\mathcal{A} \mathbf{T})$ and $(\mathcal{A} \mathbf{Q})$ classes respectively.

The role of the radial shear In the $\mathbf{I}$ and $\mathbf{I I}$ quadrants the radial shear is positive, an increases of $\Sigma$ generally acts to favor the instability of the configuration. An increase in magnitude of the negative shear in the III quadrant $(\Theta \Sigma>0)$, for contracting systems tends to favor the system stability for fast contractions.

The role of the conductivity In general an increase of the conductivity parameter particularly in the II and III quadrants, has a stabilizing effect on the system according to the law in Eq. (73). In the III quadrant, for example for systems of the $\mathfrak{I}$-class or $(\mathcal{A T})$ configurations, where the radial shear sign is equal to the contraction one, the stability region increases for low contractions and also for small conductivity as the shear is high enough in magnitude; ultimately for high conductivity the system can remain stable even for high radial contractions. The expansion phase is stable for negative shear, whereas at low expansion the system is unstable. The minimum threshold for the emergency of the instability increases with the shear but decreases with the conductivity, which has then a stabilizing effect on the system, for very high conductivity. The stable regions in the $\Theta-\Sigma$ plane increase by increasing the shear and therefore the stability is advantaged for high conductivity, for the 
expansion phase and for shear negative zero or small conductive. In particular the solutions of the IJI-class $(\mathcal{A Q})$ are a wider class of solutions admitting difference possible symmetries according to Table $(\mathrm{I})$, stable for any conductivity at negative shear, while for expanding systems as in the II quadrants the instability regions are maximum for smaller shear and zero for greater shear, the measure of this region increases with the conductivity. However the turning point of the expansion $\Theta$ is regulated by the $\sigma_{J} / \kappa$, for null radial shear $\Sigma$ it is $\Theta_{u}=-\sigma_{J} / \kappa$, the contraction is maximum as provided by $\Sigma_{x}$ and $\Theta_{x}$.

The role of the velocity of sound Fig. (2) emphasizes the role of the sound velocity in the determination of the unstable states of the system. The shear and expansion scalars have been normalized for the finite conductivity $\sigma_{J}$. For the systems included in the I-II and III quadrants, the velocity of sounds plays a significant role in the determination of the unstable phases, while there is no threshold for the emergence of the instability, crossing with continuity the III and IV quadrants of the $\Theta-\Sigma$ plane. In the limit of very large $v_{s}$ the configuration stops the expansion in the I quarter or the contraction phase in III one i.e. the threshold approaches $\Theta_{u}=0$. For approximately null velocity of sound it is $\Theta_{u} / \sigma_{J} \approx-1 / 7+(6 / 7) \Sigma$, then for sufficiently small $\Sigma$ the scalar expansion changes sign, and the expansion stage cancels the threshold for the instability, which instead appears for contracting systems. More specifically: there is a set for positive but small radial shear where the expansion threshold disappear, but for those shears the system is certainly unstable for sufficiently fast contractions. Generally the upper limit is small and the contracting system is always unstable: the expansion is null as the positive shear is $\Sigma / \sigma_{J}=1 / 6$, then for this configuration, the contracting phases are unstable. The solutions $\Theta=0$ and $\Sigma=0$ belongs to $(\mathcal{A}, \mathbf{T}, \mathbf{Q})$ class. For $\Sigma / \sigma_{J}>1 / 6$ the contracting system is always unstable for any couple $\left(\sigma_{J}, v_{s}\right)$. Decreasing the velocity of the sound, the region of $\Theta-\Sigma$ plane, for expanding systems correspond to unstable regions for an increase of the positive shear. As the velocity of sound decreases for positive shear the instability region increases. The instability in the $\mathbf{I}$ quadrant is carried out for sufficiently high expansions and shear. The increase of the speed of sound acts to stabilize the expanding system for positive shear and destabilize the contracting systems at $\Sigma / \sigma_{J}>1 / 6$, in the window of smaller but positive shear, the increase of the speed of sound acts to destabilize the system while a decrease of this corresponds to an increase the stability provided that the contraction is sufficiently small in magnitude or $\Theta / \sigma_{J}>-1 /\left(v_{s}+7\right)$.

We now focus on the effects the velocity of sound in the case of negative shear: for the expanding system in the IV quadrant, no threshold exists. For contracting systems in the III quadrant, an increase of the speed of sound increases the stability of the system increasing in magnitude the threshold for very high contractions. A decrease of the velocity of sound, for contracting systems with negative shear, induces the system unstable even for very small expansions. The increase in magnitude of the radial shear increases the system stability. Ultimately the system's equilibrium depends on the velocity of sound in I and III quadrants, that is for concordant shear and expansion or in the $\mathbf{I V}$ quadrant for systems in contraction with positive shear smaller than $1 / 6 \sigma_{J}$. In the limit of very large velocity of sound $\Theta_{u}>0$ for $\Sigma / \sigma_{J}>1 / 6$, for $\left.\Sigma / \sigma_{J} \in\right]-\infty, 1 / 6\left[\right.$ it is $\Theta_{u}<0$.

\section{Stability of the subclasses with restricted symmetries}

Additional restrictions on the system with $\Sigma \neq 0$ and $\Theta \neq 0$, and therefore on the perturbations modify the condition 73 on the reduced system as follows

$$
\Sigma \geq\left.\Sigma_{0}\right|_{\mathcal{C}},\left.\quad \Sigma_{0}\right|_{\mathcal{C}} \equiv a\left[\left(b+c v_{s}\right) \Theta+c \sigma_{J}\right], \quad a<1 \quad \text { or } \quad a \ll 1, \quad b \gg c, \quad c \geq 1, \quad\{a, b, c\} \in \mathbb{N},
$$

the quantities $\{a, b, c\}$ change depending on the class of solutions. In terms of the expansion $\Theta$, analogously to Eq. (73) one has

$$
\Theta \leq\left.\Theta_{0}\right|_{\mathcal{C}},\left.\quad \Theta_{0}\right|_{\mathcal{C}} \equiv \frac{\Sigma-a c \sigma_{J}}{a\left(b+c v_{s}\right)} .
$$

then the expansion limit is null $\left.\Theta_{0}\right|_{\mathcal{C}}=0$. For $\Sigma_{\max } \equiv a c \sigma_{J}$, for smaller shear $\Sigma<\Sigma_{\max }$ the expanding systems are always stables while a threshold for the instability of the contracting systems appears: for $\Theta<\left.\Theta_{0}\right|_{\mathcal{C}}$ the system is unstable. It is noteworthy that the shear limit $\left.\Sigma_{0}\right|_{\mathcal{C}}=0$ is cancelled for maximal contractions equal to $\Theta_{\max } \equiv$ $-c \sigma_{J} /\left(b+c v_{s}\right)$. More generally, for each subclasses of Table (II) and Table (II) the unstable phase will be regulated by some limiting extreme values (LEV) for the systems $\Theta=0$ or $\Sigma=0$ providing the boundary values for the regions 


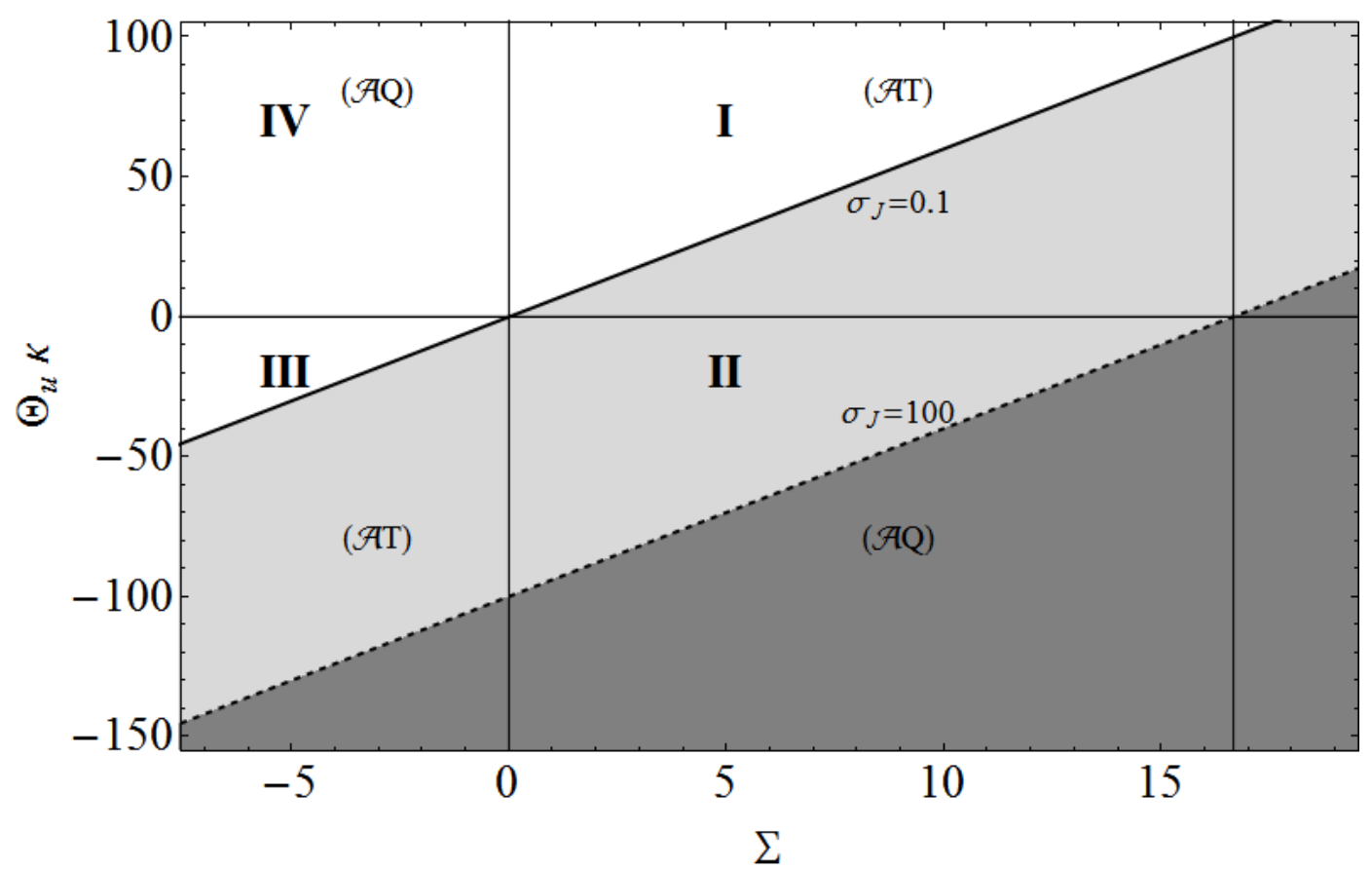

FIG. 1: Plot of the limiting expansion $\Theta_{u} \kappa$, defined in Eq. 73 as function of the shear in the preferred radial direction where $\kappa \equiv v_{s}+7$. per $\sigma_{J}=0.1$ (black line ) e $\sigma_{J}=100$ (dashed black line Possibly an identical plot for the expansion $\Theta_{u}$ in terms of $\sigma_{J} / \kappa$ and $\Sigma \kappa$. The instability regions, for $\Theta<\Theta_{u}$, are colored: then for $\sigma_{J}=0.1$ the gray region, and light gray region determines the instability for configurations with conductivity parameter $\sigma_{J}=100$, gray region is then stable for this system. In the white region the system can be stable. Configurations $(\mathcal{A T})$ are in the I and III quadrants $(\Sigma \Theta>0)$ as in the $T=0$ class of solution, for $\Sigma=(2 / 3) \Theta$, while the $(\mathcal{A}$, Q $)$ class for $\Sigma=-(1 / 3) \Theta$ belongs to the II and IV quadrants $(\Sigma \Theta<0)$. The stability of a system expanding along the direction of symmetry (but not accelerating, as it is $\mathcal{A}=0$ ) is regulated as in the $\mathbf{I}$ and IV quadrants. The contraction in II and III quadrants, where the radial shear is positive and negative, respectively. The classes are summarized in Table (I). The contraction limit vanishes, and the stability limit is only on the radial expansion for high enough shear according to $\Sigma=\sigma_{J} / 6$.

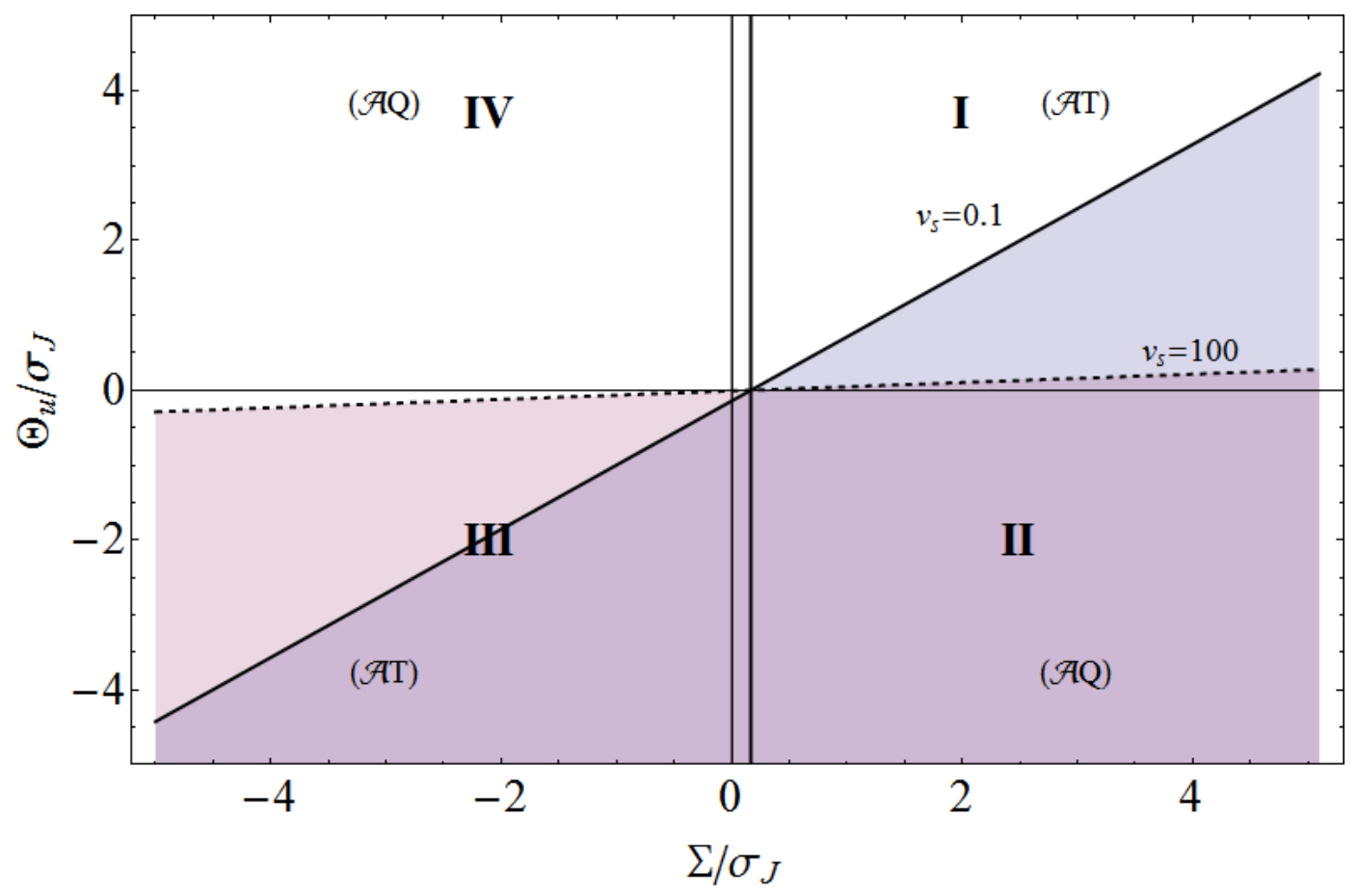

FIG. 2: Plot of the limiting expansion $\Theta_{u} / \sigma_{J}$, in Eq. 73), versus the radial shear $\Sigma / \sigma_{J}$ for $v_{s}=0.01$ (black line) and $v_{s}=100$ (dashed black line). The Shaded regions marke the sections in the $\Theta-\Sigma$ plane where instability occurs as $\Theta<\Theta_{u}$. It is $\Theta_{u}=0$ for $\Sigma / \sigma_{J}=1 / 6$. 

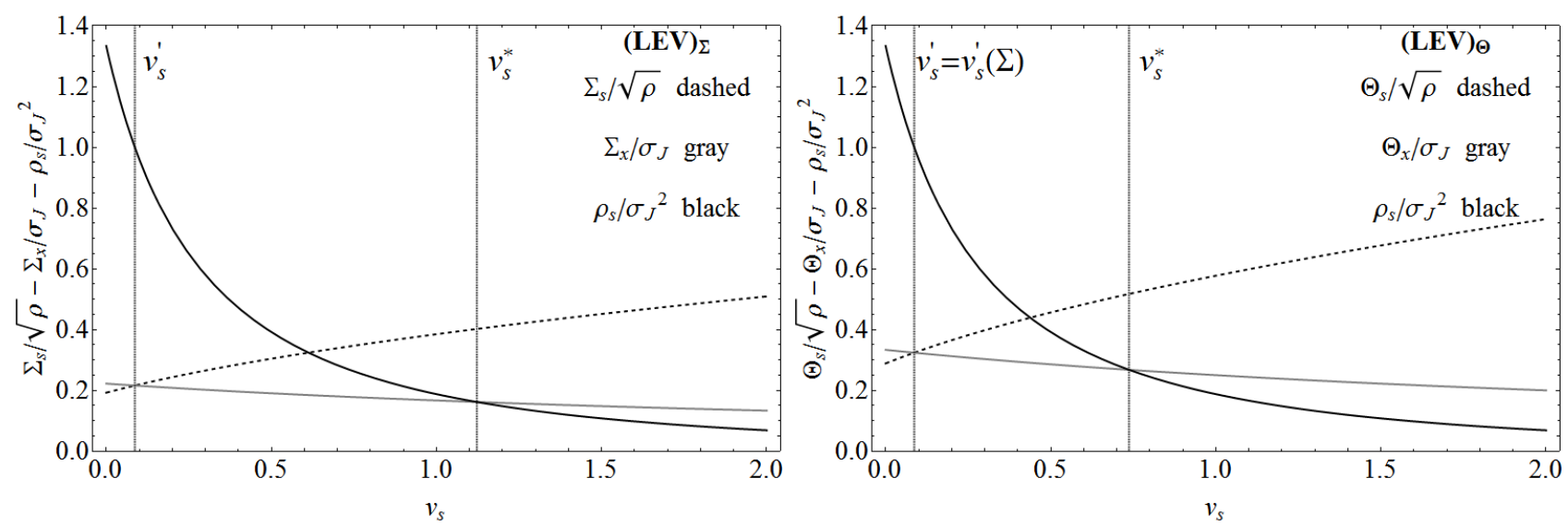

FIG. 3: Ratios of the limiting extreme values $(\mathbf{L E V})_{\Sigma}$ (left panel) and (LEV) $\Theta$ introduced in Eq. 76 , functions of the velocity of sound $v_{s}$. At at $v_{s}^{*}$ it is $\Sigma_{x}=\sigma_{J}^{-1} \rho_{s}=\frac{2 \sigma_{J}}{4+\sqrt{70}} \approx 0.16 \sigma_{J}$ while $\Theta_{x}=0.267592 \sigma_{J}$, in $v_{s}^{\prime}$ it is $\Sigma_{s}=\Sigma_{x} \sqrt{\rho} / \sigma_{J} \approx 0.22 \sqrt{\rho}$ analogously $\Theta_{s}=0.27 \sqrt{\rho}$, in the cross point in $] v_{s}^{\prime}, v_{s}^{*}\left[\right.$ it is $\rho_{s}=0.32 \sigma_{J}^{2}$ where $\Sigma_{s}=0.32 \sqrt{\rho}$ in $(\mathbf{L E V})_{\boldsymbol{\Sigma}}$ while the ratio is $\rho_{s} / \sigma_{J}^{2} \approx 0.44$ in $(\mathbf{L E V})_{\Theta}$.

of the plane $\Theta-\Sigma$ respectively for the stability of the system:

$$
\begin{array}{ll}
(\mathbf{L E V})_{\Sigma}: & \rho_{s} \equiv \frac{12 \sigma_{J}^{2}}{\left(3+v_{s}\right)^{2}\left(1+3 v_{s}\right)}, \quad \Sigma_{s} \equiv \frac{1}{3} \sqrt{\left(\frac{1}{3}+v_{s}\right)} \rho, \quad \Sigma_{x} \equiv \frac{2 \sigma_{J}}{3\left(3+v_{s}\right)} . \\
(\mathbf{L E V})_{\Theta}: & \rho_{s}=\left.\rho_{s}\right|_{(\mathbf{L E V})_{\Sigma}}, \quad \Theta_{s} \equiv \frac{3}{2} \Sigma_{s}, \quad \Theta_{x} \equiv \frac{3}{2} \Sigma_{x} .
\end{array}
$$

see Fig. (3). A threshold exists for the stability in the systems with the density in the two regimes $\rho>\rho_{s}$ and $\rho<\rho_{s}$, according to $(\mathbf{L E V})_{\Sigma}$ or $(\mathbf{L E V})_{\Theta}$, with conditions on the radial shear or respectively the expansion or contraction. The details of the analysis are considered in Sec. (D). For the stability in general one has the two following cases:

$$
\begin{array}{ll}
\rho<\rho_{s} & \left.\Delta \in\left[-\Delta_{x},-\Delta_{s}\right] \text { or }\right]+\Delta_{s},+\infty[ \\
\rho>\rho_{s} & ]-\Delta_{s}, \Delta_{s}[\text { or }]+\Delta_{s},+\infty\left[, \quad \Delta=\Sigma \text { for } \quad(\mathbf{L E V})_{\Sigma}, \quad \Delta=\Theta \quad \text { for } \quad(\mathbf{L E V})_{\Theta}\right.
\end{array}
$$

each of the two regions are typically regulated by additional conditions on $Q_{i} \in \vec{\nu}(\mathbf{L E V})_{\Sigma}$, for example for the $\mathfrak{I}$-class, $(\mathcal{A T})$ solution, are determined by a set of conditions on $\mathcal{B}, \xi$ and $E$. We note that the limiting value on the density $\rho_{s}$, increases with the conductivity but decreases with the velocity of sound: there are three ranges of $v_{s}$ to be considered as detailed in Fig. (3). However the boundary $\Delta_{s}$ depends on the density $\rho$ and therefore differently regulated in the three regions. For low density regimes $\rho<\rho_{s}$, the density function regulates the system stability in a reduced region of the $\rho-\Sigma$ plane through the conditions imposed on shear or the expansion especially for low values. This situation is more evident for low velocity of sounds $v<v_{s}^{*}$. For larger values the ranges for low density variations are restricted to $\rho<0.2 \sigma_{J}$.

\section{Comments on the five principal classes of solutions}

$\mathfrak{I}$-Class $(\mathcal{A} \mathbf{T})$ : this case is constrained by the condition $\mathcal{C}(\mathcal{A}, T)$ obtained from Eq. 60a. This relation is a consequence of the condition $T=0$, and it will occur in the other subcases characterised by this assumption. The evolution equation for the parallel vorticity $\Omega$ is $\dot{\Omega}=0$ : this quantity remains constant along the fluid motion, for the perturbed quantity as well as the unperturbed one. Furthermore, the time evolution of the matter density only involves the kinematic variable $Q$. We can draw some conclusions on the stability of the system on the basis of the matrix trace. The system is linearly unstable if with a negative shear on the radial direction which is bounded by $\Sigma^{\circ} \leq \Sigma_{x}$. It is worth noting that the limiting case is defined only by the constitutive equation, the conductivity $\sigma_{J}$, and the equation of state by $v_{s}$ - fixed by the reference solution. Furthermore, considering the coefficients $c_{9}=-1$ and $c_{8}=\operatorname{Tr} \stackrel{\circ}{\mathbf{B}}<0$ we infer the condition for the stability $c_{1}<0$. Using the condition $\mathcal{C}(\mathcal{A}, T)$, this condition can be rephrased as the following two alternatives: (i) $\stackrel{\circ}{\rho}<\stackrel{\circ}{\rho}_{s}$ with $\stackrel{\circ}{\Sigma} \in\left(-\stackrel{\circ}{\Sigma}_{x},-\stackrel{\circ}{\Sigma}_{s}\right) \cup\left(\stackrel{\circ}{\Sigma}_{s},+\infty\right)$ and (ii) $\stackrel{\circ}{\rho} \geq \stackrel{\circ}{\rho}_{s}$ with $\stackrel{\circ}{\Sigma}^{\prime}\left(\stackrel{\circ}{\Sigma}_{s},+\infty\right)$, where the $(\mathbf{L E V})_{\Sigma}$ hold. 
II-Class $(\mathcal{A} \mathbf{Q})$ : the condition $\frac{1}{3} \Theta=-\Sigma$ implies that the matter and field variables are related by the constraint $\mathcal{C}(\mathcal{A}, Q)$-see equation $(60 \mathrm{~b})$. This relation is a consequence of the assumption $Q=0$, and it will occur in the other subcases characterised by this hypothesis. We observe that the assumption $Q=0$ implies in particular that the expansion of the 3-sheets and the radial component of the shear of the 3-sheet must have opposite sign. Studying the problem for the reduced system of nine variables we find that the system is linearly unstable if $\operatorname{Tr} \stackrel{o}{\mathbf{B}}>0$ - that is if $\stackrel{\circ}{\mathrm{i}}$ is negative and bounded above by $\stackrel{\circ}{\mathrm{O}} \leq\left.\Theta_{0}\right|_{\mathcal{C}}$ with $(a=c=1 ; b=9)$. Again, as in the case $T=0$, we obtain a constraint for the radial expansion that depends on the unperturbed constants $\left(\sigma_{J}, v_{s}\right)$.

III-Class $(\mathcal{A} \Phi)$ : the assumptions $\mathcal{A}=0$ and $\Phi=0$ on equations $(\sqrt{59 f})$ and $(\sqrt{59 g})$ give rise to two possible subcases: (i) $\Omega=0$ or (ii) $\xi=0$ and $\mathcal{B}=0$. We consider therefore these two subcases separately:

$(\mathcal{A} \Phi \boldsymbol{\Omega})$ : the trace implies the following condition of instability $\stackrel{\circ}{\Sigma} \geq\left.\Sigma_{0}\right|_{\mathcal{C}}$ with $(a=2 / 9, b=6, c=1)$. We note that for the reference solution $\Theta$ and $\stackrel{\circ}{\Sigma}$ are related by the square of the velocity of sound and the conductivity through $\sigma_{J}$. However, the sign of the $\Sigma \Theta$ is not constrained by this relation.

$(\mathcal{A} \Phi \xi \mathcal{B})$ : in this case $T$ is the only kinematical variable involved in the evolution of the electromagnetic field.

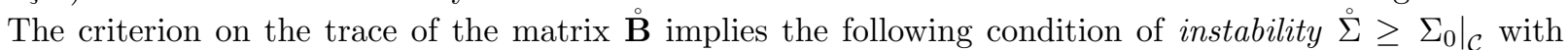
$(a=2 / 21, b=16, c=3)$.

Thus, we finally note that the subcases (i) and (ii) are characterised by similar constraints on $\stackrel{\circ}{\Sigma}$ and $\Theta$.

IV - Class $(\mathcal{A} \xi)$ : equation $\sqrt{59 g}$ leads to the condition $\mathcal{C}(\mathcal{A}, \xi):$. On the other hand the condition of instability on the trace of the matrix $\mathbf{B}$ leads to the inequality $\stackrel{\circ}{\Sigma} \geq\left.\Sigma_{0}\right|_{\mathcal{C}}$ with $(a=1 / 33, b=40, c=6)$.

$\mathfrak{V}-$ Class $(\mathcal{A} \boldsymbol{\Omega})$ : in this case the system is unstable if $\stackrel{\circ}{\Sigma} \geq\left.\Sigma_{0}\right|_{\mathcal{C}}$ with $(a=1 / 15, b=19, c=3)$.

We conclude this Section pointing out that the stability of the configuration under consideration is constrained by similar relations between the unperturbed radial part of the shear of the 3 -sheet $\Sigma^{\circ}$ and the radial expansion $\Theta_{\Theta}$. These constraints only depend on the square of the sound velocity and the conductivity. In Sec. (D) we specialise this analysis to consider the subclasses of Tables (II) and (II).

\section{CONCLUSIONS}

In this article we have explored the stability proprieties of an ideal, LRS Einstein-Maxwell perfect fluid system. As a first step we have formulated an hyperbolic initial value problem providing a suitable (quasilinear) symmetric hyperbolic system by means of which one can address the nonlinear stability of this system. This first result allows us to provide a suitable formulation of an initial value problem, that is a necessary issue for the construction of the numerical solutions, ensuring the local and global existence problems. Moreover the problem of the propagation of the constraints can be assumed satisfied at all time and then prove this result by fairly general arguments as discussed in 23, 24. Our analysis is based on a 1+1+2-tetrad formalism. In our calculations certain choices of kinematic properties of the configuration were motivated by the necessity to extract some information from the rather complicated equations governing the perturbations. We studied five principal classes of solutions and different subcases, considering systems with particular kinematic configurations. The assumption of the radial symmetry simplifies the problem and takes great advantage from the $1+1+2$-decomposition [25]. Thus, in this work we have proceeded as follows: we wrote the $1+1+2$-equations for the LRS system using the radial vector, pointing along the axis of symmetry. Then, a discussion on the thermodynamical quantities of the system was been provided. For the linear perturbation analysis it was useful to introduce a re-parametrised set of evolution equations based of a suitable combination of the radially projected shear and expansion. The resulting evolution system was then used to analyse the stability problem for small linear perturbations of the background. We presented the re-parameterized set of evolution equations collected in a proper symmetric hyperbolic form and discussed the perturbation to the first order of the variables. We have presented the main results concerning the linear stability of this system in a classification of subcases that constitutes the principal result of this paper. In the Appendix to this article, we provide also an alterative symmetric hyperbolic system for the fluid fields within the $1+1+2$-decomposition followed by some general notes on the evolution equations and hyperbolicity considerations. A suitable propagation equation for the fluid radial acceleration is there recovered by the introduction of a new unknown field corresponding to the derivative of the matter density projected along the radial direction. suitable field and evolution equations can be obtained for this quantity. The set of evolution equations is complemented by the constraint and constitutive equations. Restricting our attention to isotropic fluids (entropy 
is a constant of both space and time) we considered a one species particle fluid (simple fluid) and we introduced a polytropic equation of state with a constant velocity of sound. In order to close the system of evolution equations it is necessary to specify the form of the conduction current. Accordingly, we assumed the Ohm's law so that a linear relation between the conduction current and the electric field, involving a constant electrical conductivity coefficient, holds. We have assumed that the pressure of the fluid $p$ and the charge density $\varrho_{C}$ to be functions of the matter density $\rho$, and the charge current $j_{c}$ function of the electric field $E$. Although viable, the perturbed equation for the radially projected acceleration $\mathcal{A}$ turned out to be a very complicated expression of the other variables and their derivatives. Thus, in order proceed in the stability analysis, we studied a simplified form system by taking up some assumptions on the configuration. Assuming a null radial acceleration for the reference solution, our analysis is particularly focused on some specific cases defined by fixing the expansions of the 3-sheets and 2-sheets, the radial part of the shear of the 3-sheet, the twisting of the 2-sheet and the radial part of the vorticity of the 3-sheet. In this way we are also able to provide results concerning the structure of the associated LSS taking into considerations all the different subcases. This analysis constitutes the main result of the paper. In particular, we found that in many cases the stability conditions can be strongly determined by the constitutive equations by means of the square of the velocity of sound and the electric conductivity. In particular, this is evident for the contracting and expanding LRS configurations: a threshold for the emergence of the instability appears in both cases. The conditions bind mainly the expansion (viceversa contraction) along the preferred direction with respect to different regimes of the radial shears. These results provide in a quite immediate manner information regarding were certainly unstable system. The results for this type of configurations are illustrated in Sec. (VIIB) and schematically in Fig. (1) and Fig. (2), in the four fundamental cases emphasizing the role of the couple of parameters $\left(v_{s}, \sigma_{J}\right)$ moreover together with these parameters the relative sign of scalars $\Sigma$ and $\Theta$ plays an essential role in the determination of the unstable phases of the systems. For expanding configurations with $\Sigma \Theta<0$, appears no threshold for the emergence of instability by means of condition (66), this does not mean that expanding systems with negative sher are in any condition certainly stable, but further conditions can be provided as we discussed in dealing with different classes of solutions. The interesting situation is in the remaining three cases, the role of the velocity of sound and the conductivity acts in a different way for the systems in the three regions of the Figs. (12), according to our analysis to favor or not certainly unstable states of the system; in any case there is always is a specific threshold for the contraction or expansion, above which in the first case for contracting systems with a fast contraction rate $|\Theta|>\left|\Theta_{u}\left(\sigma_{J}, v_{s}\right)\right|>0$ or expansions at $0<\Theta<\Theta_{u}\left(\sigma_{J}, v_{s}\right)$ system is certainly unstable. The other cases and subcases show similar situations where the threshold provided by the density values, and the couple $(\Sigma, \Theta)$ varies in in different ranges depending on $\left(\rho, v_{s}, \sigma_{J}\right)$. The magnetic field does not have a specific role in determining the stability of the system, and the Maxwell field and the geometrical effects enclosed by the magnetic and electric part of the Weyl tensor.

Concerning the methods, the core of the stability analysis is given by the study of the non principal part of the matrix $\stackrel{\circ}{\mathbf{B}}$ of the system using some relaxed stability eigenvalue conditions. However, a full analysis of the stability properties of the system turns extremely cumbersome because of the form of the matrix. Thus, we proceeded with the analysis of the eigenvalues using an indirect method aimed at determining to know the sign of these. Repeatedly use the fact that a sufficient condition for the instability of the system is the existence of at last one positive eigenvalues. This simple requirement is nevertheless able to provide a immediate way to show the conditions where the linear instability occurs. In several places we made use of the Descartes criterion to determine the maximum number of positive and negative real roots of the characteristic polynomial. In particularly simple cases one can make use of the so-called Routh-Hurwitz criterion to determine the number of roots with positive and negative real part of the polynomial by constructing the Routh associated matrix. It may be possible to use some of these criteria for some specific cases and we expect future work to include a development of this paper in this direction.

\section{Acknowledgments}

DP gratefully acknowledges support from the Blanceflor Boncompagni-Ludovisi, née Bildt and wishes to thank the Angelo Della Riccia Foundation and thank the institutional support of the Faculty of Philosophy and Science of the Silesian University of Opava.

\section{Appendix A: A symmetric hyperbolic system for the fluid fields}

In this Section we provide a brief discussion of a construction leading to a suitable propagation equation for the radial acceleration $\mathcal{A}$. In addition, we also provide an alternative set of the propagation equations for the other kinematic and field variables. A similar construction has been given in a slightly different context in [40. 
For the sake of convenience let us define $g_{a b} u^{a} u^{b}=u_{0} u^{0}+u^{i} u_{i}=\epsilon$. In the present case $\epsilon=-1$. Using the identity $u_{a} \nabla_{b} u^{a}=0$ we readily conclude that

$$
\nabla_{a} u^{0}=-\frac{u_{i}}{u_{0}} \nabla_{a} u^{i}
$$

Moreover, one has that $\nabla_{c} \nabla_{d} u^{0}+\nabla_{c} \nabla_{d} u^{i}=-\frac{1}{u_{0}} \nabla_{c} u_{i} \nabla_{d} u^{i}-\frac{u_{i} u^{j}}{\left(u_{0}\right)^{2} u^{0}} \nabla_{d} u^{i} \nabla_{c} u_{j}$. From the Bianchi identity and the inhomogeneous Maxwell equation we have that

$$
\nabla^{a} F_{a c}=+\varepsilon J_{c}
$$

In this last equation we introduce $\varepsilon=-1$, to match equations A2 and (21). In addition, we have the continuity equation for the matter density $\rho$

$$
u_{a} \nabla^{a} \rho+(p+\rho) \nabla^{a} u_{a}-\varepsilon u^{b} F_{b}^{c} J_{c}=0,
$$

and the Euler equation

$$
(p+\rho) u^{a} \nabla_{a} u^{c}-\epsilon h^{b c} \nabla_{b} p-\varepsilon \epsilon J_{b} F^{c b}=0 .
$$

This last equation can be alternatively written as

$$
(p+\rho) u^{a} \nabla_{a} u^{c}-\epsilon \nabla^{c} p+u^{c} u^{b} \nabla_{b} p-\varepsilon \epsilon J_{b} F^{c b}=0 .
$$

In what follows, we will consider a barotropic equation of state $p=p(\rho)$ and introduce here the quantity $v_{s}$ defined by the relation $\nabla_{b} p=\left(\frac{\partial p}{\partial \rho}\right) \nabla_{b} \rho=v_{s} \nabla_{b} \rho$. Note that $v_{s}=\nu_{s}^{2}$, where $\nu_{s}$ is the speed of sound introduced in equation 49. Thus Eq. A5 is now

$$
(p+\rho) u^{a} \nabla_{a} u^{c}-\epsilon v_{s} \nabla^{c} \rho+v_{s} u^{c} u^{b} \nabla_{b} \rho-\varepsilon \epsilon J_{b} F^{c b}=0 .
$$

Using equations $\mathrm{A} 3$ and $\mathrm{A} 6$, we obtain:

$$
\nabla_{c} \rho=\epsilon \frac{(\rho+p)}{v_{s}} u^{a} \nabla_{a} u_{c}-\epsilon(p+\rho)\left(\nabla_{a} u^{a}\right) u_{c}-\varepsilon \frac{J^{b} F_{c b}}{v_{s}}+\varepsilon \epsilon u^{b} F_{b d} J^{d} u_{c} .
$$

Using again the normalisation condition we can write $u_{0}$ as: $u_{0}=\epsilon u^{0}=\beta \sqrt{\epsilon\left(\epsilon-u_{i} u^{i}\right)}$, where $g^{00}=\eta^{00}=\epsilon$ and $\beta=\beta(\epsilon)$ is a sign to be fixed according to the metric signature conventions.

Now, using equations A1 in equation A3 we find

$$
\left(u^{0} \nabla_{0} \rho+u^{i} \nabla_{i} \rho\right)+(\rho+p)\left(\nabla_{i} u^{i}-\frac{u_{i}}{u_{0}} \nabla_{0} u^{i}\right)-\varepsilon u^{b} F_{b}^{c} J_{c}=0
$$

and from equation $\mathrm{A} 6$

$$
\begin{aligned}
& \tilde{\mathcal{E}}^{(0)} \equiv u^{a} \nabla_{a} u^{0}-\frac{\epsilon v_{s}}{\rho+p}\left(\nabla_{c} g^{c 0}\right)+\frac{u^{0} v_{s}}{\rho+p} u^{b} \nabla_{b} \rho-\varepsilon \epsilon J_{b} F^{0 b}=0 \\
& \tilde{\mathcal{E}}^{(i)} \equiv u^{a} \nabla_{a} u^{i}-\frac{\epsilon v_{s}}{\rho+p}\left(\nabla_{c} g^{c i}\right)+\frac{u^{i} v_{s}}{\rho+p} u^{b} \nabla_{b} \rho-\varepsilon \epsilon J_{b} F^{i b}=0
\end{aligned}
$$

Thus, introducing the zero-quantity:

$$
\varsigma^{i} \equiv \frac{u^{i}}{u^{0}} \tilde{\mathcal{E}}^{(0)}-\tilde{\mathcal{E}}^{(i)}=0
$$

we obtain the equation

$$
\varsigma^{i}=-\left(u^{a} \nabla_{a} u^{i}+\frac{u^{i} u_{j}}{u^{0} u_{0}} u^{a} \nabla_{a} u^{j}\right)+\frac{\epsilon v_{s}}{\rho+p} \nabla_{c} \rho\left(g^{c i}-g^{c 0} \frac{u^{i}}{u^{0}}\right)+\epsilon \varepsilon J_{b}\left(F^{i b}-\frac{u^{i}}{u^{0}} F^{0 b}\right)=0 .
$$

Equations A8, A9 ) constitute, after multiplication by a suitable numerical factor, an hyperbolic system for $\rho$ and $u^{i}$. 
In what follows, It turns to be necessary to introduce here the fields

$$
\mu_{a} \equiv \nabla_{a} \rho, \quad U_{a b} \equiv \nabla_{a} u_{b}
$$

where $\nabla_{[a} \mu_{b]}=0$. One readily can verify that $U_{a b} u^{b}=0, \quad U_{a 0}=-\frac{u^{i}}{u^{0}} U_{a i}$, and that

$$
\begin{aligned}
& \nabla_{a} U_{b}^{0}=-\frac{u_{i}}{u_{0}} \nabla_{a} U_{b}^{i}-\frac{U_{a}^{0}}{u_{0}} U_{b 0}-\frac{U_{a}^{i}}{u_{0}} U_{b i} \\
& u^{b} \nabla_{a} U_{c b}=-U_{c b} U_{a}{ }^{b} .
\end{aligned}
$$

Now, applying a covariant derivative to the equations of motion of the fluid and commuting gives

$$
Z_{c b} \equiv\left(1+v_{s}\right) u_{b} u^{a} \nabla_{a} \mu_{c}+(\rho+p)\left(u_{b} \nabla_{a} U_{c}{ }^{a}+u^{a} \nabla_{a} U_{c b}\right)-\epsilon v_{s} \nabla_{c} \mu_{b}+W_{c b}=0
$$

where

$$
\begin{gathered}
W_{c b} \equiv(\rho+p)\left(R_{d c a}^{a} u^{d} u_{b}+R_{c a b d} u^{d} u^{a}\right)+U_{c b} u^{a} \mu_{a}\left(1+v_{s}\right)+u_{b} U_{c}{ }^{a} \mu_{a}\left(1+v_{s}\right)+\mu_{c}\left(1+v_{s}\right)\left(u_{b} U_{a}^{a}+u^{a} U_{a b}\right) \\
+(\rho+p)\left(U_{c b} U_{a}^{a}+U_{c}{ }^{a} U_{a b}\right)-\epsilon \nabla_{c} v_{s} \mu_{b}-\epsilon \nabla_{c}\left(J_{a} F_{b}{ }^{a}\right)+u_{b} \mu_{c} u^{a} \nabla_{a} v_{s}
\end{gathered}
$$

Using equation A12 we obtain

$$
u^{b} Z_{c b}=\epsilon u^{a} \nabla_{a} \mu_{c}+\epsilon(\rho+p) \nabla_{a} U_{c}^{a}+X_{c},
$$

with $X_{c} \equiv u^{b} W_{c b}-(\rho+p) u^{a} U_{c b} U_{a}^{b}$, where

$$
u^{b} W_{c b}=\epsilon\left((\rho+p) R_{d c a}^{a} u^{d}+\mu_{a} U_{c}^{a}\left(1+v_{s}\right)+\mu_{c}\left(1+v_{s}\right) U_{a}^{a}-\mu_{b} u^{b} \nabla_{c} v_{s}-u^{b} \nabla_{c}\left(J_{a} F_{b}^{a}\right)+\mu_{c} u^{a} \nabla_{a} v_{s}\right) .
$$

In addition, one has that $h_{d}^{b} Z_{c b}=(\rho+p) u^{a} \nabla_{a} U_{c d}-\epsilon v_{s} \nabla_{c} \mu_{d}+v_{s} u_{d} u^{b} \nabla_{b} \mu_{c}+Y_{c d}=0$, with $Y_{c d}=h_{d}^{b} W_{c b}+\epsilon(\rho+$ p) $u_{d} U_{c b} U_{a}{ }^{b} u^{a}$.

Equation A14 can be written as

$$
\epsilon\left[\left(u^{0} \nabla_{0} \mu_{c}+u^{i} \nabla_{i} \mu_{c}\right)+\epsilon(\rho+p)\left(\nabla_{i} U_{c}^{i}-\frac{u_{i}}{u_{0}} \nabla_{0} U_{c}^{i}\right)+\hat{X}_{c}=0\right.
$$

with

$$
\hat{X}_{c} \equiv X_{c}-\frac{1}{u_{0}}\left(U_{0}{ }^{a} U_{c a}\right)
$$

Now, the combination $\left(u^{i} / u_{0}\right) h^{0 b} Z_{c b}-h^{i b} Z_{c b}$, leads to the equation

$$
\begin{gathered}
-(\rho+p)\left(u^{a} \nabla_{a} U_{c}^{i}+\frac{u^{i}}{\left(u_{0}\right)^{2}} u_{j} u^{a} \nabla_{a} U_{c}{ }^{j}\right)+\epsilon v_{s}\left(g^{d i} \nabla_{d} \mu_{c}-\frac{u^{i}}{u_{0}} g^{d 0} \nabla_{d} \mu_{c}\right)+\left(\frac{u^{i}}{u_{0}} g^{d 0}-g^{i d}\right) Y_{c d} \\
-(\rho+p) \frac{u^{i}}{\left(u_{0}\right)^{2}} u^{a} U_{a}^{b} U_{c b}+v_{s} u^{i} u^{b} \nabla_{b} \mu_{c}(\epsilon-1)=0
\end{gathered}
$$

Viceversa, the combination $\left(u^{i} / u^{0}\right) h^{0 b} Z_{c b}-h^{i b} Z_{c b}$ leads to the equation

$$
(\rho+p)\left(u^{a} \nabla_{a} U_{c}^{i}+\frac{u^{i} u_{j} u^{a} \nabla_{a} U_{c}^{j}}{u_{0} u^{0}}\right)-\epsilon v_{s}\left(g^{d i} \nabla_{d} \mu_{c}-\frac{u^{i}}{u^{0}}\left(\nabla_{d} \mu_{c}\right) g^{d 0}\right)+\hat{Y}_{c}^{i}=0
$$

where

$$
\hat{Y}_{c}^{i} \equiv(\rho+p) \frac{u^{a} u^{i}}{u_{0} u^{0}} U_{a}^{b} U_{c b}-\left(g^{d 0} \frac{u^{i}}{u^{0}}-g^{d i}\right) Y_{c d}
$$

Equations A15 and A19 constitute a symmetric hyperbolic system for the fields $\mu_{a}$ and $U_{a}{ }^{i}$. 


\section{Appendix B: $1+1+2$-decomposition}

In this Section we study the $1+1+2$-decomposition of the term $Z_{c b}$ in equation A13). In what follows we fix $\epsilon=-1$. Recall that up to now $\left(\rho, \mu_{c}, u^{i}, U_{a b}\right)$ have been used as independent variables for our evolution system. We now consider $\left(\rho, \mu_{c}, u^{i}\right)$ and the quantities $\left(\| \mathcal{A},{ }^{\perp} \mathcal{A}^{a}, \Theta, \Sigma, \Omega,{ }^{\perp} \Sigma_{a},{ }^{\perp} \Omega^{a},{ }^{\perp} \Sigma_{a}^{b}\right)$ as independent variables, the last being defined by the decomposition of the $U_{a b}$. This decomposition is obtained from considering " $\mathcal{A}=u^{b} n^{a} U_{b a}$, and $U_{a b}=-u_{a} n_{b} \| \mathcal{A}+\widetilde{U}_{a b}$, where

$$
\widetilde{U}_{a b} \equiv+n_{a} n_{b}\left(\frac{1}{2} \| \Theta+{ }^{\|} \Sigma\right)+N_{a b}\left(\frac{1}{3}\left\|\Theta-\frac{1}{2}\right\| \Sigma\right)+{ }^{\|} \Omega \epsilon_{a b}+{ }^{\perp} \widetilde{U}_{a b}
$$

with

$$
{ }^{\perp} \widetilde{U}_{a b} \equiv-u a^{\perp} \mathcal{A}_{b}+n_{a}\left({ }^{\perp} \Sigma_{b}+\epsilon_{b c}{ }^{\perp} \Omega^{c}\right)+\left({ }^{\perp} \Sigma_{a}-\epsilon_{a c}{ }^{\perp} \Omega^{c}\right) n_{b}+{ }^{\perp} \Sigma_{a b} .
$$

Thus, equation A13 is now

$$
\begin{aligned}
Z_{c b}= & \left(1+v_{s}\right) u_{b} u^{a} \nabla_{a} \mu_{c}-\epsilon v_{s} \nabla_{c} \mu_{b}-(\rho+p) u_{c}\left[u_{b} n^{a} \nabla_{a} \| \mathcal{A}+n_{b} u^{a} \nabla_{a}{ }^{\|} \mathcal{A}\right] \\
& +(\rho+p)\left[u_{b} n_{c} n^{a} \nabla_{a}\left(\frac{1}{3}{ }^{\|} \Theta+{ }^{\|} \Sigma\right)+u_{b} N_{c}^{a} \nabla_{a}\left(\frac{1}{3}{ }^{\|} \Theta-\frac{1}{2}{ }^{\|} \Sigma\right)+u_{b} \epsilon_{c}{ }^{a} \nabla_{a}{ }^{\|} \Omega+n_{c} n_{b} u^{a} \nabla_{a}\left(\frac{1}{3}{ }^{\|} \Theta+{ }^{\|} \Sigma\right)\right. \\
& \left.+N_{c b} u^{a} \nabla_{a}\left(\frac{1}{3}{ }^{\|} \Theta-\frac{1}{2}{ }^{\|} \Sigma\right)+\epsilon_{c b} u^{a} \nabla_{a}{ }^{\|} \Omega\right]+\grave{Z}_{c b}=0,
\end{aligned}
$$

with

$$
\begin{aligned}
& \grave{Z}_{c b} \equiv W_{c b}+(\rho+p)\left(\left[u_{b} \nabla_{a}{ }^{\perp} U_{c}{ }^{a}+u^{a} \nabla_{a}{ }^{\perp} U_{c b}\right]-{ }^{\|} \mathcal{A}\left[u_{b} \nabla_{a}\left(u_{c} n^{a}\right)+u^{a} \nabla_{a}\left(u_{c} n_{b}\right)\right]+\frac{1}{3} u_{b} \nabla_{a}\left(n^{a} n_{c}+N_{c}^{a}\right){ }^{\|} \Theta\right. \\
& \left.+{ }^{\|} \Sigma u^{a} \nabla_{a}\left(n_{b} n_{c}-\frac{1}{2} N_{b c}\right)+\frac{1}{3} u^{a} \nabla_{a}\left(n_{c} n_{b}+N_{b c}\right){ }^{\|} \Theta+{ }^{\|} \Sigma u_{b} \nabla_{a}\left(n^{a} n_{c}-\frac{1}{2} N_{c}^{a}\right)+{ }^{\|} \Omega\left[u_{b} \nabla_{a} \epsilon_{c}{ }^{a}+u^{a} \nabla_{a} \epsilon_{c b}\right]\right) .
\end{aligned}
$$

Explicitly $W_{c b}$ is given by

$$
\begin{aligned}
& W^{c b}=-R^{c}{ }_{a} u^{a} u^{b}(p+\rho)+R_{a}^{b}{ }_{a}^{c} u^{a} u^{d}(p+\rho)+u^{b}\left(1+v_{s}\right) \mu^{c} D_{a} u^{a}+u^{a} \mu^{c} D_{a} u^{b}+u^{a} v_{s} \mu^{c} D_{a} u^{b}-J^{a} u^{b} D^{c} E_{a}+J^{a} u_{a} D^{c} E^{b} \\
& +E^{b} u^{a} D^{c} J_{a}-E^{a} u^{b} D^{c} J_{a}+E^{b} J^{a} D^{c} u_{a}+u^{b} \mu^{a} D^{c} u_{a}+u^{b} v_{s} \mu^{a} D^{c} u_{a}+p D^{a} u^{b} D^{c} u_{a}+\rho D^{a} u^{b} D^{c} u_{a}-E^{a} J_{a} D^{c} u^{b} \\
& +u^{a} \mu_{a} D^{c} u^{b}+u^{a} v_{s} \mu_{a} D^{c} u^{b}+p D_{a} u^{a} D^{c} u^{b}+\rho D_{a} u^{a} D^{c} u^{b}-\epsilon_{a e}^{b}\left(J^{a} u^{e} D^{c} B_{d}+B^{a}\left(-u^{e} D^{c} J_{d}+J^{e} D^{c} u_{d}\right)\right) \\
& +u^{a} u^{b} \mu_{a} D^{c} v_{s}+\mu^{b} D^{c} v_{s} .
\end{aligned}
$$

Taking into account the above expressions, we obtain from the evolution equations the following projections:

$\mathbf{u}^{\mathrm{b}} \mathbf{u}^{\mathrm{c}} \mathbf{Z}_{\mathbf{c b}}$ :

$$
u^{b} u^{c} Z_{c b}=-u^{c} u^{a} \nabla_{a} \mu_{c}-(\rho+p) n^{a} \nabla_{a} \| \mathcal{A}+u^{b} u^{c} \grave{Z}_{c b}=0
$$

$\mathbf{n}^{\mathrm{b}} \mathbf{n}^{\mathrm{c}} \mathbf{Z}_{\mathbf{c b}}$

$$
n^{b} n^{c} Z_{c b}=v_{s} n^{c} n^{b} \nabla_{b} \mu_{c}+(\rho+p) u^{a} \nabla_{a}\left(\frac{1}{3} \| \Theta+{ }^{\|} \Sigma\right)+n^{b} n^{c} \grave{Z}_{c b}=0
$$

$\mathbf{n}^{\mathrm{c}} \mathbf{u}^{\mathrm{b}} \mathbf{Z}_{\mathbf{c b}}$ :

$$
n^{c} u^{b} Z_{c b}=-n^{c} u^{a} \nabla_{a} \mu_{c}-(\rho+p) n^{a} \nabla_{a}\left(\frac{1}{3}^{\|} \Theta+{ }^{\|} \Sigma\right)+n^{c} u^{b} \grave{Z}_{c b}=0 .
$$

$\mathbf{u}^{\mathrm{c}} \mathbf{n}^{\mathrm{b}} \mathbf{Z}_{\mathbf{c b}}$ :

$$
u^{c} n^{b} Z_{c b}=v_{s} u^{c} n^{b} \nabla_{b} \mu_{c}+(\rho+p) u^{a} \nabla_{a} \| \mathcal{A}+u^{c} n^{b} \grave{Z}_{c b}=0 .
$$

$\mathrm{g}^{\mathrm{cb}} \mathrm{Z}_{\mathrm{cb}}$ :

$$
g^{c b} Z_{c b}=\left(1+v_{s}\right) u^{b} u^{a} \nabla_{a} \mu_{b}+(\rho+p) n^{a} \nabla_{a}{ }^{\|} \mathcal{A}+v_{s} \nabla^{c} \mu_{c}+(\rho+p) u^{a} \nabla_{a}{ }^{\|} \Theta+g^{c b} \grave{Z}_{c b}=0 .
$$

$\mathbf{h}^{\mathrm{cb}} \mathrm{Z}_{\mathrm{cb}}$ :

$$
h^{c b} Z_{c b}=+v_{s} h^{c b} \nabla_{c} \mu_{b}+(\rho+p) u^{a} \nabla_{a}^{\|} \Theta+h^{c b} \grave{Z}_{c b}=0 .
$$

$\mathrm{N}^{\mathrm{cb}} \mathrm{Z}_{\mathrm{cb}}$

$$
N^{c b} Z_{c b}=+v_{s} N^{c b} \nabla_{c} \mu_{b}+2(\rho+p) u^{a} \nabla_{a}\left(\frac{1}{3} \| \Theta-\frac{1}{2}^{\|} \Sigma\right)+N^{c b} \grave{Z}_{c b}=0 .
$$


$\epsilon^{\mathbf{c b}} \mathbf{Z}_{\mathbf{c b}}$

$$
\epsilon^{c b} Z_{c b}=+2(\rho+p) u^{a} \nabla_{a} \| \Omega+\epsilon^{c b} \grave{Z}_{c b}=0 .
$$

The field $\mu_{b}$ can be decomposed as $\mu_{b}={ }^{\perp} \mu_{b}+\| \mu n_{b}$. This expression can be explicitly written as $\mu_{a}=\left(N_{a}^{b} \mu_{b}-u_{a} u^{b} \dot{\mu}_{b}\right)+$ $n_{a} n^{b} \mu_{b}$, thus the previous decomposition can be further refined. Using the torsion-free condition $\nabla_{a} \mu_{b}=\nabla_{b} \mu_{a}$ we obtain from $n^{c} u^{a}\left(\nabla_{a} \mu_{c}-\nabla_{c} \mu_{a}\right)=0$,

$$
u^{a} \nabla_{a}{ }^{\|} \mu={ }^{\perp} \mu_{c} u^{a} \nabla_{a} n^{c}+u^{a} n^{c} \nabla_{c}{ }^{\perp} \mu_{a}-{ }^{\|} \mu n_{a} n^{c} \nabla_{c} u^{a},
$$

which can be read as a propagation equation for " $\mu$.

Using equation (B9) we get from equation (B1), $\left(u^{b} u^{c} Z_{c b}=0\right)$ :

$$
(\rho+p) n^{a} \nabla_{a} \| \mathcal{A}=-u^{c} u^{a} \nabla_{a}{ }^{\perp} \mu_{c}+{ }^{\|} \mu^{\|} \mathcal{A}-u^{b} u^{c} \grave{Z}_{c b} .
$$

From equation $(\overline{\mathrm{B} 2}),\left(n^{b} n^{c} Z_{c b}=0\right)$ :

$$
(\rho+p) u^{a} \nabla_{a}\left(\frac{1}{3}{ }^{\|} \Theta+{ }^{\|} \Sigma\right)+v_{s} n^{b} \nabla_{b} \| \mu=v_{s}{ }^{\perp} \mu^{c} n^{b} \nabla_{b} n_{c}-n^{b} n^{c} \grave{Z}_{c b} .
$$

From equation $(\overline{\mathrm{B} 3}),\left(n^{c} u^{b} Z_{c b}=0\right)$ :

$$
u^{a} \nabla_{a} \| \mu+(\rho+p) n^{a} \nabla_{a}\left(\frac{1}{3}{ }^{\|} \Theta+{ }^{\|} \Sigma\right)={ }^{\perp} \mu_{c} u^{a} \nabla_{a} n^{c}+n^{c} u^{b} \grave{Z}_{c b} .
$$

Using equation (B9) in equation $(\mathrm{B} 3)$ one obtains

$$
(\rho+p) n^{a} \nabla_{a}\left(\frac{1}{3}{ }^{\|} \Theta+{ }^{\|} \Sigma\right)=-u^{a} n^{c} \nabla_{c}{ }^{\perp} \mu_{a}+{ }^{\|} \mu n_{a} n^{c} \nabla_{c} u^{a}+n^{c} u^{b} \grave{Z}_{c b} .
$$

From equation $\mathrm{B} 4\left(u^{c} n^{b} Z_{c b}=0\right)$ one gets:

$$
(\rho+p) u^{a} \nabla_{a}{ }^{\|} \mathcal{A}=-v_{s} u^{c} n^{b} \nabla_{b}{ }^{\perp} \mu_{c}-v_{s}{ }^{\|} \mu u^{c} n^{b} \nabla_{b} n_{c}-u^{c} n^{b} \grave{Z}_{c b},
$$

and using equation (B9) in equation (B4)

$$
(\rho+p) u^{a} \nabla_{a}{ }^{\|} \mathcal{A}+v_{s} u^{c} \nabla_{c}{ }^{\|} \mu=v_{s}{ }^{\perp} \mu_{b} u^{c} \nabla_{c} n^{b}-u^{c} n^{b} \grave{Z}_{c b} .
$$

From equation $(\mathrm{B} 5)\left(g^{c b} Z_{c b}=0\right)$ we get:

$$
\begin{aligned}
& (\rho+p) u^{a} \nabla_{a}{ }^{\|} \Theta+(\rho+p) n_{c} \nabla^{c \|} \mathcal{A}+v_{s} n_{c} \nabla^{c \|} \mu \\
& \quad=-\left(1+v_{s}\right) u^{b} u^{a} \nabla_{a}{ }^{\perp} \mu_{b}-v_{s} \nabla_{c}{ }^{\perp} \mu^{c}-\mu_{b} u^{b} u^{a} \nabla_{a} v_{s}-\left(1+v_{s}\right)^{\|} \mu u^{b} u^{a} \nabla_{a} n_{b}-v_{s}{ }^{\|} \mu \nabla^{c} n_{c}-g^{c b} \grave{Z}_{c b} .
\end{aligned}
$$

From equation $\mathrm{B} 6),\left(h^{c b} Z_{c b}=0\right)$ :

$$
(\rho+p) u^{a} \nabla_{a}{ }^{\|} \Theta+v_{s} n^{c} \nabla_{c}{ }^{\|} \mu=-v_{s} h^{c b} \nabla_{c}{ }^{\perp} \mu_{b}-v_{s}{ }^{\|} \mu \nabla_{c} n^{c}-h^{c b} \grave{Z}_{c b},
$$

From equation (B7), $\left(N^{c b} Z_{c b}=0\right)$ :

$$
(\rho+p) u^{a} \nabla_{a}\left(\frac{2}{3}{ }^{\|} \Theta-{ }^{\|} \Sigma\right)=-v_{s} N^{c b} \nabla_{c}{ }^{\perp} \mu_{b}-v_{s} N^{c b \|} \mu \nabla_{c} n_{b}-N^{c b} \grave{Z}_{c b} .
$$

From equation (B11), $\left(n^{b} n^{c} Z_{c b}=0\right)$ :

$$
(\rho+p) u^{a} \nabla_{a}\left(\frac{1}{3}{ }^{\|} \Theta+{ }^{\|} \Sigma\right)+v_{s} n^{b} \nabla_{b}{ }^{\|} \mu=v_{s}{ }^{\perp} \mu^{c} n^{b} \nabla_{b} n_{c}-n^{b} n^{c} \grave{Z}_{c b} .
$$

From equation $\mathrm{B} 6\left(h^{c b} Z_{c b}=0\right)$ :

$$
(\rho+p) u^{a} \nabla_{a}{ }^{\|} \Theta+v_{s} n^{c} \nabla_{c}{ }^{\|} \mu=-v_{s} h^{c b} \nabla_{c}{ }^{\perp} \mu_{b}-v_{s}{ }^{\|} \mu \nabla_{c} n^{c}-h^{c b} \grave{Z}_{c b} .
$$

Finally, we consider the equation $\frac{3}{2} \frac{\rho+p}{v_{s}}\left(\mathrm{~B} 11-\frac{1}{3}\right.$ B20 $)$, which, explicitly, is given by

$\frac{3}{2} \frac{(\rho+p)^{2}}{v_{s}} u^{a} \nabla_{a}{ }^{\|} \Sigma+(\rho+p) n^{c} \nabla_{c} \| \mu=\frac{3}{2} \frac{(\rho+p)}{v_{s}}\left(v_{s}{ }^{\perp} \mu^{c} n^{b} \nabla_{b} n_{c}-n^{b} n^{c} \grave{Z}_{c b}+\frac{1}{3}\left(v_{s} h^{c b} \nabla_{c}{ }^{\perp} \mu_{b}+v_{s} \| \mu \nabla_{c} n^{c}+h^{c b} \grave{Z}_{c b}\right)\right)$, 
and the equation $\frac{\rho+p}{3 v_{s}}$ B20 or

$$
\frac{(\rho+p)^{2}}{3 v_{s}} u^{a} \nabla_{a}{ }^{\|} \Theta+\frac{\rho+p}{3} n^{c} \nabla_{c} \| \mu=-\frac{\rho+p}{3 v_{s}}\left(v_{s} h^{c b} \nabla_{c}{ }^{\perp} \mu_{b}+v_{s}{ }^{\|} \mu \nabla_{c} n^{c}+h^{c b} \grave{Z}_{c b}\right) .
$$

For a LSS the equations Equations (B14), B8, B12, (B22, B21 constitute a symmetric hyperbolic system for the unknowns $(\|\mathcal{A},\| \Omega,\|\mu,\| \Theta, \| \Sigma)$. In Section (V A) we discussed an alternative set of symmetric hyperbolic system where the evolution equation for the radial acceleration $\mathcal{A}$ is coupled with the evolution of equation for the variable $Q \equiv \frac{2}{3} \Theta+2 \Sigma$. A symmetric hyperbolic system has, therefore, been given for the variables $\mathbf{v} \equiv(\rho, E, B, \mathcal{E}, \mathcal{B}, Q, T, \xi, \Phi, \Omega, \mathcal{A})$. Equation $(60 \mathrm{c})$ for the variable $\mathcal{A}$ has been recovered from the equation (B15) $-v_{s}(\mathrm{~B} 14)$. In a LSS equation (B14) is explicitly given by

$\mathcal{A}\left(1+v_{s}\right)(E j-\mathcal{A} \Theta(p+\rho))+j \varrho_{C}+E \varrho_{C}\left(\frac{2}{3} \Theta-\Sigma\right)-2 B \xi \varrho_{C}+(p+\rho) u^{a} D_{a} \mathcal{A}+\mu u^{a} D_{a} v_{s}+v_{s} u^{a} D_{a} \mu-E u^{a} D_{a} \varrho_{C}=0$,

and

$$
\begin{aligned}
\left(\frac{4}{3} \Theta+\Sigma\right)\left[\mathcal{A}(p+\rho)+\mu\left(1+v_{s}\right)\right]-\varrho_{C}\left[j+E\left(\Sigma+\frac{1}{3} \Theta\right)\right]+E j(\Phi-\mathcal{A})+\frac{3}{2}(p+\rho) \Sigma \Phi \\
+\Omega[2(p+\rho) \xi-2 B j]-E n^{a} D_{a} j+(\rho+p)\left(\frac{1}{3} n^{a} D_{a} \Theta+n^{a} D_{a} \Sigma\right)+u^{a} D_{a} \mu=0 .
\end{aligned}
$$

It is possible to show, using again the evolution equations and the constraints that the scalars $n^{c} n^{b} \grave{Z}_{c b}, h^{c b} \grave{Z}_{c b}, u^{c} n^{b} \grave{Z}_{c b}$ and $n^{c} u^{b} \grave{Z}_{c b}$ do not contain derivatives of the variables. These terms are discussed with more detail in the following Section.

\section{Appendix C: Evolution equations and hyperbolicity considerations}

The system consisting of equations (34), (52), (42), (44), (43), (26), (41), (38), 45a), and (45b) as discussed in Section (V) and equations (B14), (B12), (B21) and B22 for the variables

$$
\mathbf{v}^{(b)} \equiv\left(\rho, s, n,{ }^{\|} \Omega, \xi, \Phi, " E,{ }^{\|} B,\|\mathcal{B},\| \mathcal{E},\|\mathcal{A},\| \mu,\|\Sigma,\| \Theta\right)
$$

can be written explicitly as

$$
\begin{aligned}
& \dot{\rho}=-(\rho+p) \Theta+{ }^{\|} E^{\|} j, \\
& \dot{s}=\frac{1}{n T}{ }^{\|} E^{\|} j, \\
& \dot{n}=-n^{\|} \Theta, \\
& \| \dot{\Omega}={ }^{\|} \mathcal{A} \xi-{ }^{\|}{ }^{\|} \Theta^{\|} \Omega+{ }^{\|} \Sigma^{\|} \Omega, \\
& \dot{\xi}=\frac{1}{2}{ }^{\|} \mathcal{B}-\left(\frac{1}{3} \Theta-\frac{1}{2} \Sigma\right) \xi+\left({ }^{\|} \mathcal{A}-\frac{1}{2} \Phi\right){ }^{\|} \Omega, \\
& \dot{\Phi}=\left(\frac{1}{3}{ }^{\|} \Theta+\frac{1}{2}{ }^{\|} \Sigma\right)\left(2^{\|} \mathcal{A}+\Phi\right)-2 \xi^{\|} \Omega, \\
& { }^{\|} \dot{E}=2 \xi^{\|} B-\left(\frac{2}{3} \Theta-\Sigma\right){ }^{\|} E-{ }^{\|} j, \\
& { }^{\|} \dot{B}=-2 \xi^{\|} E-\left(\frac{2}{3} \Theta-\Sigma\right){ }^{\|} B, \\
& { }^{\|} \dot{\mathcal{B}}=\left(\frac{3}{2} \Sigma-\Theta\right){ }^{\|} \mathcal{B}-3 \xi^{\|} \mathcal{E}-\xi\left({ }^{\|} E^{2}+{ }^{\|} B^{2}\right), \\
& { }^{\|} \dot{\mathcal{E}}=\left(\frac{3}{2} \Sigma-\Theta\right){ }^{\|} \mathcal{E}+3 \xi^{\|} \mathcal{B}-\frac{1}{2}(\rho+p) \Sigma+\left(\frac{1}{2}{ }^{\|} \Sigma-\frac{1}{3}{ }^{\|} \Theta\right)\left({ }^{\|} E^{2}+{ }^{\|} B^{2}\right)-\frac{2}{3}{ }^{\|} E^{\|} j, \\
& (\rho+p) u^{a} \nabla_{a} \| \mathcal{A}=-v_{s}{ }^{\|} \mu u^{c} n^{b} \nabla_{b} n_{c}-u^{c} n^{b} \grave{Z}_{c b}, \\
& u^{a} \nabla_{a}{ }^{\|} \mu+(\rho+p) n^{a} \nabla_{a}\left(\frac{1}{3}{ }^{\|} \Theta+{ }^{\|} \Sigma\right)=n^{c} u^{b} \grave{Z}_{c b}, \\
& { }_{3} \frac{(\rho+p)^{2}}{v_{s}} u^{a} \nabla_{a}{ }^{\|} \Sigma+(\rho+p) n^{c} \nabla_{c} \| \mu=\frac{3}{2} \frac{(\rho+p)}{v_{s}}\left(\frac{1}{3}\left(v_{s}{ }^{\|} \mu \nabla_{c} n^{c}+h^{c b} \grave{Z}_{c b}\right)-n^{b} n^{c} \grave{Z}_{c b}\right), \\
& \frac{(\rho+p)^{2}}{3 v_{s}} u^{a} \nabla_{a}{ }^{\|} \Theta+\frac{\rho+p}{3} n^{c} \nabla_{c}{ }^{\|} \mu=-\frac{\rho+p}{3 v_{s}}\left(v_{s}{ }^{\|} \mu \nabla_{c} n^{c}+h^{c b} \grave{Z}_{c b}\right) .
\end{aligned}
$$

In the above expressions notice that $\dot{u}^{a}={ }^{\|} \mathcal{A} n^{a}, \quad \dot{n}^{a}=" \mathcal{A} u^{a}$. 
Moreover, one has that

$$
\begin{aligned}
& n^{c} n^{b} \grave{Z}_{c b}=-(\rho+p)\left({ }^{\|} \mathcal{A}^{2}+2^{\|} \Omega^{\|} \xi\right)+(\rho+p)\left[\frac{1}{3}(3 p-\rho)-\frac{1}{2}\left(p-\rho-{ }^{\|} E^{2}-{ }^{\|} B^{2}\right)-{ }^{\|} \mathcal{E}\right]+{ }^{\|} \mu^{\|} \mathcal{A}\left(1+v_{s}\right) \\
& +(\rho+p)\left(\frac{1}{3} \Theta+\Sigma\right)\left(\frac{4}{3} \| \Theta+{ }^{\|} \Sigma\right)-\epsilon^{\|} \mu \hat{v}_{s}+{ }^{\|} \hat{E} \varrho_{C}+{ }^{\|} E\left(\hat{\rho}_{C}+{ }^{\|} j\left(\frac{1}{3}{ }^{\|} \Theta+{ }^{\|} \Sigma\right)\right), \\
& h^{c b} \grave{Z}_{c b}=-{ }^{\|} \mathcal{A}(\rho+p)+\frac{1}{2}(\rho+p)\left(\rho+3 p+{ }^{\|} E^{2}+{ }^{\|} B^{2}\right)+{ }^{\|} \mu^{\|} \mathcal{A}\left(1+v_{s}\right)+(\rho+p)\left(\Theta^{2}+\left(\frac{1}{3} \Theta+\Sigma\right)^{2}+2\left(\frac{1}{3} \Theta-\frac{1}{2} \Sigma\right)^{2}\right) \\
& -\epsilon^{\|} \mu \hat{v}_{s}+{ }^{\|} \hat{E} \varrho_{C}+{ }^{\|} E\left(\hat{\rho}_{C}+\varrho_{C} \Phi+{ }^{\|} j \Theta\right), \\
& u^{c} n^{b} \grave{Z}_{c b}=-{ }^{\|} \mathcal{A}(\rho+p)\left({ }^{\|} \Sigma+\frac{1}{3} \Theta\right)+{ }^{\|} \mathcal{A}(\rho+p)\left(\frac{4}{3} \Theta+\Sigma\right)-\epsilon^{\|} \mu \dot{v}_{s}+{ }^{\|} \dot{E}_{\varrho_{C}}+{ }^{\|} E \dot{\rho}_{C}+{ }^{\|} E^{\|} \mathcal{A}^{\|} j, \\
& n^{c} u^{b} \grave{Z}_{c b}=-(\rho+p)\left(\frac{1}{2} \Phi \Sigma+{ }^{\|} \mathcal{A}\left(\frac{1}{3} \Theta+\Sigma\right)+2^{\|} \Omega^{\|} \xi\right)-" \mu\left(1+v_{s}\right)\left(\frac{4}{3} \Theta+\Sigma\right)+\dot{v}_{s} \| \mu-{ }^{\|} \hat{E}^{\|} j-{ }^{\|} E\left(\| \hat{j}+\varrho_{C}\left(\| \Sigma+\frac{1}{3} \Theta\right)\right) .
\end{aligned}
$$

To write down the above explicit expressions for $\left(n^{c} n^{b} \grave{Z}_{c b}\right),\left(n^{c} u^{b} \grave{Z}_{c b}\right)$ and $\left(h^{c b} \grave{Z}_{c b}\right),\left(u^{c} n^{b} \grave{Z}_{c b}\right)$ we have used, again, the evolution equation (C7) and the constraint equations (27) for "E. With regards to the term $\hat{v}_{s}$ in $\left(n^{c} n^{b} \grave{Z}_{c b}\right)$ and $\left(h^{c b} \grave{Z}_{c b}\right)$ and $\dot{v}_{s}$ in $\left(u^{c} n^{b} \grave{Z}_{c b}\right)$ and $\left(n^{c} u^{b} \grave{Z}_{c b}\right)$, involving the derivatives of $v_{s}$ we note the following: the definition $v_{s}=d p / d \rho$ has been used by setting an appropriate equation of state $p=p(\rho)$. For example, in te case of a polytropic equation of state one has that $v_{s}=$ and therefore set aside the terms $\hat{v}_{s}$ and $\dot{v}_{s}$. Otherwise, one can consider a generic equation of state $\rho=\rho(p)$ such that $d v_{s} / d \rho=d^{2} p / d \rho^{2} \neq 0$ and $\nabla_{a} v_{s}=\left(d^{2} p / d \rho^{2}\right) \nabla_{a} \rho$ and assume $\left(d^{2} p / d \rho^{2}\right)=$ constant. In the more general case we refer to the discussion in[11] - see Section 14.4. In fact, as discussed in Section IV] for the more general case of a fluid which is not isentropic, i. e. $s \neq$ constant, the equation of state should be written in the form $p=p(\rho, s)$ - see [10] and references therein. This means that when considering derivatives of the pressure $p$, it should have been taken into account that $\nabla_{a} p=v_{s} \nabla_{a} \rho+(\partial p / \partial s) \nabla_{a} s$, where the time evolution of the entropy is governed by equation $\mathrm{C} 2$.

The evolution equations under consideration contains terms involving $\nabla_{a} v_{s}$ and $\nabla_{a} s$. The evolution equation for the new variable $s_{a} \equiv \nabla_{a} s$ can be obtained by covariant differentiating equation (C2), commuting covariant derivatives and using, again, the evolution equations - see [10].

Concerning the terms $\dot{\rho}_{C}$ in $\left(u^{c} n^{b} \grave{Z}_{c b}\right)$ and $\hat{\rho}_{C}$ in $\left(n^{c} n^{b} \grave{Z}_{c b}\right)$ and $\left(h^{c b} \grave{Z}_{c b}\right)$, one can assume, for example, that the charge density $\varrho_{C}$ is a function of the matter density $\varrho_{C}=\varrho_{C}(\rho)$. Here we assume it to be a constant multiple of the fluid density, $\rho=\varrho \varrho_{C}$. In this case one can use again equations (C1) and (??) and the definition of $v_{s}$. Note that the density $\varrho_{C}$ appears in the constraint equations for the electric field and the pressure. The discussion of Sections (V) and $\mathrm{VA}$ shows that $\varrho_{C}$ is only involved in the evolution equation for the radial acceleration. Here $\varrho_{C}$ is inherited from the term " $\mu$ containing information from the propagation of the matter density and pressure. Finally, we can use equation (27) and (56) for " $\hat{j}$ in $\left(n^{c} u^{b} \grave{Z}_{c b}\right)$, assuming $" ~ j=" ~ j(" E)$. In particular, we take " $j=\| E \sigma_{J}$.

This system (C1)-C13) can be written matricially as $\mathbf{A}_{(a)(b)}^{a} \partial_{a} \mathbf{v}^{(b)}=\mathbf{B}_{(a)(b)} \mathbf{v}^{(b)}$. It is convenient to write $\mathbf{v}^{(b)}=$ $\left(\mathbf{v}^{(i)}, \mathbf{v}^{(A)}\right)$ where

$$
\mathbf{v}^{(i)}=\left({ }^{\|} E,{ }^{\|} B,{ }^{\|} \mathcal{B},{ }^{\|} \mathcal{E}, s, n, \rho,{ }^{\|} \Omega, \xi, \Phi,{ }^{\|} \mathcal{A}\right), \quad \mathbf{v}^{(A)}=\left({ }^{\|} \mu,{ }^{\|} \Theta,{ }^{\|} \Sigma\right) .
$$

Thus it follows that

$$
\begin{aligned}
& A_{(i)(i)}^{a}=u^{a}, \quad A_{(i)(A)}^{a}=A_{(A)(i)}^{a}=0, \\
& A_{(i)(j)}^{a}=A_{(j)(i)}^{a}=0 \quad i \neq j,
\end{aligned}
$$

while for $\left(" \mu,{ }^{\|} \Theta,{ }^{\|} \Sigma\right)$ :

$$
\begin{aligned}
& A_{(\mu)(\mu)}^{a}=u^{a}, \quad A_{(\Theta)(\Theta)}^{a}=\frac{(\rho+p)^{2}}{3 v_{s}} u^{a}, A_{(\Sigma)(\Sigma)}^{a}=\frac{3(\rho+p)^{2}}{2 v_{s}} u^{a}, \\
& A_{(\mu)(\Theta)}^{a}=\frac{(\rho+p) n^{a}}{3}, \quad A_{(\Theta)(\mu)}^{a}=\frac{(\rho+p) n^{a}}{3}, \quad A_{(\Sigma)(\mu)}^{a}=(\rho+p) n^{a}, \\
& A_{(\mu)(\Sigma)}^{a}=(\rho+p) n^{a}, \quad A_{(\Theta)(\Sigma)}^{a}=0, \quad A_{(\Sigma)(\Theta)}^{a}=0 .
\end{aligned}
$$




\section{Appendix D: Details on the subclasses of the solutions}

Subclasses of the $\mathfrak{I}$-class $(\mathcal{A}, \mathbf{T})$ In what follows we analyse in further detail the following subcases of the $\operatorname{configuration}(\mathcal{A} \mathbf{T})$ :

$(\mathcal{A}$ T Q $): \quad$ in this case the configuration is defined by the conditions $\mathcal{A}=0, \Sigma=0$ and $\Theta=0$. The matter density evolution only depends on the electric field and the current density. The evolution of the electromagnetic fields is regulated by the twist $\xi$ of the 2-sheet. Moreover, the radial vorticity is constant during the motion -i.e. $\dot{\Omega}=0$. From the evolution equations for $T$ and $Q$ we obtain, respectively, the conditions $\mathcal{C}(\mathcal{A}, T)$ and $\mathcal{C}(\mathcal{A}, Q)$. From these relations we find $\mathcal{E}=p+\frac{1}{3} \rho-2 \Omega^{2}$ and $4 \Omega^{2}=B^{2}+E^{2}+3 p+\rho$. This case has at least one zero eigenvalue associated with the vorticity. This eigenvalue has multiplicity 2 . The sum of the eigenvalues of the associated 7-rank matrix is $\operatorname{Tr} \stackrel{\circ}{\mathbf{B}}=-\sigma_{J}<0[51$ ]

$(\mathcal{A} \mathbf{T} \boldsymbol{\Phi})$ : in this case one has $\dot{\Omega}=0$, and $\mathcal{C}(\mathcal{A}, T)$. The conditions $\mathcal{A}=0$ and $\Phi=0$ imply from equations (59f), (59g) ) either (i) $\Omega=0$ or (ii) $\xi=0$ and $\mathcal{B}=0$. We consider these two subcases separately:

(i) For $(\mathcal{A} \mathbf{T} \boldsymbol{\Phi} \Omega)$ the system is characterised by the condition $\mathcal{C}(\mathcal{A}, T, \Omega)$. From the criterion on the trace one can deduce that the system is unstable if $\stackrel{\Theta}{\Theta} \leq-\left(\sigma_{J}\right) /\left(3+v_{s}\right)$. Now, considering the determinant of the reduced $7 \times 7$-linearised matrix [52] the necessary condition for stability is that $\operatorname{det} \stackrel{\circ}{\mathbf{B}}<$. Combining this condition with the criterion of the trace and using the fact that $\stackrel{\circ}{p}=\stackrel{\circ}{\rho} v_{s}$ we obtain for stable configuration the following conditions:

(ia) for $\stackrel{\circ}{\rho}<\stackrel{\circ}{\rho}_{s}$ then one has that $\stackrel{\circ}{\Sigma} \in\left(-\stackrel{\circ}{\Sigma}_{s},+\stackrel{\circ}{\Sigma}_{s}\right)$ and $\mathfrak{Q}\left(\stackrel{\circ}{\mathcal{B}}, \stackrel{\circ}{\xi}, \stackrel{\circ}{E}^{2}\right)<0$, or $\stackrel{\circ}{\Sigma} \in\left(-\stackrel{\circ}{\Sigma}_{x},-\stackrel{\circ}{\Sigma}_{s}\right) \cup\left(\stackrel{\circ}{\Sigma}_{s}, \infty\right)$ and $\mathfrak{Q}\left(\dot{\mathcal{B}}, \stackrel{\circ}{\xi}, \stackrel{\circ}{E}^{2}\right)>0$;

(ib) For $\stackrel{\circ}{\rho} \geq \rho_{s}$ then one has $\stackrel{\circ}{\Sigma} \in\left(-\stackrel{\circ}{\Sigma}_{x},+\stackrel{\circ}{\Sigma}_{s}\right)$ and $\mathfrak{Q}\left(\stackrel{\circ}{\mathcal{B}}, \stackrel{\circ}{\xi}, \stackrel{\circ}{E}^{2}\right)<0$, or $\stackrel{\circ}{\Sigma} \in\left(+\stackrel{\circ}{\Sigma}_{s}, \infty\right)$ and $\mathfrak{Q}\left(\stackrel{\circ}{\mathcal{B}}, \stackrel{\circ}{\xi}, \stackrel{\circ}{E}^{2}\right)>$ 0 . With $\stackrel{\circ}{\xi} \neq 0, \mathfrak{Q}\left(\mathcal{B}, \xi, E^{2}\right) \equiv 3 \mathcal{B} \xi+2 E^{2} \sigma_{J}$ and considering the $(\mathbf{L E V})_{\boldsymbol{\Sigma}}$

We note that the reference matter density and shear $\rho_{s}$ and $\Sigma_{x}$, depend on the constants $\left(v_{s}, \sigma_{J}\right)$ and the limit $\Sigma_{s}$ depends only on the matter density and the square of the sound velocity.

(ii) $(\mathcal{A} \mathbf{T} \mathbf{\Phi}, \xi, \mathcal{B})$ : in this case the Maxwell equations simply became $\dot{B}=0$ and $\dot{E}=-j$. Moreover $\dot{\Omega}=0$ and the condition $\mathcal{C}(\mathcal{A}, T)$ holds. The corresponding reduced system has six unknowns. We notice here the eigenvalues: $\lambda_{0}=0$ with a subspace of dimension 3 and

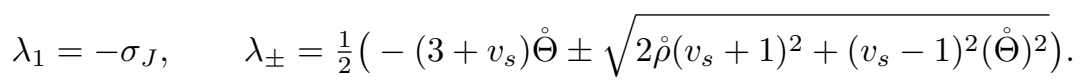

The conditions on the sign of $\lambda_{ \pm}$imposes severe restrictions on the radial expansion. These depend on the sound velocity and the background density matter: the condition $\lambda_{ \pm}<0$ (for the system stability) is satisfied if $\stackrel{\circ}{\Theta}>\sqrt{\left(1+v_{s}\right) \stackrel{\rho}{\rho} / 2}$.

$(\mathcal{A} \mathbf{T} \xi)$ : this case implies the equations $\dot{B}=\dot{\mathcal{B}}=\dot{\Omega}=\dot{\Phi}=0$ while the electric field satisfies $\dot{E}=-j$. Moreover, one has the condition $\mathcal{C}(\mathcal{A}, \xi)$ with $\mathcal{C}(\mathcal{A}, T)$. We study the associated $8 \times 8$ matrix: the eigenvalues of this matrix are $\lambda_{ \pm}$and $\lambda_{i}$ as defined for the case $(\mathcal{A} \mathbf{T} \boldsymbol{\Phi}, \xi, \mathcal{B})$ and a zero eigenvalue with multiplicity 5 due to the variables $(B, \mathcal{B}, \Omega, \Phi)$.

$(\mathcal{A} \mathbf{T} \Omega)$ : in this case one has $\dot{\Phi}=0$. From equation 60 a one finds $\mathcal{C}(\mathcal{A}, T, \Omega)$. The time evolution of the electric and magnetic fields are regulated by the twisting $\xi$ of the 2 -sheet - see equations $(59 \mathrm{~b})-(59 \mathrm{c})$ ). The temporal evolution of $\xi$ is fixed explicitly by the magnetic part of the Weyl tensor - see equation (59g). On the other hand, from the trace of the associated rank 7 matrix we infer that the system is unstable if $\stackrel{\circ}{\Sigma}<-\Sigma_{x}$. In order to obtain necessary conditions for stability one requires $c_{7}=-\operatorname{Tr} \stackrel{\circ}{\mathbf{B}} 0$, and $c_{1}>0$. These inequalities lead to the following cases with $(\mathbf{L E V})_{\Theta}$ :

(i) if $\stackrel{\circ}{\rho}<\stackrel{\circ}{\rho}_{s}$ then $\stackrel{\circ}{\Theta} \in\left(-\stackrel{\circ}{\Theta}_{x},-\stackrel{\circ}{\Theta}_{s}\right) \cup\left(+\stackrel{\circ}{\Theta}_{s}, \infty\right)$ and $\stackrel{\circ}{\xi} \neq 0$, or $\stackrel{\circ}{\Theta} \in\left(-\stackrel{\circ}{\Theta}_{x},+\stackrel{\circ}{\Theta}_{s}\right)$ and $\stackrel{\circ}{\xi} \mathcal{B}<0$;

(ii) if $\stackrel{\circ}{\rho} \geq \rho_{s}$ then $\stackrel{\circ}{\Theta} \in\left(-\stackrel{\circ}{\Theta}_{s},+\stackrel{\circ}{\Theta}_{s}\right)$ and $\stackrel{\circ}{\xi} \dot{\mathcal{B}}<0$, or $\stackrel{\circ}{\Theta} \in\left(+\stackrel{\circ}{\Theta}_{s}, \infty\right)$ and $\stackrel{\circ}{\xi} \neq 0$

Subclasses of the $\mathfrak{I} \mathfrak{I}$-class $(\mathcal{A}, \mathbf{Q})$ In this subsection we focus on the configurations with $\frac{1}{3} \Theta=-\Sigma$. Taking into account the results on the system $(\mathcal{A} \mathbf{Q})$, we consider the following subcases.

$(\mathcal{A} \mathbf{Q} \Phi)$ : the condition $\mathcal{C}(\mathcal{A}, Q)$ holds. Moreover, the conditions $\mathcal{A}=0$ and $\Phi=0$, imply from equation $(59 \mathrm{f})$ two subcases, (i) $\Omega=0$ and (ii) $(\xi=0, \mathcal{B}=0)$, respectively.

(i) $(\mathcal{A} \mathbf{Q} \boldsymbol{\Phi} \mathbf{\Omega})$ : from the trace of the reduced rank 7 matrix the configuration is bound to be unstable if $\stackrel{\circ}{\Sigma}^{\circ} \Sigma_{s} \equiv 2 \sigma_{J} / 3\left(15+2 v_{s}\right)$. On the other hand, studying the sign of the characteristic polynomial 
coefficient $c_{5}<0$ we infer that to obtain stability: for $\stackrel{\circ}{\mathcal{E}}<\dot{\mathcal{E}}_{p}$ and (a) $\stackrel{\circ}{T}>-2 \sigma_{J} /\left(15+2 v_{s}\right)$ with $\stackrel{\circ}{\xi}<-\stackrel{\circ}{\xi}_{p}$ or $\stackrel{\circ}{\xi}>\stackrel{\circ}{\xi}_{p}$, or (b) $\stackrel{\circ}{\mathcal{E}}<\stackrel{\circ}{\mathcal{E}}_{p}$ and $\stackrel{\circ}{T}>\stackrel{\circ}{T}_{p}$. Otherwise, for $\stackrel{\circ}{\mathcal{E}} \geq \stackrel{\circ}{\mathcal{E}}_{p}$ with $\stackrel{\circ}{T}>-2 \sigma_{J} /\left(15+2 v_{s}\right)$, with the following definitions:

$$
\begin{aligned}
& \stackrel{\circ}{\mathcal{E}}_{p} \equiv\left(\frac{5}{6}+\frac{5}{2} v_{s}+v_{s}^{2}\right) \stackrel{\rho}{\rho}+\frac{2\left(50+v_{s}\left(15+2 v_{s}\right)\right) \sigma_{J}^{2}}{\left(15+2 v_{s}\right)^{2}}, \\
& \stackrel{\circ}{T}_{p} \equiv \frac{-3\left(13+2 v_{s}\right) \sigma_{J}+\sqrt{6\left(95+26 v_{s}\right)\left(\left(5+3 v_{s}\left(5+2 v_{s}\right)\right) \stackrel{\rho}{\rho}-6 \dot{\mathcal{E}}\right)+9\left(13+2 v_{s}\right)^{2} \sigma_{J}^{2}}}{285+78 v_{s}}, \\
& \stackrel{\circ}{\xi}_{p}=\frac{\sqrt{39} \sqrt{2\left(5+3 v_{s}\left(5+2 v_{s}\right)\right) \stackrel{\circ}{\rho}-12 \dot{\mathcal{E}}-3 \dot{T}^{2}\left(95+26 v_{s}\right)-6 \stackrel{\circ}{ }\left(13+2 v_{s}\right) \sigma_{J}}}{78},
\end{aligned}
$$

with $T=-3 \Sigma$. Once again, the limiting conditions on the radial part of the shear of the 3 -sheet, $\Sigma$, and the electric part of the Weyl tensor is completely regulated by the reference density and the constants $\left(\sigma_{J}, v_{s}\right)$. This stability conditions can be alternatively expressed as follows:

$\rho>0, \quad \stackrel{\circ}{\Sigma}>\Sigma_{s}, \quad$ and $\quad \stackrel{\circ}{\xi}^{2}>\stackrel{\circ}{\xi}_{s}^{2} \equiv \frac{1}{52}\left(2\left(\stackrel{\circ}{B}^{2}+\stackrel{\circ}{E}^{2}\right)+4\left(1+v_{s}\left(3+v_{s}\right)\right) \stackrel{\circ}{\rho}+3 \stackrel{\circ}{\Sigma}\left(2 \sigma_{J}\left(13+2 v_{s}\right)-3\left(95+26 v_{s}\right) \stackrel{\circ}{\Sigma}\right)\right)$.

(ii) $(\mathcal{A} \mathbf{Q} \Phi \xi \mathcal{B})$ : with the condition $\mathcal{C}(\mathcal{A}, Q)$, while the time evolution of the radial projected vorticity is entirely regulated by the radial part of the shear - see equation $(59 \mathrm{~h})$. The system, with a rank 6 matrix, is unstable if $\stackrel{\circ}{\Sigma} \geq \Sigma_{s} \equiv 2 \sigma_{J} / 3\left(13+2 v_{s}\right)$. Otherwise, it is stable if (a) $\dot{\mathcal{E}}<\dot{\mathcal{E}}_{p}$ with $\stackrel{\circ}{T}>-\left(2 \sigma_{J}\right) /\left(13+2 v_{s}\right)$ and $\Omega>\Omega_{p} \cup \stackrel{\Omega}{\Omega}<-\AA_{p}$ or $-\AA_{\Omega} \leq \AA \leq \AA_{p}$ with $\stackrel{\circ}{T}>\AA_{p}$; (b) $\mathcal{E} \geq \dot{\mathcal{E}}_{p}$ and $\stackrel{\circ}{T}>-\left(2 \sigma_{J}\right) /\left(13+2 v_{s}\right)$, where

$$
\begin{aligned}
& \stackrel{\circ}{\Omega}_{p} \equiv \frac{1}{2 \sqrt{6}} \sqrt{-3 \mathcal{E}+2\left(2+3 v_{s}\left(2+v_{s}\right)\right) \stackrel{\circ}{\rho}+\frac{6\left(73+26 v_{s}+4 v_{s}^{2}\right) \sigma_{J}^{2}}{\left(13+2 v_{s}\right)^{2}}} \\
& \stackrel{\circ}{T}_{p} \equiv \frac{-3\left(11+2 v_{s}\right) \sigma_{J}}{210+66 v_{s}}+ \\
& +\frac{\sqrt{3} \sqrt{8\left(35+11 v_{s}\right)\left(2+3 v_{s}\left(2+v_{s}\right)\right) \stackrel{\rho}{ }+3\left(11+2 v_{s}\right)^{2} \sigma_{J}^{2}-12 \mathcal{E}\left(35+11 v_{s}\right)-96\left(35+11 v_{s}\right) \AA^{2}}}{210+66 v_{s}} \\
& \stackrel{\circ}{\mathcal{E}}_{p} \equiv \frac{2}{3}\left(2+3 v_{s}\left(2+v_{s}\right)\right) \stackrel{\rho}{\rho}+\frac{2\left(73+26 v_{s}+4 v_{s}^{2}\right) \sigma_{J}^{2}}{\left(13+2 v_{s}\right)^{2}} .
\end{aligned}
$$

Alternatively, these conditions can be reexpressed as

$$
\stackrel{\circ}{\Sigma}<\Sigma_{s} \quad \text { and } \quad \stackrel{\circ}{\Omega}^{2}>\left(\stackrel{\circ}{B}^{2}\left(3 \stackrel{\circ}{E}^{2}-2\right)-2 \stackrel{\circ}{E}^{2}+\left(3+v_{s}\left(9+4 v_{s}\right)\right) \stackrel{\circ}{\rho}+6 \stackrel{\circ}{\Sigma}\left(\left(11+2 v_{s}\right) \sigma_{J}-3\left(35+11 v_{s}\right) \stackrel{\circ}{\Sigma}\right)\right) / 16 .
$$

$(\mathcal{A} \mathbf{Q} \xi)$ : in this case one has $\Sigma=-\Theta / 3$ with $\mathcal{C}(\mathcal{A}, \xi)$ from equation $59 \mathrm{~g}$ ) and $\mathcal{C}(\mathcal{A} Q)$. The radial shear is the only kinematical variable that explicitly regulates the time evolution of the variables $(E, B, \rho, \Phi, \mathcal{E}, \mathcal{B})$. The radial vorticity and the shear are related by the two evolution equations (59h) and (60a)), respectively. This is a rank 8 matrix problem. The system is unstable if $\Sigma^{\circ} \geq\left(2 \sigma_{J}\right) /\left(51+6 v_{s}\right)$. The system is stable if either (a) $\dot{\mathcal{E}}<\dot{\mathcal{E}}_{p}$ and $\stackrel{\circ}{T}>\stackrel{\circ}{T}_{p}$ or $-2 \sigma_{J} /\left(17+2 v_{s}\right)<\stackrel{\circ}{T}<\stackrel{\circ}{T}_{p}$ and $\stackrel{\Omega}{\Omega}<-\AA_{p} \cup \stackrel{\circ}{\Omega}>\AA_{p}$; or (b) $\mathcal{E} \geq \stackrel{\circ}{\mathcal{E}}_{p}$ and $\stackrel{\circ}{T}>-2 \sigma_{J} /\left(17+2 v_{s}\right)$. In the above conditions we used the following definitions:

$$
\begin{aligned}
& \stackrel{\Omega}{\Omega}_{p} \equiv \frac{\sqrt{3} \sqrt{4\left(2+3 v_{s}\left(2+v_{s}\right)\right) \stackrel{\circ}{\rho}-6 \stackrel{\mathcal{E}}{ }-15 \stackrel{\circ}{2}^{2}\left(25+6 v_{s}\right)-6 \stackrel{\circ}{T}\left(15+2 v_{s}\right) \sigma_{J}}}{12}, \\
& \stackrel{\circ}{\mathcal{E}}_{p} \equiv \frac{2}{3}\left(2+3 v_{s}\left(2+v_{s}\right)\right) \stackrel{\circ}{\rho}+\frac{4\left(65+v_{s}\left(17+2 v_{s}\right)\right) \sigma_{J}^{2}}{\left(17+2 v_{s}\right)^{2}}, \\
& \stackrel{\circ}{T}_{p} \equiv \frac{\sqrt{30\left(25+6 v_{s}\right)\left(2\left(2+3 v_{s}\left(2+v_{s}\right)\right) \stackrel{\circ}{\rho}-3\left(15+2 v_{s}\right) \sigma_{J}-3 \dot{\mathcal{E}}\right)+9\left(15+2 v_{s}\right)^{2} \sigma_{J}^{2}}}{375+90 v_{s}} .
\end{aligned}
$$


Alternatively, these conditions can be written as

$$
\stackrel{\circ}{\Sigma}<2 \sigma_{J} /\left(51+6 v_{s}\right), \quad \text { and } \quad \stackrel{\circ}{\Omega}^{2}>\frac{1}{16}\left(\stackrel{\circ}{B}^{2}+\stackrel{\circ}{E}^{2}+\left(3+v_{s}\left(9+4 v_{s}\right)\right) \stackrel{\circ}{\rho}+3 \stackrel{\circ}{\Sigma}\left(\left(30+4 v_{s}\right) \sigma_{J}-15\left(25+6 v_{s}\right) \stackrel{\circ}{\Sigma}\right)\right) .
$$

$(\mathcal{A} \mathbf{Q} \boldsymbol{\Omega})$ : in this case one has that $T=-3 \Sigma$ and $\Sigma=-\Theta / 3$ and the condition $\mathcal{C}(\mathcal{A}, Q): B^{2}+E^{2}+2 \mathcal{E}=$ $-(p+\rho / 3)$ holds. The system is unstable if $\Sigma^{\circ} \geq \Sigma_{s} \equiv \sigma_{J} / 3\left(8+v_{s}\right)$. A stable system meets the following conditions: (a) $\stackrel{\circ}{\mathcal{E}}<\stackrel{\circ}{\mathcal{E}}_{p}$, with $\stackrel{\circ}{T}>\stackrel{\circ}{T}_{p}$ or $-3 \Sigma_{s}<\stackrel{\circ}{T} \leq \stackrel{\circ}{T}_{p}$ and $\left(\stackrel{\circ}{\xi}<-\stackrel{\circ}{\xi}_{p}, \stackrel{\circ}{\xi}>\stackrel{\circ}{\xi}_{p}\right)$; or (b) $\dot{\mathcal{E}} \geq \stackrel{\circ}{\mathcal{E}}_{p}$ and $\stackrel{\circ}{T}>-3 \Sigma_{s}$. In the above expressions we have used the definitions

$$
\begin{aligned}
& \stackrel{\circ}{T}_{p} \equiv \frac{-3\left(7+v_{s}\right) \sigma_{J}+\sqrt{3\left(55+14 v_{s}\right)\left(\left(5+3 v_{s}\left(5+2 v_{s}\right)\right) \stackrel{\rho}{\rho}-6 \mathcal{E}\right)+9\left(7+v_{s}\right)^{2} \sigma_{J}^{2}}}{165+42 v_{s}}, \\
& \stackrel{\circ}{\xi}_{p} \equiv \frac{\sqrt{\left(5+3 v_{s}\left(5+2 v_{s}\right)\right) \stackrel{\circ}{\rho}-6 \stackrel{\circ}{\mathcal{E}}-3 \stackrel{\circ}{T}^{2}\left(55+14 v_{s}\right)-6 \stackrel{\circ}{T}\left(7+v_{s}\right) \sigma_{J}}}{\sqrt{78}}, \\
& \stackrel{\circ}{\mathcal{E}}_{p} \equiv \frac{\left(8+v_{s}\right)^{2}\left(5+3 v_{s}\left(5+2 v_{s}\right)\right) \stackrel{\circ}{\rho}+3\left(57+2 v_{s}\left(8+v_{s}\right)\right) \sigma_{J}^{2}}{6\left(8+v_{s}\right)^{2}} .
\end{aligned}
$$

The conditions can be expressed, alternatively, as

$$
\stackrel{\circ}{\Sigma}<\Sigma_{s} \quad \text { and } \quad 16 \dot{\circ}^{2}>\left(\stackrel{\circ}{B}^{2}+\stackrel{\circ}{E}^{2}+2\left(1+v_{s}\left(3+v_{s}\right)\right) \stackrel{\circ}{\rho}-9\left(55+14 v_{s}\right) \stackrel{\circ}{\Sigma}^{2}+6\left(7+v_{s}\right) \stackrel{\circ}{\Sigma} \sigma_{J}\right) .
$$

Subclasses of the $\mathfrak{I} \mathfrak{V}$-class $(\mathcal{A}, \xi)(\mathcal{A} \xi \Omega)$ : the assumptions $\mathcal{A}=0, \xi=0$ and $\Omega=0$, lead from equation $(59 \mathrm{~g})$ to the condition $\mathcal{C}(\mathcal{A} \xi, \Omega): \mathcal{B}=0$. Thus, we analyse the case: $(\mathcal{A} \xi \boldsymbol{\Omega} \mathcal{B})$, with a rank 7 matrix. The system is unstable if $\stackrel{\odot}{\Theta} \leq-3\left(2 \sigma_{J}+\left(v_{s}-5\right) \stackrel{\circ}{\Sigma}\right) / 4\left(7+v_{s}\right)$.

The case $\mathcal{A}=0, T=0$, and $Q=0(\mathcal{A} \mathbf{T} \mathbf{Q} \mathbf{\Phi})$ : in this special case we assume the radial acceleration to be zero. In addition, the expansion of the 2 -sheet $\Phi$ vanishes and also $\Sigma=\Theta=0$. It is worth noting that the assumption $\mathcal{A}=\Sigma=\Theta=\Phi=0$ implies from equation (59f) that $\xi \Omega=0$-that is, the twist of the 2-sheet $\xi$ together with the magnetic part of the Weyl tensor vanish. This system can only accelerate in the radial direction or otherwise radially projected vorticity will vanish. The basic assumptions in this case directly lead to $\dot{\Omega}=0$, $\dot{\xi}=\mathcal{B} / 2$ and the conditions $\mathcal{C}(\mathcal{A}, T)$ and $\mathcal{C}(\mathcal{A}, Q)$ hold. Thus, two subcases occur:

(i) $(\mathcal{A} \mathbf{T} \mathbf{Q} \mathbf{\Phi} \xi \mathcal{B})$; in this case one further has $\dot{B}=\dot{\Omega}=0, \dot{E}=-j$. The only non zero eigenvalue is $\lambda=-\sigma_{J}$.

(ii) $(\mathcal{A} \mathbf{T} \mathbf{Q} \mathbf{\Phi} \boldsymbol{\Omega})$; in this case, it can be shown that the trace of the reduced matrix $\operatorname{Tr} \mathbf{B}^{-}=-\sigma_{J}<0$ and this system has a zero eigenvalue. Nevertheless, in this case the conditions $\mathcal{C}(\mathcal{A}, T, \Omega)$ and $\mathcal{C}(\mathcal{A}, Q)$ is $B^{2}+E^{2}+2 \mathcal{E}=-\left(p+\frac{\rho}{3}\right)<0$ holds. These conditions are inconsistent with the type of equation of state considered in this work. We notice that this situation always occurs when the two conditions $\mathcal{C}(\mathcal{A}, T, \Omega)$ and $\mathcal{C}(\mathcal{A}, Q)$ must be satisfied simultaneously.

$(\mathcal{A} \mathbf{T} \mathbf{Q} \xi): \quad$ in this case the problem is simplifies considerably and we find the relations $\mathcal{C}(\mathcal{A}, \xi), \mathcal{C}(\mathcal{A}, T)$ and $\mathcal{C}(\mathcal{A}, Q)$. Moreover, one has that $\dot{E}=-j$. The evolution equations $\dot{B}=0, \dot{\mathcal{B}}=0, \dot{\Omega}=0$, and $\dot{\Phi}=0$ give rise to repeated zero eigenvalues. In addition one has the eigenvalue $\lambda=-\sigma_{J}<0$.

$(\mathcal{A}$ T Q $\Omega)$ : in this case one readily has that $\dot{\Phi}=0$. The associated rank 5 matrix has a zero eigenvalue with multiplicity 2. In addition, one has that $c_{6}=\operatorname{Tr} \stackrel{\circ}{\mathbf{B}}=-\sigma_{J}<0$. Thus, imposing the condition $c_{6} c_{7}>0$ leads to $\stackrel{\circ}{\xi}^{2}>-\left(\stackrel{\circ}{B}^{2}+\stackrel{\circ}{E}^{2}+3 \mathcal{E}\right) / 26$. However, in this case the relations $\mathcal{C}(\mathcal{A}, T, \Omega)$ and $\mathcal{C}(\mathcal{A}, Q)$ hold. These cannot be satisfied for ordinary matter density (i.e. such that $\rho>0$ ) and the given equation of state.

The case $\mathcal{A}=0, T=0, \xi=0(\mathcal{A} \mathbf{T} \xi \boldsymbol{\Omega})$ : the assumptions $\mathcal{A}=0, \xi=0$ and $\Omega=0$ lead using equation $59 \mathrm{~g}$ to $\mathcal{B}=0$. From equation 60a one obtains the conditions $\mathcal{C}(\mathcal{A}, T, \Omega)$, and $\dot{\Phi}=\dot{B}=0$. Thus, we analyse the system $(\mathcal{A} \mathbf{T} \xi \boldsymbol{\Omega} \mathcal{B})$. There is a zero eigenvalue with multiplicity 3 . In addition, one has $\lambda_{1}=-\sigma_{J}<0$ and $\lambda_{ \pm}=\frac{1}{4}\left(-\left(3+v_{s}\right) 3 \stackrel{\circ}{\Sigma} \pm \sqrt{8\left(1+v_{s}\right)^{2} \stackrel{\circ}{\rho}+\left(v_{s}-1\right)^{2}(3 \stackrel{\circ}{\Sigma})^{2}}\right)$, the condition $\lambda_{ \pm}<0$ is satisfied for

$$
\stackrel{\circ}{\Sigma}>2\left(1+v_{s}\right) \sqrt{\stackrel{\circ}{\rho} /\left(3\left(v_{s}+5+2 \sqrt{3}\right)\left(v_{s}+5-2 \sqrt{3}\right)\right)} .
$$


The case $\mathcal{A}=0, Q=0, \xi=0(\mathcal{A} \mathbf{Q} \boldsymbol{\Omega})$ : this case reduces to $(\mathcal{A} \mathbf{Q} \xi \boldsymbol{\Omega} \mathcal{B})$ with $\mathcal{C}(\mathcal{A}, Q)$. From the associated rank 6 matrix trace we infer that the system is certainly unstable if $\Sigma^{\circ} \geq \Sigma_{s} \equiv \sigma_{J} / 3\left(6+v_{s}\right)$. On the other hand, if the system is stable, then the following conditions must be verified: (a) $\stackrel{\circ}{\mathcal{E}}>\stackrel{\circ}{\mathcal{E}}_{p}$ with $\stackrel{\circ}{T}>-3 \Sigma_{s}$, or $(\mathrm{b}) \stackrel{\circ}{\mathcal{E}} \leq \stackrel{\circ}{\mathcal{E}_{p}}$ and $\stackrel{\circ}{T}>\stackrel{\circ}{T}_{p}$ where we introduced the following notation

$$
\begin{aligned}
\dot{\mathcal{E}}_{p} & \equiv \frac{4\left(6+v_{s}\right)^{2}\left(2+3 v_{s}\left(2+v_{s}\right)\right) \stackrel{\circ}{\rho}+3\left(61+4 v_{s}\left(6+v_{s}\right)\right) \sigma_{J}^{2}}{6\left(6+v_{s}\right)^{2}} \\
\stackrel{\circ}{T}_{p} & \equiv \frac{-6\left(5+v_{s}\right) \sigma_{J}+\sqrt{6} \sqrt{\left(59+20 v_{s}\right)\left(2\left(2+3 v_{s}\left(2+v_{s}\right)\right) \stackrel{\circ}{(5 \mathcal{E})+6\left(5+v_{s}\right)^{2} \sigma_{J}^{2}}\right.}}{177+60 v_{s}} .
\end{aligned}
$$

This condition correspond to the requirements $c_{4}>0$ and $\operatorname{Tr} \stackrel{\circ}{\mathbf{B}}<0$ arising, in turn, from $c_{5}=-\operatorname{Tr} \stackrel{\circ}{\mathbf{B}}>0$ and $c_{4} c_{5}>0$. It can be also written as: $\stackrel{o}{\Sigma}<\Sigma_{s}$ and $(\stackrel{o}{E})^{2}+(\stackrel{o}{B})^{2}<-\left[3+v_{s}\left(9+4 v_{s}\right)\right] \stackrel{o}{\rho}+3 \stackrel{o}{\Sigma}\left[3\left(59+20 v_{s}\right) \stackrel{o}{\Sigma}-4\left(5+v_{s}\right) \sigma_{J}\right]$.

The case $\mathcal{A}=0, \Phi=0$ and $\xi=0$ The case $(\mathcal{A} \boldsymbol{\Phi} \xi \boldsymbol{\Omega})$ reduces to $(\mathcal{A} \boldsymbol{\Phi} \xi \boldsymbol{\Omega} \mathcal{B})$. We can say that the rank 6 system is unstable when $\stackrel{\circ}{\Theta} \leq 3\left(5 \Sigma^{\circ}-2 \sigma_{J}\right) / 2\left(14+3 v_{s}\right)$.

The case $\mathcal{A}=0, T=0, Q=0$ and $\xi=0$ In this case one has $(\mathcal{A} \mathbf{T} \mathbf{Q} \xi \boldsymbol{\Omega})$ which reduces to $(\mathcal{A} \mathbf{T} \mathbf{Q} \boldsymbol{\Omega} \mathcal{B})$. This system is characterised by $\dot{\Phi}=0, \dot{B}=0, \dot{\mathcal{E}}=-j$. There are the eigenvalues $\lambda=0$ with multiplicity 4 and $\lambda_{1}=-\sigma_{J}<0$. Nevertheless, one has that $\mathcal{C}(\mathcal{A}, T, \Omega)$ and $\mathcal{C}(\mathcal{A}, Q$. These conditions cannot be satisfied by the assumptions $\rho>0$ and the given equation of state.

[1] Z. B. Etienne, Y. T. Liu, \& S. L. Shapiro, Relativistic magnetohydrodynamics in dynamical spacetimes: a new AMR implementation, Phys. Rev. D 82, 084031 (2010).

[2] J. A. Font, Numerical hydrodynamics in general relativity, Living Rev. Rel. 6 (2003).

[3] J. A. Font, Numerical hydrodynamics and magnetohydrodynamics in general relativity, Living Rev. Rel. 11, 7 (2007) .

[4] M. Alcubierre, Introduction to $3+1$ numerical Relativity, Oxford University Press, 2008.

[5] B. Giacommazo \& L. Rezzolla, WhiskeyMHD: a new numerical code for general relativistic MHD, Class. Quantum Grav. 24, S235 (2007).

[6] A. Lichnerowicz, Relativistic hydrodynamics and magnetohydrodynamics, Benjamin, New York, 1967.

[7] L. Anton, O.Zanotti, J.A.Miralles, J. M. Marti, J. M. Ibanez, J. A. Font and J. A. Pons, Numerical $3+1$ general relativistic magnetohydrodynamics: A Local characteristic approach, Astrophys. J. 637, 296 (2006).

[8] D.Radice, L.Rezzolla and F.Galeazzi, High-Order Fully General-Relativistic Hydrodynamics: new Approaches and Tests, Class. Quant. Grav. 31,075012 (2014).

[9] H.Witek, Numerical Relativity in higher-dimensional space-times, Int. J. Mod. Phys. A 28, 1340017 (2013).

[10] D. Pugliese and J.A. Valiente Kroon, On the evolution equations for ideal magnetohydrodynamics in curved spacetime, Gen. Rel. Grav. 44, 2785 (2012).

[11] Y. Choquet-Bruhat, General Relativity and the Einstein equations, Oxford University Press, 2008.

[12] M. Shibata \& Y. Sekiguchi, Magnetohydrodynamics in full general relativity: formulations and tests, Phys. Rev. D 72, 044014 (2005).

[13] T. W. Baumgarte \& S. L. Shapiro, General relativistic magnetohydrodynamics for the numerical construction of dynamical spacetimes, Astrophys. J. 585, 921 (2003).

[14] C. Palenzuela, D. Garrett, L. Lehner, \& S. Liebling, Magnetospheres of black hole systems in force-free plasma, Phys. Rev. D 82, 044045 (2010).

[15] A.M. Anile Relativistic fluids and magneto-fluids: With applications in astrophysics and plasma physics, Cambridge UniversityPress, Cambridge, U.K.; New York, U.S.A., 1989.

[16] M. M. Disconzi, On the well-posedness of relativistic viscous fluids, Nonlinearity 27, 1915 (2014).

[17] E. Horst, Symmetric Plasmas and Their Decay, Commun. Math. Phys. 126, 613-633 (1990).

[18] S. C. Hsu, T. J. Awe, S. Brockington, A. Case, J. T. Cassibry, G. Kagan, S. J. Messer, M. Stanic, X. Tang, D. R. Welch, and F. D. Witherspoon, Spherically Imploding Plasma Liners as a Standoff Driver for Magnetoinertial Fusion, Ieee transactions on plasma science 40,5 (2012).

[19] Tai-Ho Tan and Joseph E. Borovsky, Spherically symmetric high-velocity plasma expansions into background gases, Journal of Plasma Physics 35, 02239 (1986).

[20] P. D. Laskyand A. W. C. Lun Gravitational collapse of spherically symmetric plasmas in Einstein-Maxwell spacetimes . Phys. Rev. D 75, 104010 (2007).

[21] R. L. Viana, R. A. Clemente and S. R. Lopes, Spherically symmetric stationary MHD equilibria with azimuthal rotation, Plasma Phys. Control. Fusion 39, 197 (1997). 
[22] Yan Guo and A. Shadi Tahvildar-Zadeh, Formation of singularities in relativistic fluid dynamics and in spherically symmetric plasma dynamics, Contem. Math., 238, 151-161 (1999).

[23] O. Reula, Hyperbolic methods for Einstein's equations, Living Rev. Rel. 3, 1 (1998).

[24] O. Reula, Exponential decay for small nonlinear perturbations of expanding flat homogeneous cosmologies, Phys. Rev. D 60, 083507 (1999).

[25] C. Clarkson, A Covariant approach for perturbations of rotationally symmetric spacetimes, Phys. Rev. D 76, 104034 (2007).

[26] G. F. R. Ellis \& H. van Elst, Cosmological models: Cargese lectures 1998, NATO Adv. Study Inst. Ser. C. Math. Phys. Sci. 541, 1 (1998).

[27] C. A. Clarkson, M. Marklund, G. Betschart, and P. K. S. Dunsby, The Electromagnetic Signature of Black Hole Ring-Down , Astrophys. J. 613, 492 (2004).

[28] M. Marklund and C. Clarkson, The General relativistic MHD dynamo, Mon. Not. Roy. Astron. Soc. 358 (2005) 892.

[29] G. Betschart, and C. A. Clarkson, Scalar field and electromagnetic perturbations on locally rotationally symmetric spacetimes, Class. Quantum Grav. 21, 5587 (2004).

[30] R. B. Burston, 1+1+2 gravitational perturbations on LRS class II spacetimes: decoupling gravito-electromagnetic tensor harmonic amplitudes, Class. Quantum Grav. 25075004 (2008).

[31] C. A. Clarkson and R. K. Barrett, Covariant perturbations of Schwarzschild black holes, Class. Quantum Grav. 203855 (2003).

[32] H. van Elstyxand, G. F. R. Ellis, The covariant approach to LRS perfect fluid spacetime geometries ,Class. Quantum Grav. 13, 1099 (1996).

[33] R. B. Burston and A. W. C. Lun, 1+1+2 Electromagnetic perturbations on general LRS space-times: Regge-Wheeler and Bardeen-Press equations, Class. Quant. Grav. 25 (2008) 075003.

[34] R. B. Burston, 1+1+2 Electromagnetic perturbations on non-vacuum LRS class II space-times: Decoupling scalar and 2-vector harmonic amplitudes, Class. Quant. Grav. 25075002 (2008).

[35] S. S. Komissarov, Mon. Not. R. Astron. Soc. 368, 993-1000 (2006).

[36] O. Zanotti and D. Pugliese, Von Zeipel's theorem for a magnetized circular flow around a compact object, Gen. Rel. Grav. $47(2015) 4,44$.

[37] Bekenstein, J.D., Oron, E.: New conservation laws in general-relativistic magnetohydrodynamics. Phys. Rev. D 18, 1809171819 (1978). DOI 10.1103/PhysRevD.18.1809

[38] Bekenstein, J.D., Oron, E.: Interior magnetohydrodynamic structure of a rotating relativistic star. Phys. Rev. D 19, 2827172837 (1979). DOI 10.1103/PhysRevD.19.2827

[39] H. Stephani, D. Kramer, M. MacCallum, C. Hoenselaers, E. Herlt ,Exact Solutions of Einstein's Field Equations, Cambridge Monographs on Mathematical Physics, Paperback 2009.

[40] C. Lubbe and J. A. Valiente Kroon, A conformal approach for the analysis of the non-linear stability of pure radiation cosmologies, Annals Phys. 328, 1 (2013).

[41] J.M. Stewart, and M. Walker, Proc. R. Soc. London A 431, 49 (1974).

[42] A. Alho, F. C. Mena, \& J. A. Valiente Kroon, The Einstein-Friedrich-nonlinear scalar field system and the stability of scalar field Cosmologies, In arXiv:1006.3778, (2010).

[43] Q. I. Rahman and G. Schmeisser, Analytic Theory of Polynomials: Critical Points, Zeros and Extremal Properties. London Mathematical Society Monographs, Clarendon Press, 2002.

[44] S. Barnett, A New Formulation of the Theorems of Hurwitz, Routh and Sturm J. Inst. Maths Applies 7240 (1971).

[45] H. O. Kreiss and J. Lorenz, Stability for time-dependent differential equations. Acta Numerica 7, 203 (1998).

[46] H. O. Kreiss, O. E. Ortiz and O. A. Reula, Stability of quasi-linear hyperbolic dissipative systems. Journal of Differential Equations 142, 78 (1998).

[47] H. O. Kreiss, G. B. Nagy, O. E. Ortiz and O. A. Reula, Global existence and exponential decay for hyperbolic dissipative relativistic fluid theories. Journal of Mathematical Physics 38, 5272 (1997).

[48] O. E. Ortiz, Stability of nonconservative hyperbolic systems and relativistic dissipative fluids. Journal of Mathematical Physics 42, 1426 (2001).

[49] We refer to 20, 25, 27, 29, 30, 33, 34, for a general discussion concerning the configurations with a unique symmetry direction, described by the Einstein-Maxwell-Euler system. We mention also the well known solution of toroidal magnetic field widely adopted in the axes-symmetric accretion configurations [35, and [36] for a discussion on the case of a poloidal magnetic field where a metric representation is adapted to the direction of the field, as proved by Bekenstein \& Oron [37, 38. Moreover for a deeper and more general discussion about the MHD configurations in spherical symmetry, see for example the work 17,22

[50] As pointed out in 25] in a locally rotationally symmetric spacetime any background quantities are scalars, implying that the vector and tensor quantities are automatically gauge invariant, under linear perturbations as a consequence of the Stewart-Walker lemma [41.

[51] The non-zero coefficients of the characteristic polynomial are: $c_{2}=\left(6 \dot{\xi}^{2} \Omega\right)^{2}>0, c_{3}=\dot{\circ}^{2}\left(8 \sigma_{J} \stackrel{\circ}{E}^{2}+18 \dot{\mathcal{B}}^{\circ}+9 \sigma_{J} \stackrel{\circ}{\Omega}^{2}\right)$,

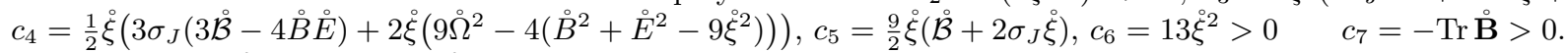

[52] $\operatorname{det} \stackrel{\circ}{\mathbf{B}}=\left(1+v_{s}\right) \dot{\circ}^{2}\left(2 \sigma_{J} \stackrel{\circ}{E}^{2}+3 \dot{B} \dot{\xi}\right)\left(\left(1+3 v_{s}\right) \stackrel{\circ}{\rho}-3 \stackrel{\circ}{Q}^{2}\right)$. 